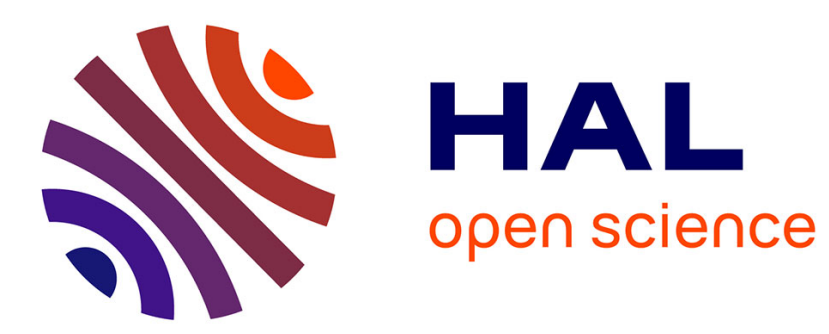

\title{
Singularities of the quad curl problem
}

Serge Nicaise

\section{- To cite this version:}

Serge Nicaise. Singularities of the quad curl problem. Journal of Differential Equations, 2018, 264

(8), pp.5025-5069. 10.1016/j.jde.2017.12.032 . hal-01324641v1

\section{HAL Id: hal-01324641 \\ https://hal.science/hal-01324641v1}

Submitted on 1 Jun 2016 (v1), last revised 14 Dec 2018 (v2)

HAL is a multi-disciplinary open access archive for the deposit and dissemination of scientific research documents, whether they are published or not. The documents may come from teaching and research institutions in France or abroad, or from public or private research centers.
L'archive ouverte pluridisciplinaire HAL, est destinée au dépôt et à la diffusion de documents scientifiques de niveau recherche, publiés ou non, émanant des établissements d'enseignement et de recherche français ou étrangers, des laboratoires publics ou privés. 


\title{
Singularities of the quad curl problem
}

\author{
Serge Nicaise*
}

June 1, 2016

\begin{abstract}
We consider the quad curl problem in smooth and non smooth domains of the space. We first give an augmented variational formulation equivalent to the one from [29] if the datum is divergence free. We describe the singularities of the variational space that correspond to the ones of the Maxwell system with perfectly conducting boundary conditions. The edge and corner singularities of the solution of the corresponding boundary value problem with smooth data are also characterized. We finally obtain some regularity results of the variational solution.
\end{abstract}

AMS (MOS) subject classification 35Q60, 35B65

Key Words Fourth order problem, Maxwell system, singularities

\section{Introduction}

On a bounded domain $\Omega$ of $\mathbb{R}^{3}$, we consider the following system, called the quad curl problem in $[24,29]$

$$
\begin{cases}\operatorname{curl}^{4} \mathbf{u}=\mathbf{f} & \text { in } \Omega, \\ \operatorname{div} \mathbf{u}=0 & \text { in } \Omega, \\ \mathbf{u} \times \mathbf{n}=(\operatorname{curl} \mathbf{u}) \times \mathbf{n}=0 & \text { on } \partial \Omega,\end{cases}
$$

where $\mathbf{f}$ belongs to $\mathbf{H}(\operatorname{div}=0, \Omega)=\{\mathbf{u} \in \mathbf{H}(\operatorname{div}, \Omega): \operatorname{div} \mathbf{u}=0\}$.

This model problem arises in different applications, like in inverse electromagnetic scattering theory [6, 24, 29] or in magnetohydrodynamics [31]. Some numerical methods are proposed in $[29,31,18]$ and some error estimates are proved under some regularity assumptions. The $\mathbf{H}^{3}$ regularity of the weak solution is even assumed in [29, p. 190]. As mentioned in [29], such regularity results are not available in the literature. Hence our goal is to prove such results in the case of smooth and non smooth domains. For that purpose, in the spirit of [7], we first transform the variational formulation from [29] into an augmented one. We show that this augmented problem is well-posed and give its equivalence with the original one when the datum is divergence free. The advantage of this augmented formulation is that its associated boundary value problem is an elliptic system in the Agmon-Douglis-Niremberg sense (but with unsual boundary conditions). The drawback is, as for the variational formulation from [29], that the variational space is a priori not a closed subspace of $H^{2}(\Omega)^{3}$ (except if the domain is smooth or under some very restricted geometrical conditions, see below). Nevertheless in the case of a domain with point singularities or

*Université de Valenciennes et du Hainaut Cambrésis, LAMAV, FR CNRS 2956, Institut des Sciences et Techniques of Valenciennes, F-59313 - Valenciennes Cedex 9 France, Serge.Nicaise@univ-valenciennes.fr 
with corners and edges, we show that the singularities of this variational space are the ones of the Maxwell system with the perfect conductor boundary conditions. According to the results from [7], they are then fully described with the help of the singularities of the Laplace equation with Dirichlet or Neumann boundary conditions.

In the case of smooth domains, even if the boundary conditions of our augmented problem are unsual, they enter in the framework of elliptic systems and therefore, owing to the subjectivity of a trace operator and an appropriated Green formula, we can deduce that the variational solution is smooth if the datum is smooth.

On the contrary, for non-smooth domains regularity results developed for instance in $[19,16$, 8,21 ] cannot be directly applied to our system. Hence we here describe the edge and corner singularities of the augmented problem by adapting the cascade method from [7] (reduction to a lower triangular system). As in that reference, for the two-dimensional and three-dimensional corner singularities, we find three types of singularities. Type 1 are exactly those of the Maxwell system with perfect conductor boundary conditions, while those of type 2 or 3 are fully different from the ones of [7]. The two-dimensional corner singularities are also characterized with the help of the singularities of the Laplace equation with Dirichlet boundary conditions, while in the three-dimensional case, new sets of singular exponents appear that are related to the poles of a holomorphic family of Fredholm operator of index zero. The three-dimensional edge singularities are obtained with the help of two-dimensional singularities of the biharmonic operator with Dirichlet boundary conditions, that are also well-known $[28,16]$ and of the two-dimensional corner singularities.

The characterization of the edge singularities allow us to show that in polyhedral domains the variational solution of the augmented problem is not in $H^{3}(\Omega)^{3}$ in general. But since our problem is not easily localizable, we are not able to show that this variational solution admits a decomposition into a regular part and an explicit singular one. We believe that such a result holds but it requires more investigations. On the contrary, in the case of domains with point singularities, we can apply global regularity results in weighted Sobolev spaces described in section 8.2 of [21] for instance. Such a result combined with our characterization of our variational space allows to obtain a decomposition of the weak solution into a regular part and an explicit singular one. Unfortunately similar global regularity results in weighted Sobolev spaces are not available for fourth order operators in polyhedral domains. This is again a question that merits to be investigated in the future.

The paper is organized as follows: In section 2 we introduce some notations and some function spaces. The augmented variational formulation is introduced in section 3, where its well-posedness and its equivalence with the original problem are analyzed. Some regularity results of the variational space are also presented. The next section 4 is devoted to regularity results in the case of a domain with a smooth boundary. In sections 5 and 6 , we describe the edge and corner singularities; in particular for the three-dimensional corner singularities we introduce a family of operator depending on a complex parameter and prove some useful properties. Finally section 7 concerns regularity results in domains with point singularities.

\section{Some notations and function spaces}

In the whole paper $\Omega$ will be a bounded and simply connected domain of $\mathbb{R}^{3}$ with a connected and Lipschitz boundary. Three cases will retained our attention:

Case 1. the domain has a smooth boundary;

Case 2. the domain has point singularities, i.e., the boundary is smooth, except at a finite 
number of points $c \in \mathcal{C}$, for which $\Omega$ coincides with a three-dimensional cone $\Gamma_{c}$ in a sufficiently small neighborhood of $c$, such points will be called the corners of $\Omega$;

Case 3. the domain has corner points and edges, namely the boundary is piecewise plane (i.e., its boundary is a finite union of polygons). In such a situation, the boundary of $\Omega$ is smooth, except at the corner points and along the edges. Hence we will denote by $\mathcal{C}$ the (finite) set of corners of $\Omega$, and by $\mathcal{E}$ the (finite) set of edges. This means that in a neighborhood of a corner $c, \Omega$ behaves like a three-dimensional cone $\Gamma_{c}$, while near an interior point of an edge $e, \Omega$ behaves like a dihedral cone $C_{e} \times \mathbb{R}$. In this case we also say that $\Omega$ is a polyhedral domain.

For shortness, in the case 1 , we set $\mathcal{C}=\mathcal{E}=\emptyset$, while in the case 2 , we set $\mathcal{E}=\emptyset$.

For a subset $\mathcal{O}$ of $\Omega$ or of the unit sphere and a real number $s, H^{s}(\mathcal{O})$ is the usual Sobolev space defined in $\mathcal{O}$ and for shortness we denote $\mathbf{H}^{s}(\mathcal{O})=H^{s}(\mathcal{O})^{3}$ and $\mathbf{L}^{2}(\mathcal{O})=L^{2}(\mathcal{O})^{3}$. Their norm (resp. semi-norm) will be denoted by $\|\cdot\|_{s, \mathcal{O}}\left(\operatorname{resp} .|\cdot|_{s, \mathcal{O}}\right)$; for $s=0$, we drop the index 0 and for $\mathcal{O}=\Omega$, we also drop the index $\Omega$. As usual $H_{0}^{1}(\Omega)$ is the subspace of $H^{1}(\Omega)$ with a zero trace on the boundary. We further recall that

$$
\begin{aligned}
\mathbf{H}(\operatorname{div}, \Omega) & =\left\{\mathbf{u} \in \mathbf{L}^{2}(\Omega): \operatorname{div} \mathbf{u} \in L^{2}(\Omega)\right\} \\
\mathbf{H}(\operatorname{curl}, \Omega) & =\left\{\mathbf{u} \in \mathbf{L}^{2}(\Omega): \operatorname{curl} \mathbf{u} \in \mathbf{L}^{2}(\Omega)\right\} \\
\mathbf{H}_{0}(\operatorname{curl}, \Omega) & =\{\mathbf{u} \in \mathbf{H}(\operatorname{curl}, \Omega): \mathbf{u} \times \mathbf{n}=0 \text { on } \partial \Omega\}, \\
X_{N}(\Omega) & =\mathbf{H}_{0}(\operatorname{curl}, \Omega) \cap \mathbf{H}(\operatorname{div}, \Omega),
\end{aligned}
$$

are Hilbert spaces with their natural norm. For further purposes, we set

$$
\mathbf{H}_{0}^{2}(\operatorname{curl}, \Omega)=\left\{\mathbf{u} \in \mathbf{H}_{0}(\operatorname{curl}, \Omega): \operatorname{curl} \mathbf{u} \in \mathbf{H}_{0}(\operatorname{curl}, \Omega)\right\},
$$

that is again a Hilbert space with the norm

$$
\|\mathbf{u}\|_{2, \operatorname{curl}, \Omega}^{2}:=\|\mathbf{u}\|^{2}+\|\operatorname{curl} \mathbf{u}\|^{2}+\|\operatorname{curl} \operatorname{curl} \mathbf{u}\|^{2} .
$$

Let us denote by $-\Delta_{\text {Dir }}$ the Laplace operator with Dirichlet boundary conditions, defined by

$$
D\left(-\Delta_{\text {Dir }}\right):=\left\{u \in H_{0}^{1}(\Omega): \Delta u \in L^{2}(\Omega)\right\},
$$

and

$$
-\Delta_{\text {Dir }} u=-\Delta u, \forall u \in D\left(-\Delta_{\text {Dir }}\right) .
$$

It is well-known that this operator is a positive self adjoint operator, and that it is also an isomorphism from $D\left(\left(-\Delta_{\text {Dir }}\right)^{s}\right)$ to $D\left(\left(-\Delta_{\text {Dir }}\right)^{s-1}\right)$, for all $s \in \mathbb{R}$. In particular for $s=\frac{1}{2}$, as $D\left(\left(-\Delta_{\text {Dir }}\right)^{\frac{1}{2}}\right)=H_{0}^{1}(\Omega)$ and $D\left(\left(-\Delta_{\text {Dir }}\right)^{-\frac{1}{2}}\right)=H^{-1}(\Omega)$, for any $g \in H^{-1}(\Omega), w_{g}=\left(-\Delta_{\text {Dir }}\right)^{-1} g$ belongs to $H_{0}^{1}(\Omega)$ and is the unique solution of

$$
\int_{\Omega} \nabla w_{g} \cdot \nabla \bar{y}=\langle g, y\rangle, \forall y \in H_{0}^{1}(\Omega) .
$$

For further uses, let us recall the following Green formulas

$$
\begin{array}{r}
\int_{\Omega}(\nabla u \cdot \mathbf{v}+u \operatorname{div} \mathbf{v})=0, \forall u \in H_{0}^{1}(\Omega), \mathbf{v} \in H(\operatorname{div}, \Omega), \\
\int_{\Omega}(\operatorname{curl} \mathbf{u} \cdot \mathbf{v}-\mathbf{u} \cdot \operatorname{curl} \mathbf{v})=0, \forall \mathbf{u} \in H_{0}(\operatorname{curl}, \Omega), \mathbf{v} \in H(\operatorname{curl}, \Omega),
\end{array}
$$


see formula (I.2.17) of [10] for the first one and Remark 3.28 in [23] for the second one.

By Leibniz's rule, one can show that for any (smooth enough) vector fields a and scalar field $q$, we have

$$
\begin{array}{r}
\operatorname{curl}(q \mathbf{a})=q \operatorname{curl} \mathbf{a}+\nabla q \times \mathbf{a}, \\
\nabla(\mathbf{a} \cdot \mathbf{x})=\left(r \partial_{r}+1\right) \mathbf{a}+\mathbf{x} \times \operatorname{curl} \mathbf{a}, \\
\operatorname{curl}(\mathbf{a} \times \mathbf{x})=\left(r \partial_{r}+2\right) \mathbf{a}-\mathbf{x} \operatorname{div} \mathbf{a}, \\
\operatorname{curl}(q \mathbf{x})=\nabla q \times \mathbf{x}, \\
\operatorname{curl} \operatorname{curl}(q \mathbf{x})=\left(r \partial_{r}+2\right) \nabla q-\mathbf{x} \Delta q .
\end{array}
$$

Note that the identity (2.7) is a direct consequence of (2.5) and (2.6).

Finally in the whole paper, the notation $A \lesssim B$ is used for the estimate $A \leq C B$, where $C$ is a generic constant that does not depend on $A$ and $B$. The notation $A \sim B$ means that both $A \lesssim B$ and $B \lesssim A$ hold.

\section{Augmented variational formulation}

The variational formulation of (1.1) from [29] consists in looking for $\mathbf{u} \in V_{0}:=\left\{\mathbf{u} \in \mathbf{H}_{0}^{2}(\operatorname{curl}, \Omega)\right.$ : $\operatorname{div} \mathbf{u}=0\}$ solution of

$$
\int_{\Omega} \operatorname{curl}^{2} \mathbf{u} \cdot \operatorname{curl}^{2} \overline{\mathbf{v}}=\int_{\Omega} \mathbf{f} \cdot \overline{\mathbf{v}}, \forall \mathbf{v} \in V_{0},
$$

and is well posed since this left-hand side is a coercive form on $V_{0}$ (see Theorem 1 of [29]).

In order to relax the divergence free constraint in $V_{0}$, we propose the following alternative formulation. Introduce the space

$$
V:=\left\{\mathbf{u} \in \mathbf{H}_{0}^{2}(\operatorname{curl}, \Omega): \operatorname{div} \mathbf{u} \in H_{0}^{1}(\Omega)\right\}
$$

that is a Hilbert space with the norm

$$
\|\mathbf{u}\|_{V}^{2}=\|\mathbf{u}\|_{2, \operatorname{curl}, \Omega}^{2}+|\operatorname{div} \mathbf{u}|_{1}^{2} .
$$

The augmented variational formulation of (1.1) consists in looking for $\mathbf{u} \in V$ solution of

$$
a(\mathbf{u}, \mathbf{v})=\int_{\Omega} \mathbf{f} \cdot \overline{\mathbf{v}}, \forall \mathbf{v} \in V
$$

where

$$
a(\mathbf{u}, \mathbf{v})=\int_{\Omega}(\operatorname{curl} \operatorname{curl} \mathbf{u}-\nabla \operatorname{div} \mathbf{u}) \cdot(\operatorname{curl} \operatorname{curl} \overline{\mathbf{v}}-\nabla \operatorname{div} \overline{\mathbf{v}}), \forall \mathbf{u}, \mathbf{v} \in V
$$

Let us notice that $a$ admits the equivalent expression

$$
a(\mathbf{u}, \mathbf{v})=\int_{\Omega} \Delta \mathbf{u} \cdot \Delta \overline{\mathbf{v}}
$$

since for $\mathbf{u} \in V$, we have curl curl $\mathbf{u}-\nabla \operatorname{div} \mathbf{u}=-\Delta \mathbf{u}$ in $\mathcal{D}^{\prime}(\Omega)^{3}$ (and hence $\Delta \mathbf{u}$ belongs to $\mathbf{L}^{2}(\Omega$ ) as the sum of two elements of $\mathbf{L}^{2}(\Omega)$ ).

Before any further considerations, let us show that the space $V$ and the variational problem (3.4) are invariant by translation and rotation. 
Theorem 3.1 Let $R$ be an orthogonal matrix with determinant 1 and let $c \in \mathbb{R}^{3}$ be fixed. Let $F$ be the transformation from $\mathbb{R}^{3}$ into itself defined by $F(\hat{\mathbf{x}})=R \hat{\mathbf{x}}+\mathbf{c}$, that corresponds to a translation of $\mathbf{c}$ and a rotation of $R$. Let $\hat{\Omega}=F^{-1}(\Omega)$. Then for any $\mathbf{u} \in V$, the vector field $\hat{\mathbf{u}}$ defined by the covariant transformation

$$
\hat{\mathbf{u}}=R^{-1}(\mathbf{u} \circ F)
$$

belongs to

$$
\hat{V}=\left\{\mathbf{v} \in \mathbf{H}_{0}^{2}(\operatorname{curl}, \hat{\Omega}): \operatorname{div} \mathbf{v} \in H_{0}^{1}(\hat{\Omega})\right\} .
$$

Furthermore if $\mathbf{u} \in V$ is a solution of (3.3), then $\hat{\mathbf{u}}$ given by (3.5) is solution of

$$
\hat{a}(\hat{\mathbf{u}}, \hat{\mathbf{v}})=\int_{\hat{\Omega}} R^{-1}(\mathbf{f} \circ F) \cdot \overline{\hat{\mathbf{v}}}, \forall \hat{\mathbf{v}} \in \hat{V},
$$

where $\hat{a}$ is defined as a with $\hat{\Omega}$ instead of $\Omega$.

Proof. It turns out that for a rotation matrix $R$ the covariant and contravariant transformations coincide, hence by the chain rule, see for instance [4, p. 59-63], we have

$$
\begin{array}{r}
\operatorname{div} \mathbf{u}=\operatorname{div} \hat{\mathbf{u}}, \operatorname{curl} \mathbf{u}=R \operatorname{curl} \hat{\mathbf{u}}, \\
\nabla \operatorname{div} \mathbf{u}=R \hat{\nabla} \hat{\operatorname{div}} \hat{\mathbf{u}}, \operatorname{curl} \operatorname{curl} \mathbf{u}=R \operatorname{curl} \operatorname{curl} \hat{\mathbf{u}} .
\end{array}
$$

The two assertions follow from these identities and the fact that $\mathbf{u} \times \mathbf{n}=\mathbf{0}$ on $\partial \Omega$ if and only if $\hat{\mathbf{u}} \times \hat{\mathbf{n}}=\mathbf{0}$ on $\partial \hat{\Omega}$.

We now show that problem (3.3) is well posed and that it is equivalent to (3.1) is $\mathbf{f}$ is divergence free.

Theorem 3.2 For any $\mathbf{f} \in \mathbf{L}^{2}(\Omega)$, there exists a unique solution $\mathbf{u} \in V$ of (3.3). The divergence of this solution is the unique solution in $H_{0}^{1}(\Omega)$ of

$$
\int_{\Omega} \nabla \operatorname{div} \mathbf{u} \cdot \nabla \bar{g}=\int_{\Omega}\left(-\Delta_{\text {Dir }}\right)^{-1} \operatorname{div} \mathbf{f} \bar{g}, \forall g \in H_{0}^{1}(\Omega) .
$$

Hence in particular, it satisfies

$$
-\Delta \operatorname{div} \mathbf{u}=\left(-\Delta_{\text {Dir }}\right)^{-1} \operatorname{div} \mathbf{f} \in H_{0}^{1}(\Omega) .
$$

Consequently if $\mathbf{f}$ is divergence free, then $\mathbf{u}$ is divergence free and is also the unique solution of (3.1).

Proof. From (3.4), for any $\mathbf{u} \in V$, we have

$$
\begin{aligned}
a(\mathbf{u}, \mathbf{u}) & =\int_{\Omega}|\operatorname{curl} \operatorname{curl} \mathbf{u}-\nabla \operatorname{div} \mathbf{u}|^{2} \\
& =\int_{\Omega}|\operatorname{curl} \operatorname{curl} \mathbf{u}|^{2}+|\nabla \operatorname{div} \mathbf{u}|^{2}-2 \Re \int_{\Omega} \operatorname{curl} \operatorname{curl} \mathbf{u} \cdot \nabla \operatorname{div} \overline{\mathbf{u}} .
\end{aligned}
$$

As curl curl $\mathbf{u}$ belongs to $H(\operatorname{div}, \Omega)$ and $\operatorname{div} \mathbf{u}$ is in $H_{0}^{1}(\Omega)$, by Green's formula (2.1), we find that

$$
\int_{\Omega} \operatorname{curl} \operatorname{curl} \mathbf{u} \cdot \nabla \operatorname{div} \overline{\mathbf{u}}=0
$$


This shows the identity

$$
a(\mathbf{u}, \mathbf{u})=\int_{\Omega}\left(|\operatorname{curl} \operatorname{curl} \mathbf{u}|^{2}+|\nabla \operatorname{div} \mathbf{u}|^{2}\right), \forall \mathbf{u} \in V .
$$

Hence by Poincaré's inequality in $H_{0}^{1}(\Omega)$, we find that

$$
a(\mathbf{u}, \mathbf{u}) \gtrsim \int_{\Omega}\left(|\operatorname{curl} \operatorname{curl} \mathbf{u}|^{2}+|\nabla \operatorname{div} \mathbf{u}|^{2}+|\operatorname{div} \mathbf{u}|^{2}\right), \forall \mathbf{u} \in V .
$$

From the Friedrichs inequality in $X_{N}(\Omega)$ (see Corollary 3.19 in [1]), we get

$$
\int_{\Omega}|\operatorname{curl} \operatorname{curl} \mathbf{u}|^{2} \gtrsim \int_{\Omega}\left(|\operatorname{curl} \operatorname{curl} \mathbf{u}|^{2}+|\operatorname{curl} \mathbf{u}|^{2}\right),
$$

as curl $\mathbf{u}$ belongs to $X_{N}(\Omega)$. As $\mathbf{u}$ itself belongs to $X_{N}(\Omega)$, applying again Friedrichs inequality in $X_{N}(\Omega)$, we find that

$$
\int_{\Omega}\left(|\operatorname{curl} \mathbf{u}|^{2}+|\operatorname{div} \mathbf{u}|^{2}\right) \gtrsim \int_{\Omega}\left(|\operatorname{curl} \mathbf{u}|^{2}+|\operatorname{div} \mathbf{u}|^{2}+|\mathbf{u}|^{2}\right) .
$$

The two previous estimates in the estimate (3.8) lead to the coerciveness of $a$ on $V$, namely

$$
a(\mathbf{u}, \mathbf{u}) \gtrsim\|\mathbf{u}\|_{V}^{2} .
$$

By Lax-Milgram lemma, we deduce the well-posedness of (3.3).

For the second assertion, it suffices to take in (3.3) a test function $\mathbf{v}=\nabla w_{g}$, with an arbitrary $g \in H_{0}^{1}(\Omega)$, where $w_{g}=\left(-\Delta_{\text {Dir }}\right)^{-1} g$. Note that $w_{g}$ also satisfies

$$
-\Delta w_{g}=g
$$

in the distributional sense and hence $\operatorname{div} \mathbf{v}=-g \in H_{0}^{1}(\Omega)$. Then we get

$$
\int_{\Omega}(\operatorname{curl} \operatorname{curl} \mathbf{u}-\nabla \operatorname{div} \mathbf{u}) \cdot \nabla \bar{g}=\int_{\Omega} \mathbf{f} \cdot \nabla \bar{w}_{g} .
$$

As curl curl $\mathbf{u}$ belongs to $H(\operatorname{div}, \Omega)$ and is divergence free, by Green's formula (2.1) in the left-hand side, we find that

$$
\int_{\Omega} \nabla \operatorname{div} \mathbf{u} \cdot \nabla \bar{g}=\left\langle\operatorname{div} \mathbf{f} ; w_{g}\right\rangle_{H^{-1}(\Omega)-H_{0}^{1}(\Omega)},
$$

reminding that $\mathbf{f}$ is assumed to be in $\mathbf{L}^{2}(\Omega)$. Since $L^{2}(\Omega)$ is the pivot space between $H^{-1}(\Omega)$ and $H_{0}^{1}(\Omega)$, we have

$$
\left\langle\operatorname{div} \mathbf{f} ; w_{g}\right\rangle_{H^{-1}(\Omega)-H_{0}^{1}(\Omega)}=\left\langle\operatorname{div} \mathbf{f} ;\left(-\Delta_{\text {Dir }}\right)^{-1} g\right\rangle_{H^{-1}(\Omega)-H_{0}^{1}(\Omega)}=\int_{\Omega}\left(-\Delta_{\text {Dir }}\right)^{-1}(\operatorname{div} \mathbf{f}) \bar{g} .
$$

The two previous identities lead to (3.6). As this identity implies that

$$
-\Delta \operatorname{div} u=\left(-\Delta_{\text {Dir }}\right)^{-1} \operatorname{div} \mathbf{f}
$$

in the distributional sense, we directly obtain (3.7) (since $\operatorname{div} \mathbf{f} \in H^{-1}(\Omega)$ ).

Finally if $\mathbf{f}$ is divergence free, the right-hand side of (3.6) is zero and consequenctly div $\mathbf{u}=0$ and therefore $\mathbf{u} \in V_{0}$. Reducing (3.3) to test functions in $V_{0}$ leads to the fact that $\mathbf{u}$ is also solution of $(3.1)$. 
Corollary 3.3 For any $\mathbf{f} \in \mathbf{L}^{2}(\Omega)$, the unique solution $\mathbf{u} \in V$ of (3.3) satisfies the boundary value problem

$$
\begin{cases}\Delta^{2} \mathbf{u}=\mathbf{f} & \text { in } \Omega, \\ \operatorname{div} \mathbf{u}=\Delta \operatorname{div} \mathbf{u}=0 & \text { on } \partial \Omega, \\ \mathbf{u} \times \mathbf{n}=(\operatorname{curl} \mathbf{u}) \times \mathbf{n}=0 & \text { on } \partial \Omega,\end{cases}
$$

Proof. By taking test functions $\mathbf{v} \in \mathcal{D}(\Omega)^{3}$ in (3.3), we directly find that

$$
\Delta^{2} \mathbf{u}=\mathbf{f} \text { in } \mathcal{D}^{\prime}(\Omega)^{3} .
$$

The boundary conditions

$$
\begin{aligned}
\operatorname{div} \mathbf{u}=0 & \text { on } \partial \Omega, \\
\mathbf{u} \times \mathbf{n}=(\operatorname{curl} \mathbf{u}) \times \mathbf{n}=0 & \text { on } \partial \Omega,
\end{aligned}
$$

come from the fact that $\mathbf{u}$ is in $V$. Finally the boundary condition

$$
\Delta \operatorname{div} \mathbf{u}=0 \text { on } \partial \Omega .
$$

follows from the property (3.7).

It is easy to check that the system (3.9) is an elliptic system in the Agmon-Douglis-Niremberg sense, but its variational formulation is, a priori, not based on a closed subspace of $\mathbf{H}^{2}(\Omega)$. Nevertheless, let us show that its variational space $V$ (and hence $V_{0}$ ) is indeed a subspace of $\mathbf{H}^{2}(\Omega)$ if the boundary of $\Omega$ is smooth and admits a decomposition into regular fields in $\mathbf{H}^{2}(\Omega)$ and singular ones in the two other cases.

Lemma 3.4 If the boundary of $\Omega$ is smooth, then $V$ is a closed subspace of $\mathbf{H}^{2}(\Omega)$. In the case 2 or 3, with the notations of sections 5 and 6 below (see also [7, \$4]), assume that

$$
\left\{\begin{array}{l}
\forall c \in \mathcal{C}, \frac{3}{2} \notin \Lambda_{D i r}\left(\Gamma_{c}\right) \text { and } \frac{1}{2} \notin \Lambda_{N e u}\left(\Gamma_{c}\right), \\
\forall e \in \mathcal{E}, \omega_{e} \notin\left\{\frac{\pi}{2}, \frac{3 \pi}{2}\right\} .
\end{array}\right.
$$

Then any $\mathbf{u} \in V$ admits the decomposition

$$
\mathbf{u}=\mathbf{u}_{\mathrm{reg}}+\mathbf{u}_{\text {sing }},
$$

where $\mathbf{u}_{\text {reg }} \in \mathbf{H}^{2}(\Omega)$ while $\mathbf{u}_{\text {sing }} \in V \backslash \mathbf{H}^{2}(\Omega)$ is a sum of corner and edge singularities.

Proof. Fix $\mathbf{u} \in V$ and $\mathbf{v} \in X_{N}(\Omega)$. As div u belongs to $H_{0}^{1}(\Omega)$ and $\mathbf{v}$ is in $H(\operatorname{div}, \Omega)$, Green's formula (2.1) yields

$$
\int_{\Omega} \operatorname{div} \mathbf{u} \operatorname{div} \overline{\mathbf{v}}=-\int_{\Omega} \nabla \operatorname{div} \mathbf{u} \cdot \overline{\mathbf{v}}
$$

Similarly as curl $\mathbf{u}$ is in $H_{0}(\operatorname{curl}, \Omega)$ and $\mathbf{v}$ is in $H(\operatorname{curl}, \Omega)$, Green's formula (2.2) leads to

$$
\int_{\Omega} \operatorname{curl} \mathbf{u} \cdot \operatorname{curl} \overline{\mathbf{v}}=\int_{\Omega} \operatorname{curl}^{2} \mathbf{u} \cdot \overline{\mathbf{v}} \text {. }
$$

Taking the sum of these two identities, we deduce that $\mathbf{u} \in V$ satisfies

$$
\int_{\Omega}(\operatorname{curl} \mathbf{u} \cdot \operatorname{curl} \overline{\mathbf{v}}+\operatorname{div} \mathbf{u} \operatorname{div} \overline{\mathbf{v}})=\int_{\Omega}\left(\operatorname{curl}^{2} \mathbf{u}-\nabla \operatorname{div} \mathbf{u}\right) \cdot \overline{\mathbf{v}}, \forall \mathbf{v} \in X_{N}(\Omega) .
$$

Therefore we can see $\mathbf{u} \in V$ as the unique solution in $X_{N}(\Omega)$ of (3.12). $\operatorname{As} \operatorname{curl}^{2} \mathbf{u}-\nabla \operatorname{div} \mathbf{u}$ belongs to $\mathbf{L}^{2}(\Omega)$, we can apply Theorem 4.7 of [7] to obtain a decomposition of $\mathbf{u}$ in a regular field

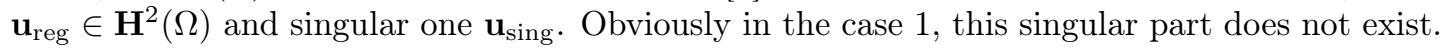


Remark 3.5 If the assumption (3.10) does not hold, then a similar decomposition holds but with $\mathbf{u}_{\mathrm{reg}} \in \mathbf{H}^{2-\varepsilon}(\Omega)$, for $\varepsilon>0$ small enough.

A direct consequence of the previous result is that in the case 2 or $3, V$ is embedded into $\mathbf{H}^{2}(\Omega)$ if and only if the singular part is zero, and therefore if and only if the set of corner (resp. edge) singular exponent $\Lambda_{N, 1}\left(\Gamma_{c}\right)$ (resp. $\Lambda_{N, 1}\left(W_{e}\right)$ ) of problem (3.12) described in [7, §4] is empty. But before stating such a result, let us show that if no edge singular exponents appear, then the contribution of $\Lambda_{N e u}\left(\Gamma_{c}\right)$ in the corner singular part dissappears.

Lemma 3.6 In the case 2 or 3, with the notations of sections 5 and 6 below, assume that

$$
\left\{\begin{array}{l}
\forall c \in \mathcal{C}, \frac{3}{2} \notin \Lambda_{D i r}\left(\Gamma_{c}\right) \text { and } \frac{1}{2} \notin \Lambda_{N e u}\left(\Gamma_{c}\right), \\
\forall e \in \mathcal{E}, \omega_{e}<\frac{\pi}{2} .
\end{array}\right.
$$

Then $\mathbf{u} \in V$ admits the decomposition (3.11) where $\mathbf{u}_{\mathrm{reg}} \in \mathbf{H}^{2}(\Omega)$,

$$
\mathbf{u}_{\text {sing }}=\sum_{c \in \mathcal{C}} \eta_{c} \sum_{\lambda \in\left(-\frac{3}{2}, \frac{1}{2}\right): \lambda+1 \in \Lambda_{\text {Dir }}\left(\Gamma_{c}\right)} \sum_{p} d_{c, \lambda, p} \nabla u_{\text {Dir }}^{\lambda+1, p}
$$

$\eta_{c}$ being a radial cut-off function equal to 1 near $c$ and zero in a neighbourhood of the other corner points of $\Omega$, and for $\lambda \in\left(-\frac{3}{2}, \frac{1}{2}\right)$ such that $\lambda+1 \in \Lambda_{\operatorname{Dir}}\left(\Gamma_{c}\right), d_{c, \lambda, p} \in \mathbb{C}$ and

$$
\left\|\mathbf{u}_{\mathrm{reg}}\right\|_{2, \Omega}+\sum_{c \in \mathcal{C}} \sum_{\lambda \in\left(-\frac{3}{2}, \frac{1}{2}\right): \lambda+1 \in \Lambda_{\operatorname{Dir}}\left(\Gamma_{c}\right)} \sum_{p}\left|d_{c, \lambda, p}\right| \lesssim\|\mathbf{u}\|_{V} .
$$

Proof. Using Lemmas 4.3 and 4.6 of [7] and since $\omega_{e}<\frac{\pi}{2}$, for all $e \in \mathcal{E}$, in the decomposition (3.11), only corner singularities contribute to $\mathbf{u}_{\text {sing }}$, namely (again with the notations from section $6)$

$$
\begin{aligned}
\mathbf{u}_{\text {sing }} & =\sum_{c \in \mathcal{C}} \eta_{c} \sum_{\lambda \in\left(-\frac{3}{2}, \frac{1}{2}\right): \lambda+1 \in \Lambda_{\operatorname{Dir}}\left(\Gamma_{c}\right)} \sum_{p} d_{c, \lambda, p}^{(1)} \nabla u_{\mathrm{Dir}}^{\lambda+1, p} \\
& +\sum_{c \in \mathcal{C}} \eta_{c} \sum_{\lambda \in\left(-\frac{1}{2}, \frac{1}{2}\right) \cap \Lambda_{\mathrm{Neu}}\left(\Gamma_{c}\right)} \sum_{p} d_{c, \lambda, p}^{(2)}\left(\nabla u_{\mathrm{Neu}}^{\lambda, p} \times \mathbf{x}_{c}\right),
\end{aligned}
$$

where $\mathbf{x}_{c}$ is the cartesian coordinates centred at $c$, for $\lambda \in\left(-\frac{3}{2}, \frac{1}{2}\right)$ such that $\lambda+1 \in \Lambda_{\text {Dir }}\left(\Gamma_{c}\right)$, $d_{c, \lambda, p}^{(1)} \in \mathbb{C}$ and for $\lambda \in\left(-\frac{1}{2}, \frac{1}{2}\right) \cap \Lambda_{\mathrm{Neu}}\left(\Gamma_{c}\right), d_{c, \lambda, p}^{(2)} \in \mathbb{C}$.

But the essential boundary condition curl $\mathbf{u}=\mathbf{0}$ on $\partial \Omega$ implies that

$$
\operatorname{curl} \mathbf{u}_{\text {sing }} \in \mathbf{H}^{\frac{1}{2}}(\partial \Omega) .
$$

From the previous splitting of $\mathbf{u}_{\text {sing }}$, we have

$$
\begin{aligned}
\operatorname{curl} \mathbf{u}_{\text {sing }} & =\sum_{c \in \mathcal{C}} \sum_{\lambda \in\left(-\frac{3}{2}, \frac{1}{2}\right): \lambda+1 \in \Lambda_{\mathrm{Dir}}\left(\Gamma_{c}\right)} \sum_{p} d_{c, \lambda, p}^{(1)} \operatorname{curl}\left(\eta_{c} \nabla u_{\mathrm{Dir}}^{\lambda+1, p}\right) \\
& +\sum_{c \in \mathcal{C}} \sum_{\lambda \in\left(-\frac{1}{2}, \frac{1}{2}\right): \lambda \in \Lambda_{\mathrm{Neu}}\left(\Gamma_{c}\right)} \sum_{p} d_{c, \lambda, p}^{(2)} \operatorname{curl}\left(\eta_{c} \nabla u_{\text {Neu }}^{\lambda, p} \times \mathbf{x}_{c}\right) .
\end{aligned}
$$


By (2.3) and (2.5), we have

$$
\begin{aligned}
\operatorname{curl}\left(\eta_{c} \nabla u_{\text {Dir }}^{\lambda+1, p}\right) & =\nabla \eta_{c} \times \nabla u_{\text {Dir }}^{\lambda+1, p}, \\
\operatorname{curl}\left(\eta_{c} \nabla u_{\text {Neu }}^{\lambda, p} \times \mathbf{x}_{c}\right) & =\eta_{c}(\lambda+1) \nabla u_{\text {Neu }}^{\lambda, p}+\nabla \eta_{c} \times\left(\nabla u_{\text {Neu }}^{\lambda, p} \times \mathbf{x}_{c}\right) .
\end{aligned}
$$

As $u_{\text {Dir }}^{\lambda+1, p}$ is in $H^{2}$ far from $c$, we deduce that $\nabla \eta_{c} \times \nabla u_{\text {Dir }}^{\lambda+1, p}$ belongs to $\mathbf{H}^{\frac{1}{2}}(\partial \Omega)$. For the same reason $\nabla \eta_{c} \times\left(\nabla u_{\mathrm{Neu}}^{\lambda, p} \times \mathbf{x}_{c}\right)$ belongs to $\mathbf{H}^{\frac{1}{2}}(\partial \Omega)$. Hence the condition (3.17) implies that

$$
\sum_{c \in \mathcal{C}} \sum_{\lambda \in\left(-\frac{1}{2}, \frac{1}{2}\right): \lambda \in \Lambda_{\mathrm{Neu}}\left(\Gamma_{c}\right)} \eta_{c} d_{c, \lambda, p}^{(2)}(\lambda+1) \nabla u_{\mathrm{Neu}}^{\lambda, p} \in \mathbf{H}^{\frac{1}{2}}(\partial \Omega) .
$$

Since for each $\lambda \in\left(-\frac{1}{2}, \frac{1}{2}\right) \cap \Lambda_{\mathrm{Neu}}\left(\Gamma_{c}\right), \nabla u_{\text {Neu }}^{\lambda, p}$ belongs to $\mathbf{H}^{s}(\partial \Omega)$ if and only if $s<\lambda$, we deduce $d_{c, \lambda, p}^{(2)}=0$ for all $\lambda \in\left(-\frac{1}{2}, \frac{1}{2}\right) \cap \Lambda_{\mathrm{Neu}}\left(\Gamma_{c}\right)$, all $p$, and all $c$. This means that (3.16) reduces to (3.14) with $d_{c, \lambda, p}=d_{c, \lambda, p}^{(1)}$.

The estimate (3.15) is relatively standard and is based on the open mapping theorem. For completeness let us give its proof. Introduce the space

$$
W=\left\{\left(u_{\mathrm{reg}}, d_{c, \lambda, p}\right) \in \mathbf{H}^{2}(\Omega) \times \mathbb{C}^{N}: u_{\mathrm{reg}}+\sum_{c \in \mathcal{C}} \eta_{c} \sum_{\lambda \in\left(-\frac{3}{2}, \frac{1}{2}\right): \lambda+1 \in \Lambda_{\mathrm{Dir}}\left(\Gamma_{c}\right)} \sum_{p} d_{c, \lambda, p} \nabla u_{\mathrm{Dir}}^{\lambda+1, p} \in V\right\},
$$

where $N$ is the cardinal of the set of triples $(c, \lambda, p)$ satisfying the above constraints. This space is a Banach space with the norm

$$
\left\|\left(u_{\mathrm{reg}}, d_{c, \lambda, p}\right)\right\|_{W}=\left\|u_{\mathrm{reg}}\right\|_{2, \Omega}+\sum_{c \in \mathcal{C}} \sum_{\lambda \in\left(-\frac{3}{2}, \frac{1}{2}\right): \lambda+1 \in \Lambda_{\operatorname{Dir}}\left(\Gamma_{c}\right)} \sum_{p}\left|d_{c, \lambda, p}\right|
$$

because

$$
\left\|u_{\mathrm{reg}}+\sum_{c \in \mathcal{C}} \eta_{c} \sum_{\lambda \in\left(-\frac{3}{2}, \frac{1}{2}\right): \lambda+1 \in \Lambda_{\mathrm{Dir}}\left(\Gamma_{c}\right)} \sum_{p} d_{c, \lambda, p} \nabla u_{\mathrm{Dir}}^{\lambda+1, p}\right\|_{V} \lesssim\left\|\left(u_{\mathrm{reg}}, d_{c, \lambda, p}\right)\right\|_{W} .
$$

Now introduce the continuous mapping

$$
T: W \rightarrow V:\left(u_{\mathrm{reg}}, d_{c, \lambda, p}\right) \rightarrow u_{\mathrm{reg}}+\sum_{c \in \mathcal{C}} \eta_{c} \sum_{\lambda \in\left(-\frac{3}{2}, \frac{1}{2}\right): \lambda+1 \in \Lambda_{\mathrm{Dir}}\left(\Gamma_{c}\right)} \sum_{p} d_{c, \lambda, p} \nabla u_{\mathrm{Dir}}^{\lambda+1, p} .
$$

Our first assertion means that $T$ is surjective. Furthermore, $T$ is injective because the functions $\nabla u_{\text {Dir }}^{\lambda+1, p}$ are linearly independent and do not belong to $\mathbf{H}^{2}(\Omega)$. Hence $T$ is bijective and by the open mapping theorem its inverse is also continuous, which proves (3.15).

This result directly yields the

Corollary 3.7 With the notations of sections 5 and 6 below, assume that

$$
\left\{\begin{array}{l}
\forall c \in \mathcal{C},\left(-\frac{1}{2}, \frac{3}{2}\right] \cap \Lambda_{D i r}\left(\Gamma_{c}\right)=\emptyset \text { and } \frac{1}{2} \notin \Lambda_{N e u}\left(\Gamma_{c}\right), \\
\forall e \in \mathcal{E}, \omega_{e}<\frac{\pi}{2} .
\end{array}\right.
$$

Then $V$ is continuously embedded into $\mathbf{H}^{2}(\Omega)$.

From point (b) of subsection 4.4.2 in [7], the assumption (3.18) holds if $\Omega$ is a convex polyheral domain and $\omega_{e}<\frac{\pi}{2}$ for all edges $e$ (reminding that the convexity of $\Omega$ implies that $\lambda>\frac{1}{2}$ for all $\left.\lambda \in \Lambda_{N e u}\left(\Gamma_{c}\right)\right)$. As a consequence the convexity of $\Omega$ is far from being sufficient to guarantee the $\mathbf{H}^{2}$ regularity of the solution of (3.3) (or (3.1)). Nevertheless, we always get the inclusion $V \subset \mathbf{H}^{\frac{1}{2}+\varepsilon}(\Omega)$, with $\varepsilon>0$ depending on the geometry of $\Omega$. 


\section{Regularity results in the smooth case}

Even if system (3.9) is an elliptic system in the Agmon-Douglis-Niremberg sense, it is a non standard one and, to our best knowledge, the regularity $\mathbf{H}^{4}(\Omega)$ of its weak solution with $\mathbf{f} \in \mathbf{L}^{2}(\Omega)$ is not directly available in the literature. Nevertheless, a priori error estimates are available owing to the general theory of elliptic systems, leading to Fredholm property of the associated operator (see [21, Thm 4.2.4]), hence we are mainly reduced to study the cokernel of the associated operator. This will be made via the subjectivity of the trace mapping from Lemma 4.1 below and the use of an adapted Green formula.

In order to formulate the Fredholm property, we introduce the following operators: for any $\ell \in \mathbb{N}$ and $\mathbf{u} \in \mathbf{H}^{4+\ell}(\Omega)$, if $\gamma$ is the standard trace operator, let us set

$$
B \mathbf{u}=((\gamma \mathbf{u}) \times \mathbf{n}, \gamma(\operatorname{curl} \mathbf{u}) \times \mathbf{n}, \gamma \operatorname{div} \mathbf{u}, \gamma \Delta \operatorname{div} \mathbf{u})^{\top},
$$

that clearly belongs to $R^{\ell}:=\mathbf{H}_{T}^{\frac{7}{2}+\ell}(\partial \Omega) \times \mathbf{H}_{T}^{\frac{5}{2}+\ell}(\partial \Omega) \times H^{\frac{5}{2}+\ell}(\partial \Omega) \times H^{\frac{1}{2}+\ell}(\partial \Omega)$, where

$$
\mathbf{L}_{T}^{2}(\partial \Omega)=\left\{\mathbf{v} \in \mathbf{L}^{2}(\partial \Omega): \mathbf{v} \cdot \mathbf{n}=\mathbf{0}\right\} .
$$

and for any $s>0$,

$$
\mathbf{H}_{T}^{s}(\partial \Omega)=\mathbf{H}^{s}(\partial \Omega) \cap \mathbf{L}_{T}^{2}(\partial \Omega) .
$$

Similarly for any $\mathbf{u} \in \mathbf{H}^{4}(\Omega)$ we denote by

$$
\begin{array}{r}
T \mathbf{u}=(\mathbf{n} \times(\gamma \operatorname{curl} \Delta \mathbf{u}) \times \mathbf{n}, \mathbf{n} \times(\gamma \Delta \mathbf{u}) \times \mathbf{n}, \gamma \Delta \mathbf{u} \cdot \mathbf{n}, \gamma \mathbf{u} \cdot \mathbf{n})^{\top}, \\
D \mathbf{u}=\left(\gamma \mathbf{u}, \gamma \partial_{\mathbf{n}} \mathbf{u}, \gamma \partial_{\mathbf{n}}^{2} \mathbf{u}, \gamma \partial_{\mathbf{n}}^{3} \mathbf{u}\right)^{\top} .
\end{array}
$$

The system $B$ alone is not a "Dirichlet" system (for each component of $\mathbf{u}$ ) since $B \mathbf{u}=\mathbf{0}$ on $\partial \Omega$ does not imply $\mathbf{u} \cdot \mathbf{n}=0$ on $\partial \Omega$, but the full system $(B, T)$ is a "Dirichlet" system as the next Lemma shows. For shortness let us set

$$
S^{0}:=\mathbf{H}_{T}^{\frac{1}{2}}(\partial \Omega) \times \mathbf{H}_{T}^{\frac{3}{2}}(\partial \Omega) \times H^{\frac{3}{2}}(\partial \Omega) \times H^{\frac{7}{2}}(\partial \Omega) .
$$

Lemma 4.1 The mapping

$$
\mathbf{H}^{4}(\Omega) \longrightarrow R^{0} \times S^{0}: \mathbf{u} \rightarrow(B \mathbf{u}, T \mathbf{u})^{\top}
$$

is surjective.

Proof. We first notice that using a permutation matrix from $\mathbb{C}^{16}$ into itself and for all $\mathbf{x} \in \partial \Omega$, the invertible relation

$$
Q_{\mathbf{x}}:\left\{\mathbf{y} \in \mathbb{C}^{3}: \mathbf{y} \cdot \mathbf{n}(\mathbf{x})=0\right\} \times \mathbb{C} \rightarrow \mathbb{C}^{3}:(\mathbf{y}, z)^{\top} \mapsto-\mathbf{y} \times \mathbf{n}(\mathbf{x})+z \mathbf{n}(\mathbf{x}),
$$

that yields $Q_{\mathbf{x}}(\mathbf{u} \times \mathbf{n}, \mathbf{u} \cdot \mathbf{n})^{\top}=\mathbf{u}$, there exists an invertible mapping $P$ from $R^{0} \times S^{0}$ to $\mathbf{R}:=$ $\mathbf{H}^{\frac{7}{2}}(\partial \Omega) \times \mathbf{H}^{\frac{5}{2}}(\partial \Omega) \times \mathbf{H}^{\frac{3}{2}}(\partial \Omega) \times \mathbf{H}^{\frac{1}{2}}(\partial \Omega)$ such that

$$
P(B \mathbf{u}, T \mathbf{u})^{\top}=(\gamma \mathbf{u}, \gamma \operatorname{curl} \mathbf{u} \times \mathbf{n}, \gamma \operatorname{div} \mathbf{u}, \gamma \Delta \mathbf{u}, \gamma \operatorname{curl} \Delta \mathbf{u} \times \mathbf{n}, \gamma \operatorname{div} \Delta \mathbf{u})^{\top} .
$$

Hence it suffices to show that the mapping

$$
M: \mathbf{H}^{4}(\Omega) \longrightarrow \mathbf{R}: \mathbf{u} \rightarrow P(B \mathbf{u}, T \mathbf{u})^{\top}
$$


is surjective. For that purpose, for all $\mathbf{x} \in \partial \Omega$, if we denote by $\left(\tau_{1}(\mathbf{x}), \tau_{2}(\mathbf{x})\right)$ two tangential vectors to $\partial \Omega$ at $\mathbf{x}$ such that $\left\{\tau_{1}(\mathbf{x}), \tau_{2}(\mathbf{x}), \mathbf{n}(\mathbf{x})\right\}$ forms an orthonormal basis of $\mathbb{R}^{3}$, we notice that

$$
\begin{aligned}
\operatorname{curl} \mathbf{u} \times \mathbf{n} & =\left(\mathbf{n} \times \partial_{n} \mathbf{u}\right) \times \mathbf{n}+\sum_{i=1}^{2}\left(\tau_{i} \times \frac{\partial \mathbf{u}}{\partial \tau_{i}}\right) \times \mathbf{n} \\
\operatorname{div} \mathbf{u} & =\mathbf{n} \cdot \partial_{n} \mathbf{u}+\sum_{i=1}^{2}\left(\tau_{i} \cdot \frac{\partial \mathbf{u}}{\partial \tau_{i}}\right)
\end{aligned}
$$

This means that the pair ( $\operatorname{curl} \mathbf{u} \times \mathbf{n}, \operatorname{div} \mathbf{u}$ ) is the sum of $\partial_{n} \mathbf{u}$ and tangential derivatives of $\mathbf{u}$, namely

$$
(\operatorname{curl} \mathbf{u} \times \mathbf{n}, \operatorname{div} \mathbf{u})=\partial_{n} \mathbf{u}+H_{1} \mathbf{u}
$$

where we can identify $\left(\left(\mathbf{n} \times \partial_{n} \mathbf{u}\right) \times \mathbf{n}, \mathbf{n} \cdot \partial_{n} \mathbf{u}\right)$ with $\partial_{n} \mathbf{u}$, because $\partial_{n} \mathbf{u}=\left(\mathbf{n} \times \partial_{n} \mathbf{u}\right) \times \mathbf{n}+\left(\mathbf{n} \cdot \partial_{n} \mathbf{u}\right) \mathbf{n}$, and we have set

$$
\left.H_{1} \mathbf{u}=\sum_{i=1}^{2}\left(\tau_{i} \times \frac{\partial \mathbf{u}}{\partial \tau_{i}}\right) \times \mathbf{n}, \tau_{i} \cdot \frac{\partial \mathbf{u}}{\partial \tau_{i}}\right)
$$

that is only made of tangential derivatives of $\mathbf{u}$. This observation implies that

$$
M \mathbf{u}=\left(\mathbf{u}, \partial_{n} \mathbf{u}+H_{1} \mathbf{u}, \Delta \mathbf{u}, \partial_{n} \Delta \mathbf{u}+H_{1} \Delta \mathbf{u}\right) .
$$

Since Leibniz's rule yields

$$
\partial_{n} \Delta \mathbf{u}=\Delta \partial_{n} \mathbf{u}-(\Delta \mathbf{n} \cdot \mathbf{n}) \partial_{n} \mathbf{u}-\sum_{i=1}^{2}\left(\Delta \mathbf{n} \cdot \tau_{i}\right) \frac{\partial \mathbf{u}}{\partial \tau_{i}},
$$

we get

$$
\partial_{n} \Delta \mathbf{u}=\partial_{n}^{3} \mathbf{u}+H_{2} \partial_{n} \mathbf{u}+H_{3} \mathbf{u}
$$

where

$$
\begin{aligned}
& H_{2} \mathbf{u}=\left(H_{4}-(\Delta \mathbf{n} \cdot \mathbf{n})\right) \mathbf{u} \\
& H_{3} \mathbf{u}=-\sum_{i=1}^{2}\left(\Delta \mathbf{n} \cdot \tau_{i}\right) \frac{\partial \mathbf{u}}{\partial \tau_{i}}, \\
& H_{4} \mathbf{u}=\sum_{i=1}^{2} \frac{\partial^{2}}{\partial \tau_{i}^{2}} \mathbf{u}
\end{aligned}
$$

are only made of tangential derivatives of $\mathbf{u}$.

In summary, we see that

$$
M \mathbf{u}=D \mathbf{u}+\left(\mathbf{0}, H_{1} \mathbf{u}, H_{4} \mathbf{u},\left(H_{3}+H H_{4}\right) \mathbf{u}+H_{2} \partial_{n} \mathbf{u}+H_{1} \partial_{n}^{2} \mathbf{u}\right)
$$

and consequently

$$
M \mathbf{u}=\left(\mathbf{h}_{0}, \mathbf{h}_{1}, \mathbf{h}_{2}, \mathbf{h}_{3}\right) \in \mathbf{R},
$$

if and only if

$$
D \mathbf{u}=\left(\mathbf{g}_{0}, \mathbf{g}_{1}, \mathbf{g}_{2}, \mathbf{g}_{3}\right) \in \mathbf{R},
$$


with the (invertible) relation

$$
\begin{aligned}
& \mathbf{g}_{0}=\mathbf{h}_{0}, \\
& \mathbf{g}_{1}=\mathbf{h}_{1}-H_{1} \mathbf{h}_{0}, \\
& \mathbf{g}_{2}=\mathbf{h}_{2}-H_{4} \mathbf{h}_{0}, \\
& \mathbf{g}_{3}=\mathbf{h}_{3}-\left(H_{3}+H H_{4}\right) \mathbf{h}_{0}-H_{2} \mathbf{h}_{1}-H \mathbf{h}_{2} .
\end{aligned}
$$

As a standard trace theorem (see for instance [16, Thm 1.5.1.2]) shows that the mapping $D$ is surjective from $\mathbf{H}^{4}(\Omega)$ onto $\mathbf{R}, M$ inherits the same property.

Clearly there exist two $8 \times 12$ matrices $Q$ and $R$ of tangential differential operators such that

$$
B \mathbf{u}=Q D \mathbf{u}, T \mathbf{u}=R D \mathbf{u}, \forall \mathbf{u} \in \mathbf{H}^{4}(\Omega) .
$$

Let $Q^{+}$(resp. $\left.R^{+}\right)$denote the adjoint operator of $Q$ (resp. $R$ ) in the sense that

$$
\int_{\partial \Omega} Q \mathbf{u} \cdot \mathbf{v}=\int_{\partial \Omega} \mathbf{u} \cdot Q^{+} \mathbf{v}, \int_{\partial \Omega} R \mathbf{u} \cdot \mathbf{v}=\int_{\partial \Omega} \mathbf{u} \cdot R^{+} \mathbf{v}, \forall \mathbf{u} \in C^{\infty}(\partial \Omega)^{12}, \mathbf{v} \in\left(C^{\infty}(\partial \Omega)\right)_{T}^{8},
$$

where

$$
\left(C^{\infty}(\partial \Omega)\right)_{T}^{8}=\left\{\left(\mathbf{v}_{1}, \mathbf{v}_{2}, \mathbf{v}_{3}, \mathbf{v}_{4}\right) \in C^{\infty}(\partial \Omega)^{8}: \mathbf{v}_{1} \cdot \mathbf{n}=\mathbf{v}_{2} \cdot \mathbf{n}=0\right\} .
$$

Then we introduce the boundary operator

$$
P=R^{+} B-Q^{+} T \text {. }
$$

We are ready to state the next Green formulas.

Lemma 4.2 For any $\mathbf{u} \in \mathbf{H}^{4}(\Omega), \mathbf{v} \in \mathbf{H}^{2}(\Omega)$, we have

$$
\text { (4.7) } \begin{aligned}
a(\mathbf{u}, \mathbf{v}) & =\int_{\Omega} \Delta^{2} \mathbf{u} \cdot \overline{\mathbf{v}} \\
& +\int_{\partial \Omega}(\operatorname{div} \Delta \mathbf{u} \overline{\mathbf{v}} \cdot \mathbf{n}-\Delta \mathbf{u} \cdot \mathbf{n} \operatorname{div} \overline{\mathbf{v}}-\operatorname{curl} \Delta \mathbf{u} \cdot(\overline{\mathbf{v}} \times \mathbf{n})-\Delta \mathbf{u} \cdot(\operatorname{curl} \overline{\mathbf{v}} \times \mathbf{n})) .
\end{aligned}
$$

Furthermore for any $\mathbf{u}, \mathbf{v} \in \mathbf{H}^{4}(\Omega)$, we have

$$
\begin{aligned}
\int_{\Omega} \Delta^{2} \mathbf{u} \cdot \overline{\mathbf{v}}+\int_{\partial \Omega} B \mathbf{u} \cdot T \overline{\mathbf{v}} & =\int_{\Omega} \mathbf{u} \cdot \Delta^{2} \overline{\mathbf{v}}+\int_{\partial \Omega} T \mathbf{u} \cdot B \overline{\mathbf{v}} \\
\int_{\Omega} \Delta^{2} \mathbf{u} \cdot \overline{\mathbf{v}} & =\int_{\Omega} \mathbf{u} \cdot \Delta^{2} \overline{\mathbf{v}}+\int_{\partial \Omega} D \mathbf{u} \cdot P \overline{\mathbf{v}} .
\end{aligned}
$$

Proof. The first identity is a simple application of standard Green's formulas (see for instance the identities (I.2.17) and (I.2.22) in [10]). The identity (4.8) follows from the first one by noticing that $a(\mathbf{u}, \mathbf{v})=\overline{a(\mathbf{v}, \mathbf{u})}$. The identity (4.9) is a re-writting of (4.8) by using the expressions (4.5) and (4.6) and reminding the definition of $Q^{+}$and $R^{+}$.

Now for any $\ell \in \mathbb{N}$, we introduce the operator

$$
A_{\ell}: \mathbf{H}^{4+\ell}(\Omega) \longrightarrow \mathbf{H}^{\ell}(\Omega) \times R^{\ell}: \mathbf{u} \rightarrow\left(\Delta^{2} \mathbf{u}, B \mathbf{u}\right),
$$

that is clearly a linear and continuous operator. By Theorem 4.2.4 of [21], $A_{\ell}$ is a Fredholm operator; its kernel consists of smooth functions and its cokernel is made of smooth functions 
$(\mathbf{v}, \underline{v})$ solution of the homogeneous adjoint problem (4.10) described below. Concerning the kernel of $A_{\ell}$, any $\mathbf{u} \in \operatorname{ker} A_{\ell}$ clearly belongs to $V$ and owing to (4.7), one obtains

$$
a(\mathbf{u}, \mathbf{u})=0 .
$$

By the coerciveness of $a$, we deduce that $\mathbf{u}=\mathbf{0}$ and consequently the kernel of $A_{\ell}$ is reduced to $\{\mathbf{0}\}$.

Let us go on with the characterization of the formal adjoint of $A_{\ell}$. Comparing the identity (4.9) with the identity (4.2.14) from [21], owing to the Definition 4.2 .3 of [21], we see that the formal adjoint of system $(3.9)$ consists in $(\mathbf{v}, \underline{v}) \in C^{\infty}(\Omega)^{3} \times\left(C^{\infty}(\partial \Omega)\right)_{T}^{8}$ solution of

$$
\begin{cases}\Delta^{2} \mathbf{v}=\mathbf{f} & \text { in } \Omega \\ P \mathbf{v}+Q^{+} \underline{v}=\mathbf{g} & \text { on } \partial \Omega\end{cases}
$$
$A_{0}$.

We now make the relationship between the kernel of this adjoint problem with the kernel of

Lemma $4.3(\mathbf{v}, \underline{v}) \in C^{\infty}(\Omega)^{3} \times\left(C^{\infty}(\partial \Omega)\right)_{T}^{8}$ is a solution of

$$
\begin{cases}\Delta^{2} \mathbf{v}=\mathbf{0} & \text { in } \Omega \\ P \mathbf{v}+Q^{+} \underline{v}=\mathbf{0} & \text { on } \partial \Omega\end{cases}
$$

if and only if

$$
\begin{cases}\Delta^{2} \mathbf{v}=\mathbf{0} & \text { in } \Omega \\ B \mathbf{v}=\mathbf{0} \text { and } \underline{v}=T \mathbf{v} & \text { on } \partial \Omega .\end{cases}
$$

Proof. From the definition of $P$, for all $\mathbf{u} \in \mathbf{H}^{4}(\Omega)$ and $(\mathbf{w}, \underline{w}) \in C^{\infty}(\Omega)^{3} \times\left(C^{\infty}(\partial \Omega)\right)_{T}^{8}$, we have

$$
\int_{\partial \Omega} D \mathbf{u} \cdot\left(P \overline{\mathbf{w}}+Q^{+} \underline{\underline{w}}\right)=\int_{\partial \Omega} T \mathbf{u} \cdot B \overline{\mathbf{w}}+B \mathbf{u} \cdot(\underline{\underline{w}}-T \overline{\mathbf{w}}) .
$$

Hence for a solution $(\mathbf{v}, \underline{v}) \in C^{\infty}(\Omega)^{3} \times\left(C^{\infty}(\partial \Omega)\right)_{T}^{8}$ of $(4.11)$, we get

$$
0=\int_{\partial \Omega} D \mathbf{u} \cdot\left(P \overline{\mathbf{v}}+Q^{+} \underline{\bar{v}}\right)=\int_{\partial \Omega}(B \mathbf{u}, T \mathbf{u}) \cdot(\underline{\bar{v}}-T \overline{\mathbf{v}}, B \overline{\mathbf{v}})=0, \forall \mathbf{u} \in \mathbf{H}^{4}(\Omega) .
$$

The conclusion follows, as Lemma 4.1 implies that the range of the mapping (4.2) is dense in $\left(\mathbf{L}_{T}^{2}(\partial \Omega) \times \mathbf{L}_{T}^{2}(\partial \Omega) \times L^{2}(\partial \Omega) \times L^{2}(\partial \Omega)\right)^{2}$.

Corollary 4.4 If $(\mathbf{v}, \underline{v}) \in C^{\infty}(\Omega)^{3} \times\left(C^{\infty}(\partial \Omega)\right)_{T}^{8}$ is a solution of $(4.11)$, then $(\mathbf{v}, \underline{v})=\mathbf{0}$.

In conclusion we have proved the next result.

Theorem 4.5 For all $\ell \in \mathbb{N}$, the operator $A_{\ell}$ is an isomorphism.

Corollary 4.6 Let $\mathbf{f} \in \mathbf{H}^{\ell}(\Omega)$, with $\ell \in \mathbb{N}$, then the weak solution $\mathbf{u} \in V$ of (3.3) belongs to $\mathbf{H}^{4+\ell}(\Omega)$.

Proof. By Theorem 4.5, there exists a unique $\mathbf{u} \in \mathbf{H}^{4+\ell}(\Omega)$ of (3.9). But due to (4.7), we have

$$
a(\mathbf{u}, \mathbf{v})=\int_{\Omega} \mathbf{f} \cdot \overline{\mathbf{v}}, \forall \mathbf{v} \in V .
$$

Since $\mathbf{u}$ belongs to $V$, this means that $\mathbf{u}$ is the unique weak solution of (3.3). 


\section{$5 \quad$ Edge singularities}

In this section, we assume that $\Omega$ is a polyhedral domain. Our goal is to describe the edge singularities of problem (3.9). Let us then fix an edge $e$ of $\Omega$, then near an interior point of $e$, up to a translation and a rotation, $\Omega$ behaves like $W_{e}=C_{e} \times \mathbb{R}$ where $C_{e}$ is a two-dimensional cone centred at $(0,0)$ of opening $\omega_{e} \in(0,2 \pi)$, with $\omega_{e} \neq \pi$. Below we will also use the polar coordinates $(r, \theta)$ in $C_{e}$ centred at $(0,0)$. Let us recall that the set $\Lambda_{D i r}\left(C_{e}\right)$ of singular exponents of the Laplace operator with Dirichlet boundary conditions in $C_{e}$ is defined by

$$
\Lambda_{\text {Dir }}\left(C_{e}\right)=\left\{\frac{k \pi}{\omega_{e}}: k \in \mathbb{Z} \backslash\{0\}\right\} .
$$

For convenience, when no confusion is possible, we will drop the index $e$. As usual, for $\lambda \in \mathbb{C}$, the edge singularities are obtained by looking for a non-polynomial solution $\mathbf{u}=r^{\lambda} \sum_{q=0}^{Q}(\ln r)^{q} \varphi_{q}(\theta)$ (hence independent of the $x_{3}$ variable) of (cfr. Theorem 3.1 and (3.9))

$$
\begin{cases}\Delta^{2} \mathbf{u}=\mathbf{F} & \text { in } C \times \mathbb{R} \\ \operatorname{div} \mathbf{u}=\Delta \operatorname{div} \mathbf{u}=0 & \text { on } \partial C \times \mathbb{R} \\ \mathbf{u} \times \mathbf{n}=(\operatorname{curl} \mathbf{u}) \times \mathbf{n}=0 & \text { on } \partial C \times \mathbb{R}\end{cases}
$$

$\mathbf{F}=\left(\mathbf{f}, f_{3}\right)$ being a polynomial in the $x_{1}, x_{2}$ variables. In that way, we see that the third component $u_{3}$ satisfies

$$
\begin{cases}\Delta^{2} u_{3}=f_{3} & \text { in } C, \\ u_{3}=\frac{\partial u_{3}}{\partial n}=0 & \text { on } \partial C,\end{cases}
$$

while the first two components $\mathbf{v}=\left(u_{1}, u_{2}\right)$ satisfies

$$
\begin{cases}\Delta^{2} \mathbf{v}=\mathbf{f} & \text { in } C \\ \operatorname{div} \mathbf{v}=\Delta \operatorname{div} \mathbf{v}=0 & \text { on } \partial C, \\ \mathbf{v} \cdot \mathbf{t}=\operatorname{curl} \mathbf{v}=0 & \text { on } \partial C\end{cases}
$$

that is the two-dimensional version of $(5.1)^{1}$.

The singularities of problem (5.2) are described in [16, Chap. 7] for instance (see also [28]), where it is shown that a function $u_{3}$ of the form $r^{\lambda} \varphi(\theta)$ is a solution of (5.2) with $f_{3}=0$ if and only if $\lambda$ is a root of

$$
\sin ^{2}((\lambda-1) \omega)=(\lambda-1)^{2} \sin ^{2} \omega
$$

It is shown in $[9, \S 5.1]$ (see also [16, Lemma 7.3.2.4]) that the strip $\Re \lambda \in\left[\frac{1}{2}, \frac{3}{2}\right]$ does not contain roots of (5.4) except $\lambda=1$ if $\omega \in(0,2 \pi)$, while the strip $\Re \lambda \in\left[1-\frac{\pi}{\omega}, 1+\frac{\pi}{\omega}\right]$ does not contain roots of (5.4) except $\lambda=1$ if $\omega \in(0, \pi)$. The case when $f_{3}$ is a non-zero polynomial corresponds to integer exponents and will be treated below.

As in [7], the singularities of system (5.3) are obtained by introducing the scalar variables $q=\operatorname{div} \mathbf{v}$ and $\psi=\operatorname{curl} \mathbf{v}$. In this way, we find the equivalent system

$$
\begin{gathered}
\Delta^{2} q=\operatorname{div} \mathbf{f} \text { in } C, \text { with } q=\Delta q=0 \text { on } \partial C, \\
\Delta \text { curl } \psi=-\nabla \Delta q \text { in } C, \text { with } \psi=0 \text { on } \partial C, \\
\text { curl } \mathbf{v}=\psi, \operatorname{div} \mathbf{v}=q \text { in } C, \text { with } \mathbf{v} \cdot \mathbf{t}=0 \text { on } \partial C .
\end{gathered}
$$

\footnotetext{
${ }^{1}$ in the whole section, except in the last Theorem 5.8 , when the operators $\nabla$, div, curl and $\Delta$ are applied to functions of the variables $x_{1}$ and $x_{2}$, they correspond to the two-dimensional operators
} 


\section{$5.1 \quad$ Non-integer exponents}

If $\lambda$ is not an integer, then $\mathbf{f}=\mathbf{0}$ and three types of singularities appear for system (5.5):

Type 1: $q=0, \psi=0$ and $\mathbf{v}$ general non-zero solution of (5.5c).

Type 2: $q=0, \psi$ general non-zero solution of $(5.5 \mathrm{~b})$ and $\mathbf{u}$ particular solution of $(5.5 \mathrm{c})$.

Type 3: $q$ general non-zero solution of $(5.5 \mathrm{a}), \psi$ particular solution of $(5.5 \mathrm{~b})$ and $\mathbf{u}$ particular solution of $(5.5 \mathrm{c})$.

The singularities of type 1 correspond exactly to the singularities of type 1 in $[7, \S 5$.b], where it is shown that $\lambda$ is such that $\lambda+1 \in \Lambda_{\text {Dir }}(C)$ and $\mathbf{v}=\nabla \Phi$ with $\Phi=r^{\lambda+1} \sin ((\lambda+1) \theta)$, as $\lambda \notin \mathbb{Z}$.

Let us now analyze the singularities of types 2 and 3 .

Lemma 5.1 If $\omega \neq \frac{3 \pi}{2}, \lambda \notin \mathbb{Z}$ is a singular exponent of type 2 of the system (5.3) if and only if $\lambda-1 \in \Lambda_{D i r}(C)$. If $\omega=\frac{3 \pi}{2}$, there is no non-integral singular exponents of type 2 for system (5.3).

Proof. For a singularity of type 2 , as $q=0$, by (5.5b), $\psi$ satisfies

$$
\Delta \operatorname{curl} \psi=0 \text { in } C \text {, with } \psi=0 \text { on } \partial C \text {. }
$$

Since $\Delta \operatorname{curl} \psi=\operatorname{curl} \Delta \psi$, we deduce that there exists a constant $c$ such that

$$
\Delta \psi=c \text { in } C .
$$

As $\Delta \psi$ behaves like $r^{\lambda-3}$ and we look for non-integer exponent $\lambda, c$ has to be zero. Therefore $\psi$ is solution of

$$
\Delta \psi=0 \text { in } C \text {, with } \psi=0 \text { on } \partial C .
$$

This means that a non-zero solution $\psi$ exists if and only if $\lambda-1 \in \Lambda_{D i r}(C)$ and

$$
\psi=r^{\lambda-1} \sin ((\lambda-1) \theta) .
$$

Now by (5.5c), $\mathbf{v}$ has to satisfy

$$
\text { curl } \mathbf{v}=r^{\lambda-1} \sin ((\lambda-1) \theta), \operatorname{div} \mathbf{v}=0 \text { in } C, \text { with } \mathbf{v} \cdot \mathbf{t}=0 \text { on } \partial C .
$$

But we readily check that the function $\mathbf{v}_{0}=\frac{1}{\lambda+1}\left(-x_{2} \psi, x_{1} \psi\right)$ satisfies

$$
\operatorname{curl} \mathbf{v}_{0}=\psi \text {. }
$$

Therefore by setting $\mathbf{v}_{1}:=\mathbf{v}-\mathbf{v}_{0},(5.7)$ is equivalent to

$$
\operatorname{curl} \mathbf{v}_{1}=0, \operatorname{div} \mathbf{v}_{1}=-\operatorname{div} \mathbf{v}_{0} \text { in } C, \text { with } \mathbf{v}_{1} \cdot \mathbf{t}=0 \text { on } \partial C .
$$

As $C$ is simply connected, there exists a scalar function $\Phi$ such that $\mathbf{v}_{1}=\nabla \Phi$ so that (5.7) reduces to

$$
\Delta \Phi=-\operatorname{div} \mathbf{v}_{0} \text { in } C, \text { with } \Phi=0 \text { on } \partial C .
$$

As $\operatorname{div} \mathbf{v}_{0}=r^{\lambda-1} \psi(\theta)$ for some smooth function $\psi$, we deduce that this problem has a solution $\Phi$ in the form $r^{\lambda+1} \varphi(\theta)$ if and only if $\lambda+1$ does not belong to $\Lambda_{D i r}(C)$ or $\lambda+1$ belongs to $\Lambda_{D i r}(C)$ but

$$
\int_{0}^{\omega} \psi(\theta) \sin ((\lambda+1) \theta) d \theta=0
$$


We now notice that $\lambda-1$ and $\lambda+1$ both belong to $\Lambda_{D i r}(C)$ if and only if $\omega=\frac{\pi}{2}$ or $\frac{3 \pi}{2}$. Hence if $\omega \notin\left\{\frac{\pi}{2}, \frac{3 \pi}{2}\right\}$, we find a solution $\Phi$ and hence $\mathbf{v}$. On the contrary, if $\omega=\frac{3 \pi}{2}$, then easy calculations show that (5.10) does not hold, and therefore $\lambda$ is not a singular exponent. Note that the case $\omega=\frac{\pi}{2}$ leads to integral exponents and is here excluded.

Lemma 5.2 If $\omega \neq \frac{3 \pi}{2}, \lambda \notin \mathbb{Z}$ is a singular exponent of type 3 of the system (5.3) if and only if

$$
\lambda-1 \in \Lambda_{\text {Dir }}(C) \text { or } \lambda-3 \in \Lambda_{\text {Dir }}(C) .
$$

If $\omega=\frac{3 \pi}{2}, \lambda \notin \mathbb{Z}$ is a singular exponent of type 3 of the system (5.3) if and only if $\lambda-1 \in \Lambda_{\text {Dir }}(C)$.

Proof. For a singularity of type $3, q=r^{\lambda-1} Q(\theta)$ is a general solution of (5.5a), i.e.,

$$
\Delta^{2} q=0 \text { in } C \text {, with } q=\Delta q=0 \text { on } \partial C .
$$

By the results from $[25, \S 3.2 .2]$, a non zero solution $q$ exists if and only if either $\lambda-1$ belongs to $\Lambda_{D i r}(C)$ and $Q(\theta)=\sin ((\lambda-1) \theta)$ or $\lambda-3$ belongs to $\Lambda_{D i r}(C)$ and $Q(\theta)=\sin ((\lambda-3) \theta)$.

Let us now treat the two cases separately.

a) If $\lambda-1 \in \Lambda_{D i r}(C)$, then $q$ is harmonic and therefore, we can take $\psi=0$. Hence $(5.5 \mathrm{c})$ becomes

$$
\text { curl } \mathbf{v}=0, \operatorname{div} \mathbf{v}=r^{\lambda-1} \sin ((\lambda-1) \theta) \text { in } C, \text { with } \mathbf{v} \cdot \mathbf{t}=0 \text { on } \partial C .
$$

As before this means that $\mathbf{v}=\nabla \Phi$ with

$$
\Delta \Phi=r^{\lambda-1} \sin ((\lambda-1) \theta) \text { in } C \text {, with } \Phi=0 \text { on } \partial C
$$

A solution $\Phi=r^{\lambda+1} \varphi(\theta)$ always exists. Indeed either $\lambda+1 \notin \Lambda_{D i r}(C)$ and then it is direct, or $\lambda+1 \in \Lambda_{D i r}(C)$ and its existence follows from the orthogonality property:

$$
\int_{0}^{\omega} \sin ((\lambda-1) \theta) \sin ((\lambda+1) \theta) d \theta=0 .
$$

b) If $\lambda-3 \in \Lambda_{D i r}(C)$, then $q$ is no more harmonic, but

$$
\Delta q=4(\lambda-1) S_{\text {Dir }},
$$

with $S_{\text {Dir }}=r^{\lambda-3} \sin ((\lambda-3) \theta)$, hence $(5.5 \mathrm{~b})$ becomes

$$
\text { curl } \Delta \psi=-4(\lambda-1) \nabla S_{\text {Dir }} \text { in } C \text {, with } \psi=0 \text { on } \partial C \text {. }
$$

But simple calculations show that

$$
\nabla S_{\text {Dir }}=-\operatorname{curl} S_{\mathrm{Neu}}
$$

with $S_{\mathrm{Neu}}=r^{\lambda-3} \cos ((\lambda-3) \theta)$. Consequently

$$
\operatorname{curl} \Delta \psi=4(\lambda-1) \operatorname{curl} S_{\mathrm{Neu}} \text { in } C \text {, }
$$

and since $\lambda$ is not an integer, $\psi$ is solution of

$$
\Delta \psi=4(\lambda-1) S_{\mathrm{Neu}} \text { in } C \text {, with } \psi=0 \text { on } \partial C .
$$


If $\lambda-1 \notin \Lambda_{D i r}(C)$, we find $\psi$ in the form $r^{\lambda-1} \Psi(\theta)$, otherwise (that holds if and only if $\omega \in\left\{\frac{\pi}{2}, \frac{3 \pi}{2}\right\}$ ) we did not find a solution in this form since

$$
\int_{0}^{\omega} \cos ((\lambda-3) \theta) \sin ((\lambda-1) \theta) d \theta \neq 0
$$

In the first case, by $(5.5 \mathrm{c}), \mathbf{v}$ has to satisfy

$$
\operatorname{curl} \mathbf{v}=\psi, \operatorname{div} \mathbf{v}=q \text { in } C, \text { with } \mathbf{v} \cdot \mathbf{t}=0 \text { on } \partial C .
$$

As $\mathbf{v}_{0}=\frac{1}{\lambda+1}\left(-x_{2} \psi, x_{1} \psi\right)$ satisfies curl $\mathbf{v}_{0}=\psi$, by setting $\mathbf{v}_{1}:=\mathbf{v}-\mathbf{v}_{0},(5.14)$ is equivalent to

$$
\text { curl } \mathbf{v}_{1}=0, \operatorname{div} \mathbf{v}_{1}=q-\operatorname{div} \mathbf{v}_{0} \text { in } C, \text { with } \mathbf{v}_{1} \cdot \mathbf{t}=0 \text { on } \partial C .
$$

As $C$ is simply connected, there exists a scalar function $\Phi$ such that $\mathbf{v}_{1}=\nabla \Phi$ so that (5.15) reduces to

$$
\Delta \Phi=q-\operatorname{div} \mathbf{v}_{0} \text { in } C \text {, with } \Phi=0 \text { on } \partial C .
$$

As before, we deduce that this problem has a solution in the form $r^{\lambda+1} \varphi(\theta)$ if either $\lambda+1$ does not belong to $\Lambda_{D i r}(C)$ or if $\lambda+1$ belongs to $\Lambda_{D i r}(C)$ with the constraint

$$
\int_{0}^{\omega} \partial_{\theta} \Psi(\theta) \sin ((\lambda+1) \theta) d \theta=0
$$

since an easy calculation shows that

$$
q-\operatorname{div} \mathbf{v}_{0}=r^{\lambda-1}\left(\sin ((\lambda-3) \theta)-\frac{\lambda-1}{\lambda+1} \partial_{\theta} \Psi(\theta)\right) .
$$

The second case holds if and only if both $\lambda-3$ and $\lambda+1$ belong to $\Lambda_{D i r}(C)$, which is possible only if $\omega=\frac{j \pi}{4}$, for $j=1, \cdots, 7$ and in that case, $\lambda=3+\frac{4 k}{j}$, with $k \in \mathbb{Z}^{\star}$. In such a situation, we have to check if (5.16) holds or not. To do so, as $\lambda-1$ is not in $\Lambda_{D i r}(C)$, we notice that

$$
\Psi(\theta)=\alpha\left[(\cos ((\lambda-3) \theta)-\cos ((\lambda-1) \theta))+\frac{\sin ((\lambda-1) \theta)}{\sin ((\lambda-1) \omega)}(\cos ((\lambda-3) \omega)-\cos ((\lambda-1) \omega))\right],
$$

with $\alpha=\frac{1}{4(\lambda-2)}$. This expression and simple calculations allow to show that

$$
\int_{0}^{\omega} \partial_{\theta} \Psi(\theta) \sin ((\lambda+1) \theta) d \theta=0
$$

if $\omega=\frac{j \pi}{4}$, for $j=3,5$ or 7 . Since the other cases are excluded, the proof is complete.

\subsection{Integer exponents}

If $\lambda$ is a non negative integer, again three types of singularities appear for system (5.5):

Type 1: $q, \psi$ polynomial and $\mathbf{v}$ general non-polynomial solution of $(5.5 \mathrm{c})$.

Type 2: $q$ polynomial, $\psi$ general non-polynomial solution of $(5.5 \mathrm{~b})$ and $\mathbf{u}$ particular solution of (5.5c).

Type 3: $q$ general non-polynomial solution of $(5.5 \mathrm{a}), \psi$ particular solution of $(5.5 \mathrm{~b})$ and $\mathbf{u}$ particular solution of $(5.5 \mathrm{c})$. 
The singularities of type 1 were treated in $[7, \S 5 c]$, where it is shown that $\lambda$ is such that $\lambda+1 \in \Lambda_{D i r}(C) \backslash\{2\}$ and $\mathbf{v}=\nabla \Phi$ with $\Phi=r^{\lambda+1}(\ln r \sin ((\lambda+1) \theta)+\theta \cos ((\lambda+1) \theta))+p_{\lambda}$, where $p_{\lambda}$ is a polynomial of degree $\lambda$.

Let us go on with the other singularities. First for any $n \in \mathbb{N}$, we define $Q^{n}$ as the space of homogeneous polynomial of degree $n$, and set $Q^{n}=\{0\}$ if $n$ is a negative integer.

Lemma $5.3 \lambda \in \mathbb{N} \backslash\{0,1,2\}$ is a singular exponent of type 2 of the system (5.3) if and only if $\lambda-1 \in \Lambda_{D i r}(C) . \lambda \in\{0,1,2\}$ is not a singular exponent of type 2 of the system (5.3).

Proof. For a singularity of type 2 , as $q \in Q^{\lambda-1}$, by (5.5b), $\psi$ satisfies

$$
\Delta \operatorname{curl} \psi \in Q^{\lambda-4} \text { with } \psi=0 \text { on } \partial C \text {. }
$$

Since $\Delta \operatorname{curl} \psi=\operatorname{curl} \Delta \psi$, we deduce that

$$
\Delta \psi \in Q^{\lambda-3} \text { with } \psi=0 \text { on } \partial C,
$$

if $\lambda \geq 3$ and

$$
\Delta \psi=0 \text { with } \psi=0 \text { on } \partial C,
$$

if $\lambda<3$.

In the first case, a non-polynomial solution $\psi$ exists if and only if $\lambda-1 \in \Lambda_{D i r}(C)$ (see [8, $\S 13 . \mathrm{C}])$. Now by $(5.5 \mathrm{c}), \mathbf{v}$ has to satisfy

$$
\operatorname{curl} \mathbf{v}=\psi, \operatorname{div} \mathbf{v}=q \text { in } C, \text { with } \mathbf{v} \cdot \mathbf{t}=0 \text { on } \partial C,
$$

and the arguments of the end of the proof of Lemma 5.1 allow to conclude the existence of such a v.

The case $\lambda=1$ yields $\psi=0$, while in the case $\lambda=0$ (resp. 2), a non-zero solution $\psi$ exists if and only if $-1 \in \Lambda_{\text {Dir }}(C)$ (resp. $1 \in \Lambda_{D i r}(C)$ ), which is excluded by the assumption $\omega_{e} \neq \pi$.

Lemma 5.4 $\lambda \in\{0,1\}$ is a singular exponent of type 3 of system (5.3) if and only if $\lambda-3 \in$ $\Lambda_{\text {Dir }}(C)$. 2, 3 and 4 are not a singular exponent of type 3 of system (5.3). $\lambda \in \mathbb{N}$ with $\lambda \geq 5$ is a singular exponent of type 3 of system (5.3) if and only if (5.11) holds.

Proof. For a singularity of type $3, q$ has to be a general non-polynomial solution of (5.5a), i.e.,

$$
\Delta^{2} q \in Q^{\lambda-5}, \text { with } q=\Delta q=0 \text { on } \partial C .
$$

For $\lambda \leq 4$, this reduces to (5.12) and by the proof of Lemma 5.2, a non-zero solution $q$ exists if and only if $\lambda-1 \in \Lambda_{D i r}(C)$ or $\lambda-3 \in \Lambda_{D i r}(C)$. The case $\lambda-1 \in \Lambda_{D i r}(C)$ is either excluded or gives rise to a polynomial solution $q$. The case $\lambda-3 \in \Lambda_{D i r}(C)$ gives a non polynomial solution for $\lambda=0$ or 1 .

If $\lambda$ is $\geq 5$, we consider the mapping

$$
\Delta^{2}:\left\{q \in Q^{\lambda-1}: q=\Delta q=0 \text { on } \partial C\right\} \rightarrow Q^{\lambda-5}: q \rightarrow \Delta^{2} q .
$$

As $\left\{q \in Q^{\lambda-1}: q=\Delta q=0\right.$ on $\left.\partial C\right\}$ and $Q^{\lambda-5}$ have the same dimension, this mapping is onto if and only if it is injective. Since the injectivity holds if and only if (5.11) does not hold, we find a non-polynomial solution $q$ if and only if (5.11) holds.

The existence of $\psi$ and $\mathbf{v}$ is obtained with the help of the arguments of the proof of Lemma 5.2 .

Let us finish this subsection by looking at the integer singular exponents of problem (5.2). 
Lemma 5.5 $\lambda \in \mathbb{N}$ is a singular exponent of problem (5.2) if and only if $\lambda$ is a root of (5.4).

Proof. For $\lambda \in \mathbb{N}$ with $\lambda \leq 3$, the right-hand side $f_{3}$ of (5.2) is a polynomial if and only if $f_{3}=0$. Hence $\lambda$ is a root of (5.4) and the question is whether $u_{3}$ is a polynomial or not.

For $\lambda=0$ or $2, u_{3}$ is in the form

$$
u_{3}(r, \theta)=r^{\lambda}\left(c_{1}+c_{2} \theta+c_{3} \sin (2 \theta)+c_{4} \cos (2 \theta)\right),
$$

with $c_{i} \in \mathbb{C}, i=1,2,3,4$. But $c_{2}$ is different from zero, otherwise the boundary conditions in (5.2) would imply that $u_{3}$ is zero. Consequently $u_{3}$ is not a polynomial.

For $\lambda=1, u_{3}$ is in the form

$$
u_{3}(r, \theta)=r\left(c_{1} \sin \theta+c_{2} \theta \sin \theta+c_{3} \cos \theta+c_{4} \theta \cos \theta\right),
$$

with $c_{i} \in \mathbb{C}, i=1,2,3,4$. For the same reason as before, we can check that $\left(c_{2}, c_{4}\right) \neq(0,0)$, whence $u_{3}$ is not a polynomial.

Finally 3 is not a root of (5.4), because $\omega$ is different from $\pi$ and $2 \pi$.

In the case $\lambda \in \mathbb{N}$ with $\lambda \geq 4$, one easily shows that the set

$$
Q_{\Delta^{2}, \text { Dir }}^{\lambda}:=\left\{v \in Q^{\lambda}: v=\partial_{n} v=0 \text { on } \partial C\right\}
$$

has the same dimension than $Q^{\lambda-4}$. Therefore the operator

$$
Q_{\Delta^{2}, \text { Dir }}^{\lambda} \rightarrow Q^{\lambda-4}: v \rightarrow \Delta^{2} u
$$

is bijective if and only if $\lambda$ is not a root of (5.4). On the contrary as the kernel of the above operator is not reduced to zero, a non-polynomial solution of (5.2) with $f_{3} \in Q^{\lambda-4}$ always exists.

\subsection{Conclusion}

Up to now we did not take into account on the regularity constraints on $\mathbf{u}$ coming from the augmented variational formulation. For that purpose, we introduce the sets

$$
\begin{aligned}
L_{l o c}^{2}\left(\bar{C}^{*}\right) & :=\left\{v \in L_{l o c}^{2}(C): v \in L^{2}(C \cap B(0, R)), \forall R>0\right\}, \\
H_{l o c}^{1}\left(\bar{C}^{*}\right) & :=\left\{v \in H_{l o c}^{1}(C): v \in H^{1}(C \cap B(0, R)), \forall R>0\right\},
\end{aligned}
$$

their vectorial form being defined in the same way. Hence the additional constraints on the solution $\mathbf{u}$ of $(5.1)$ are

$$
\mathbf{u} \in \mathbf{L}_{l o c}^{2}\left(\bar{C}^{*}\right), \text { curl } \mathbf{u} \in \mathbf{L}_{l o c}^{2}\left(\bar{C}^{*}\right), \operatorname{curl} \operatorname{curl} \mathbf{u} \in \mathbf{L}_{l o c}^{2}\left(\bar{C}^{*}\right) \text { and } \operatorname{div} \mathbf{u} \in H_{l o c}^{1}\left(\bar{C}^{*}\right) .
$$

In terms of $\mathbf{v}$ and $u_{3}$, this means that

$$
u_{3} \in L_{l o c}^{2}\left(\bar{C}^{*}\right), \Delta u_{3} \in L_{l o c}^{2}\left(\bar{C}^{*}\right),
$$

and

$$
\mathbf{v} \in \mathbf{L}_{l o c}^{2}\left(\bar{C}^{*}\right), \operatorname{curl} \mathbf{v} \in H_{l o c}^{1}\left(\bar{C}^{*}\right), \operatorname{div} \mathbf{v} \in H_{l o c}^{1}\left(\bar{C}^{*}\right)
$$


These constraints eliminate some of the singular exponents highlighted before. Indeed the first constraint in (5.18) or (5.19) yields $\Re \lambda>-1$. The consequences of the other constraints are summarised in the next Theorem. Before, let us set

$$
\begin{array}{r}
S_{b}:=\{\lambda \in \mathbb{C}: \lambda \text { is a root of }(5.4) \text { with } \Re \lambda>1\}, \\
S_{1}:=\left\{\lambda \in \mathbb{R} \backslash\{1\}: \lambda>-1 \text { and } \lambda+1 \in \Lambda_{\text {Dir }}(C)\right\}, \\
S_{2}:=\left\{\lambda \in \mathbb{R} \backslash\{2\}: \lambda>1 \text { and } \lambda-1 \in \Lambda_{\text {Dir }}(C)\right\}, \\
S_{3}(\omega):=\left\{\lambda \in \mathbb{R} \backslash\{2,3,4\}: \lambda>1 \text { and } \lambda-3 \in \Lambda_{\text {Dir }}(C)\right\}, \text { if } \omega \neq \frac{3 \pi}{2},
\end{array}
$$

while $S_{3}\left(\frac{3 \pi}{2}\right)=\emptyset$.

Theorem 5.6 The set of singular exponents of system (5.3) with the constraints (5.18) and (5.19) is

$$
S_{b} \cup S_{1} \cup S_{2} \cup S_{3}(\omega) .
$$

Proof. For $u_{3}$, we have to see the consequence of the second constraint in (5.18). But we notice that $\Delta u_{3}$ behaves like $r^{\lambda-2}$ hence it is in $L_{l o c}^{2}\left(\bar{C}^{*}\right)$ if either $\Re \lambda>1$ or $\left(\lambda=1\right.$ with $\left.\Delta u_{3}=0\right)$. In this second case easy calculations from (5.17) yield

$$
\Delta u_{3}=2 r^{-1}\left(c_{2} \cos \theta-c_{4} \sin \theta\right) .
$$

Since the functions $\cos \theta$ and $\sin \theta$ are linearly independent (in $L^{2}(0, \omega)$ ), we have

$$
\int_{0}^{\omega}\left|c_{2} \cos \theta-c_{4} \sin \theta\right|^{2} d \theta>0,
$$

because $\left(c_{2}, c_{4}\right) \neq(0,0)$. Consequently $r^{-1}\left(c_{2} \cos \theta-c_{4} \sin \theta\right)$ cannot be in $L_{l o c}^{2}\left(\bar{C}^{*}\right)$, hence this singularity has to be excluded.

For $\mathbf{v}$, the second and third constraints in (5.19) means that

$$
q \in H_{l o c}^{1}\left(\bar{C}^{*}\right) \text { and } \psi \in H_{l o c}^{1}\left(\bar{C}^{*}\right) .
$$

This yields no more constraint than $\lambda>-1$ for the singularities of type 1 , on the contrary for singularities of types 2 or 3 , this implies that $\lambda>1$, hence the conclusion.

Corollary 5.7 For any $s \geq 2$, there is no edge singular exponent associated with $e \in \mathcal{E}$ in the strip $(-1, s-1]$ if and only if $\omega_{e}<\frac{\pi}{s}$.

Proof. For the set $S_{b}$, this follows from $[9, \S 5.1]$ that shows that for $\omega_{e} \in(0, \pi)$, any root $\lambda$ of (5.4) such that $\Re \lambda>1$ satisfies $\Re \lambda>1+\frac{\pi}{\omega_{e}}$. Now any $\lambda \in S_{1}$ is given by

$$
\lambda=\frac{k \pi}{\omega_{e}}-1,
$$

with $k \in \mathbb{N}^{*}$. Hence we find the condition

$$
\frac{\pi}{\omega_{e}}>s .
$$

Similarly any $\lambda \in S_{2}$ is given by

$$
\lambda=\frac{k \pi}{\omega_{e}}+1,
$$


with $k \in \mathbb{N}^{*}$ and we find here the condition

$$
\frac{\pi}{\omega_{e}}>s-2
$$

Finally, if $\omega_{e} \neq \frac{3 \pi}{2}, \lambda \in S_{3}$ is given by

$$
\lambda=\frac{k \pi}{\omega_{e}}+3,
$$

with $k \in \mathbb{Z}^{*}$ such that $k>-\frac{2 \omega_{e}}{\pi}$. Since we already have found the constraint $\omega_{e}<\frac{\pi}{s}$, the quantity $-\frac{2 \omega_{e}}{\pi}$ is larger than $-\frac{2}{s}>-1$. Hence only positive integers $k$ have to be considered and for such $k$, we have

$$
\lambda=\frac{k \pi}{\omega_{e}}+3 \geq \frac{\pi}{\omega_{e}}+3>s+3 .
$$

From this result we will deduce that in the case of a polyhedral domain, the variational solution of problem (3.3) does not belong to $\mathbf{H}^{3}(\Omega)$ in general, namely we show the next result.

Theorem 5.8 Assume that $\Omega$ is a polyhedral domain. Then there exists $\mathbf{F} \in \mathbf{H}^{-1}(\Omega) \cap V^{\prime}$ such that the solution $\mathbf{u} \in V$ of

$$
a(\mathbf{u}, \mathbf{v})=\mathbf{F}(\mathbf{v}), \forall \mathbf{v} \in V
$$

does not belong to $\mathbf{H}^{3}(\Omega)$.

Proof. We first show that there always exists an edge $e$ such that $\omega_{e} \geq \frac{\pi}{3}$. Indeed for a fixed corner $c$ of $\Omega$, consider the section $G_{c}$ of the cone $G_{c}$ that coincides with $\Omega$ near $c$ (see section 6 below). This section has $N_{c}$ corners that corresponds to the edges $e_{i}, i=1, \cdots, N_{c}$, of $\Omega$ having $c$ as extremities. Then by the local Gauss-Bonnet theorem, we have

$$
\sum_{i=1}^{N_{c}} \omega_{e_{i}}=\left(N_{c}-2\right) \pi+\left|G_{c}\right|,
$$

where $\left|G_{c}\right|$ is the area of $G_{c}$. Hence $\omega_{\max }=\max _{i=1, \cdots, N_{c}} \omega_{e_{i}}$ satisfies

$$
N_{c} \omega_{\max } \geq\left(N_{c}-2\right) \pi,
$$

and since $N_{c} \geq 3$, we get $\omega_{\max } \geq \frac{\pi}{3}$, which proves the assertion.

If one edge $e$ has an opening $\omega_{e} \geq \frac{\pi}{2}$, by Lemma 3.4 (see also Remark 3.5), any element of $V$ is even not in $\mathbf{H}^{2}(\Omega)$. Hence we can now assume that $\Omega$ has one $e \in \mathcal{E}$ such that $\frac{\pi}{3} \leq \omega_{e}<\frac{\pi}{2}$ and set for shortness $\lambda=\frac{\pi}{\omega_{e}}-1$ that belongs to $(1,2]$. From our previous considerations, we know that the function $\mathbf{U}^{\lambda}=\left(\nabla_{2}\left(r^{\lambda+1} \sin ((\lambda+1) \theta)\right), 0\right)^{\top}$ is an edge singularity of our problem along $e^{2}$. To localize it, we fix a cut-off function $\eta_{0}$ depending only on $r$ and another cut-off function $\eta_{1}$ depending only on the $x_{3}$-variable (the edge one) such that $\eta_{1}=1$ near an interior point of $e$. Both are fixed with a sufficiently small support such that $\eta_{0} \eta_{1}$ is zero on all faces of $\Omega$ except the two ones having the edge $e$ in common.

\footnotetext{
${ }^{2}$ In this proof, the index 2 means the two-dimensional version of the differential operator, if no index is used then it is the standard three-dimensional operator
} 
In that way we consider $\eta_{0} \eta_{1} \mathbf{U}^{\lambda}$ that does not belong to $V$ because by $(2.3)$

$$
\operatorname{curl}\left(\eta_{0} \eta_{1} \mathbf{U}^{\lambda}\right)=\left(\eta_{1} \nabla_{2} \eta_{0}, \eta_{0} \partial_{3} \eta_{1}\right)^{\top} \times \mathbf{U}^{\lambda}
$$

which is not zero on the boundary of $\Omega$. Hence we need to correct it appropriately. Therefore we look for $\mathbf{r} \in \mathbf{H}^{3}(\Omega)$ such that

$$
\begin{cases}\mathbf{r}=\mathbf{0} & \text { on } \partial \Omega \\ \operatorname{div} \mathbf{r}=0 & \text { on } \partial \Omega \\ \operatorname{curl} \mathbf{r} \times \mathbf{n}=\operatorname{curl}\left(\eta_{0} \eta_{1} \mathbf{U}^{\lambda}\right) \times \mathbf{n} & \text { on } \partial \Omega\end{cases}
$$

But using the expressions (4.3) and (4.4) on each face, this system is equivalent to

$$
\begin{cases}\mathbf{r}=\mathbf{0} & \text { on } \partial \Omega \\ \mathbf{n} \cdot \partial_{n} \mathbf{r}=0 & \text { on } \partial \Omega \\ \left(\mathbf{n} \times \partial_{n} \mathbf{r}\right) \times \mathbf{n}=\operatorname{curl}\left(\eta_{0} \eta_{1} \mathbf{U}^{\lambda}\right) \times \mathbf{n} & \text { on } \partial \Omega\end{cases}
$$

This means that it suffices to require that

$$
\begin{cases}\mathbf{r}=\mathbf{0} & \text { on } \partial \Omega, \\ \partial_{n} \mathbf{r}=\operatorname{curl}\left(\eta_{0} \eta_{1} \mathbf{U}^{\lambda}\right) \times \mathbf{n} & \text { on } \partial \Omega .\end{cases}
$$

But the identity (5.21) implies that the restriction of curl $\left(\eta_{0} \eta_{1} \mathbf{U}^{\lambda}\right) \times \mathbf{n}$ on each face $F$ of $\Omega$ belongs to $\mathbf{H}^{\frac{3}{2}}(F) \cap \mathbf{H}_{0}^{1}(F)$ and its tangential derivatives are in $\tilde{H}^{\frac{1}{2}}(F)^{3}$. Consequently by the trace theorem from [15], there exists $\mathbf{r} \in \mathbf{H}^{3}(\Omega)$ satisfying the boundary conditions (5.22).

Therefore the function

$$
\mathbf{u}^{\lambda}=\eta_{0} \eta_{1} \mathbf{U}^{\lambda}-\mathbf{r}
$$

belongs to $V$ but not in $\mathbf{H}^{3}(\Omega)$ because $\eta_{0} \eta_{1} \mathbf{U}^{\lambda}$ belongs to $\mathbf{H}^{2}(\Omega)$ but not to $\mathbf{H}^{3}(\Omega)$ since $\lambda \in(1,2]$. It then remains to show that it is the solution of problem (5.20) with an appropriated right-hand side. But Leibniz's rule and the fact that $\mathbf{U}^{\lambda}$ is harmonic imply that

$$
\Delta\left(\eta_{0} \eta_{1} \mathbf{U}^{\lambda}\right)=2 \eta_{1} \partial_{r} \eta_{0} \partial_{r} \mathbf{U}^{\lambda}+\eta_{1} \Delta_{2} \eta_{0} \mathbf{U}^{\lambda}+\eta_{0} \partial_{3}^{2} \eta_{1} \mathbf{U}^{\lambda}
$$

The assumption $\lambda>1$ guarantees that $\eta_{0} \partial_{3}^{2} \eta_{1} \mathbf{U}^{\lambda}$ belongs to $\mathbf{H}^{2}(\Omega)$ and since $\mathbf{U}^{\lambda}$ is smooth far away from the edge, we deduce that $\Delta\left(\eta_{0} \eta_{1} \mathbf{U}^{\lambda}\right)$ belongs to $\mathbf{H}^{2}(\Omega)$. With the regularity of $\mathbf{r}, \Delta \mathbf{u}^{\lambda}$ then belongs to $\mathbf{H}^{1}(\Omega)$. Now for $\mathbf{v} \in V$, curl $\mathbf{v}$ belongs to $X_{N}(\Omega)$ and div $\mathbf{v}$ is in $H_{0}^{1}(\Omega)$, and Green's formulas (2.2) and (2.1) lead to

$$
\begin{aligned}
\int_{\Omega} \Delta \mathbf{u}^{\lambda} \cdot \operatorname{curl} \operatorname{curl} \mathbf{v} & =\int_{\Omega} \operatorname{curl} \Delta \mathbf{u}^{\lambda} \cdot \operatorname{curl} \mathbf{v}, \\
\int_{\Omega} \Delta \mathbf{u}^{\lambda} \cdot \nabla \operatorname{div} \mathbf{v} & =-\int_{\Omega} \operatorname{div} \Delta \mathbf{u}^{\lambda} \operatorname{div} \mathbf{v} .
\end{aligned}
$$

The difference of these two identities directly furnishes (5.20) with

$$
\mathbf{F}(\mathbf{v})=-\int_{\Omega}\left(\operatorname{curl} \Delta \mathbf{u}^{\lambda} \cdot \operatorname{curl} \mathbf{v}+\operatorname{div} \Delta \mathbf{u}^{\lambda} \operatorname{div} \mathbf{v}\right),
$$

that indeed is in $\mathbf{H}^{-1}(\Omega) \cap V^{\prime}$.

Remark 5.9 For $\mathbf{F} \in \mathbf{H}^{-1}(\Omega) \cap V^{\prime}$ the maximal regularity that the variational solution $\mathbf{u}$ of (5.20) can have is $\mathbf{H}^{3}(\Omega)$ because $\Delta^{2} \mathbf{u}=\mathbf{F}$ in $\mathcal{D}^{\prime}(\Omega)^{3}$. Theorem 5.8 asserts that such a maximal regularity does not hold in general. Furthermore if $\Omega$ has an edge $e$ with $\omega_{e} \in\left[\frac{\pi}{4}, \frac{\pi}{3}\right)$, then similar arguments show that there exists $\mathbf{f} \in \mathbf{L}^{2}(\Omega)$ such that the solution $\mathbf{u} \in V$ of problem (3.3) does not belong to $\mathbf{H}^{4}(\Omega)$. 


\section{Corner singularities}

In the case 2 or 3 , let $c$ be a corner of $\Omega, \Gamma_{c}$ be the three-dimensional cone that coincides with $\Omega$ in a neighbourhood of $c$ and let $G_{c}$ be its section with the unit sphere. For shortness, if no confusion is possible, we will drop the index $c$. As usual denote by $(r, \vartheta)$ the spherical coordinates centred at $c$. Then we look for corner singularities $\mathbf{u}$ in the form $\mathbf{u}=r^{\lambda} \mathbf{U}(\vartheta)$, with $\lambda \in \mathbb{C}$ such that $\Re \lambda>-1$ and $\mathbf{U} \in \mathbf{L}^{2}(G)$ that is solution of

$$
\begin{cases}\Delta^{2} \mathbf{u}=\mathbf{0} & \text { in } \Gamma, \\ \operatorname{div} \mathbf{u}=\Delta \operatorname{div} \mathbf{u}=0 & \text { on } \partial \Gamma, \\ \mathbf{u} \times \mathbf{n}=(\operatorname{curl} \mathbf{u}) \times \mathbf{n}=0 & \text { on } \partial \Gamma\end{cases}
$$

As in [7], we introduce the auxilliary variables $q=\operatorname{div} \mathbf{u}$ and $\psi=$ curl $\mathbf{u}$ and can re-write the above system in the equivalent form

$$
\begin{gathered}
\Delta^{2} q=0 \text { in } \Gamma, \text { with } q=\Delta q=0 \text { on } \partial \Gamma, \\
\Delta \operatorname{curl} \psi=-\nabla \Delta q, \operatorname{div} \psi=0 \text { in } \Gamma, \text { with } \psi=0 \text { on } \partial \Gamma, \\
\text { curl } \mathbf{u}=\psi, \operatorname{div} \mathbf{u}=q \text { in } \Gamma, \text { with } \mathbf{u} \times \mathbf{n}=0 \text { on } \partial \Gamma .
\end{gathered}
$$

Then as in section 5 , three types of singularities appear:

Type 1: $q=0, \psi=0$ and $\mathbf{u}$ general non-zero solution of (6.2c). This case corresponds to singularities of type $1 \mathrm{in}[7]$ and are described in Lemma 6.4 of [7].

Type 2: $q=0, \psi$ general non-zero solution of (6.2b) and $\mathbf{u}$ particular solution of (6.2c).

Type 3: $q$ general non-zero solution of $(6.2 \mathrm{a}), \psi$ particular solution of $(6.2 \mathrm{~b})$ and $\mathbf{u}$ particular solution of $(6.2 \mathrm{c})$.

Remark 6.1 The general case where the right-hand side in the first identity of (6.1) is replaced by is a polynomial $\mathbf{F}$ of degree $\lambda-4$ is not treated here because for $\lambda \leq 4 \operatorname{div} \mathbf{F}=0$ (that corresponds to (6.2)) and the knowledge of the corner singular exponents in the strip $\Re \lambda \in\left(-\frac{3}{2}, 5\right)$ allows to analyze the regularity $\mathbf{H}^{s+2}(\Omega)$ of our solution up to $s+2<6.5$, that is more than the expected maximal regularity with a datum in $\mathbf{L}^{2}(\Omega)$. Furthermore the knowledge of the corner singular exponents of (6.1) allows to state regularity results in weighted Sobolev spaces (see section 7).

The singularities of types 2 and 3 are fully different from those from [7] and are described below. For that purpose, we recall the corner singularities of the Laplace operator with Dirichlet (resp. Neumann) boundary conditions in $\Gamma$, see $[16,8,7]$ for instance. We first denote by $L_{G}^{\text {Dir }}$ (resp. $L_{G}^{\mathrm{Neu}}$ ) the positive (resp. non-negative) Laplace-Beltrami operator with Dirichlet (resp. Neumann) boundary conditions on $G$. Recall that $L_{G}^{\mathrm{Dir}}$ and $L_{G}^{\mathrm{Neu}}$ are self-adjoint operators with a compact resolvent in $L^{2}(G)$, hence we denote by $\sigma\left(L_{G}^{\text {Dir }}\right)$ and $\sigma\left(L_{G}^{\text {Neu }}\right)$ their respective spectrum. Then we make the following definition

Definition 6.2 The set $\Lambda_{\text {Dir }}(\Gamma)$ of corner singular exponents of the Laplace operator with Dirichlet boundary conditions in $\Gamma$ is defined as the set of $\lambda \in \mathbb{C}$ such that there exists a non-trivial solution $\varphi \in H_{0}^{1}(G)$ of

$$
\Delta\left(r^{\lambda} \varphi(\vartheta)\right)=0 .
$$

We denote by $Z_{\text {Dir }}^{\lambda}$ the (finite) set of such solutions and fix a basis $\left\{u_{\operatorname{Dir}}^{\lambda, p}\right\}_{p=1}^{N_{\text {Dir }}(\lambda)}$, with $N_{\operatorname{Dir}}(\lambda) \in$ $\mathbb{N} \backslash\{0\}$. Similarly, the set $\Lambda_{\mathrm{Neu}}(\Gamma)$ of corner singular exponents of the Laplace operator with 
Neumann boundary conditions in $\Gamma$ is defined as the set of $\lambda \in \mathbb{C}$ different from -1 , such that there exists a solution $\varphi \in H^{1}(G)$ of (6.3) and the Neumann boundary condition:

$$
\partial_{n}\left(r^{\lambda} \varphi\right)=0 \text { on } \partial \Gamma
$$

We denote by $Z_{\mathrm{Neu}}^{\lambda}$ the set of such solutions and fix a basis $\left\{u_{\mathrm{Neu}}^{\lambda, p}\right\}_{p=1}^{N_{\mathrm{Neu}}(\lambda)}$, with $N_{\mathrm{Neu}}(\lambda) \in \mathbb{N} \backslash\{0\}$.

Due to the relation

$$
r^{2} \Delta=\left(r \partial_{r}\right)^{2}+\left(r \partial_{r}\right)+\Delta_{G},
$$

for any $\lambda \in \mathbb{C}$ and $\varphi \in H^{1}(G)$, we have

$$
\Delta\left(r^{\lambda} \varphi\right)=r^{\lambda-2} \mathcal{L}(\lambda) \varphi
$$

where

$$
\mathcal{L}(\lambda) \varphi=\Delta_{G} \varphi+\lambda(\lambda+1) \varphi,
$$

with $\Delta_{G}$ the Laplace-Beltrami operator on $G$. Consequently, the sets $\Lambda_{\operatorname{Dir}}(\Gamma)$ and $\Lambda_{\mathrm{Neu}}(\Gamma)$ are related to the spectrum of $L_{G}^{\mathrm{Dir}}$ and $L_{G}^{\mathrm{Neu}}$ as follows (see [7, Lemma 2.4]):

$$
\begin{aligned}
\Lambda_{\text {Dir }}(\Gamma) & =\left\{-\frac{1}{2} \pm \sqrt{\mu+\frac{1}{4}}: \mu \in \sigma\left(L_{G}^{\mathrm{Dir}}\right)\right\}, \\
\Lambda_{\mathrm{Neu}}(\Gamma) & =\left\{-\frac{1}{2} \pm \sqrt{\mu+\frac{1}{4}}: \mu \in \sigma\left(L_{G}^{\mathrm{Neu}}\right) \backslash\{0\}\right\} .
\end{aligned}
$$

For $\lambda \in \Lambda_{\text {Dir }}(\Gamma)$, the elements of $Z_{\text {Dir }}^{\lambda}$ are related to the set $V_{\text {Dir }}(\lambda)$ of eigenvectors of $L_{G}^{\text {Dir }}$ associated with $\mu=\lambda(\lambda+1)$ via the relation

$$
Z_{\text {Dir }}^{\lambda}=\left\{r^{\lambda} \varphi: \varphi \in V_{\text {Dir }}(\lambda)\right\} .
$$

The same holds for $\lambda \in \Lambda_{\mathrm{Neu}}(\Gamma)$, namely

$$
Z_{\mathrm{Neu}}^{\lambda}=\left\{r^{\lambda} \varphi: \varphi \in V_{\mathrm{Neu}}(\lambda)\right\}
$$

where $V_{\mathrm{Neu}}(\lambda)$ is the set of eigenvectors of $L_{G}^{\mathrm{Neu}}$ associated with $\mu=\lambda(\lambda+1)$.

Remark that for $\lambda \notin \Lambda_{\text {Dir }}(\Gamma)$, the operator $\mathcal{L}(\lambda)$ is an isomorphism from $H_{0}^{1}(G)$ into $H^{-1}(G)$, we then denote its inverse by $\mathcal{L}_{\text {Dir }}(\lambda)^{-1}$.

Now let us recall that $\nabla_{T}$ is the tangential component of the gradient on the unit sphere, while $\operatorname{div}_{T}$ is the adjoint of $-\nabla_{T}$, namely for a distribution $\mathbf{u}$, we define

$$
\left\langle\operatorname{div}_{T} \mathbf{u}, \varphi\right\rangle=-\int_{G} \mathbf{u} \cdot \nabla_{T} \varphi d \sigma, \forall \varphi \in \mathcal{D}(G) .
$$

Further for a vector field $\psi$ defined on $G$, we denote by $\psi_{r}=\psi \cdot \vartheta$ its radial component, while $\psi_{T}=\psi-\psi_{r} \vartheta$ is its angular component.

For any $\varphi \in H_{0}^{1}(G)$ and any $\lambda \in \mathbb{C}$, we recall that

$$
\nabla\left(r^{\lambda} \varphi\right)=r^{\lambda-1} g(\lambda) \varphi
$$

where for shortness, we have set

$$
g(\lambda) \varphi=\nabla_{T} \varphi+\lambda \varphi \vartheta .
$$


Similarly for $\mathbf{v} \in \mathbf{H}_{0}^{1}(G)$ and any $\lambda \in \mathbb{C}$, we notice that

$$
\begin{aligned}
& \operatorname{div}\left(r^{\lambda} \mathbf{v}\right)=r^{\lambda-1} d(\lambda) \mathbf{v} \\
& \operatorname{curl}\left(r^{\lambda} \mathbf{v}\right)=r^{\lambda-1} c(\lambda) \mathbf{v},
\end{aligned}
$$

where we have set

$$
d(\lambda) \mathbf{v}=\operatorname{div}_{T} \mathbf{v}_{T}+(\lambda+2) \mathbf{v} \cdot \vartheta, c(\lambda) \mathbf{v}=\nabla_{T} \times \mathbf{v}+\lambda \vartheta \times \mathbf{v} .
$$

Now for $\lambda \notin \Lambda_{\text {Dir }}(\Gamma)$, we introduce the operator $\mathcal{A}(\lambda)$ defined by

$$
\mathcal{A}(\lambda) \varphi=d(\lambda)\left(\mathcal{L}_{\text {Dir }}(\lambda)^{-1}(g(\lambda-1) \varphi)\right), \forall \varphi \in L^{2}(G) .
$$

For each $\lambda \notin \Lambda_{\text {Dir }}(\Gamma), \mathcal{A}(\lambda)$ is clearly a bounded linear operator from $L^{2}(G)$ into itself, further if $U=\mathbb{C} \backslash \Lambda_{\text {Dir }}(\Gamma)$, the mapping

$$
U \rightarrow \mathcal{L}\left(L^{2}(G)\right): \lambda \rightarrow \mathcal{A}(\lambda)
$$

is holomorphic. Let us now show that they form a holomorphic family of Fredholm operator of index zero.

Lemma 6.3 For all $\lambda, \lambda^{\prime} \in U, \mathcal{A}(\lambda)-\mathcal{A}\left(\lambda^{\prime}\right)$ is compact.

Proof. We split up

$$
\mathcal{A}(\lambda)=\operatorname{div}_{T}\left(\mathcal{L}_{\text {Dir }}(\lambda)^{-1} \nabla_{T}\right)_{T}+\mathcal{R}(\lambda) .
$$

The remainder $\mathcal{R}(\lambda)$ is a compact operator because for $\varphi \in L^{2}(G)$, we have

$$
\mathcal{R}(\lambda) \varphi=(\lambda-1) \operatorname{div}_{T}\left(\mathcal{L}_{\operatorname{Dir}}(\lambda)^{-1}(\varphi \vartheta)\right)_{T}+(\lambda+2)\left(\mathcal{L}_{\operatorname{Dir}}(\lambda)^{-1}\left(\nabla_{T} \varphi+(\lambda-1) \varphi \vartheta\right)\right)_{r} .
$$

Since $\varphi \vartheta$ belongs to $\mathbf{L}^{2}(G)$, there exists $\varepsilon>0$ small enough such that $\mathcal{L}_{\text {Dir }}(\lambda)^{-1}(\varphi \vartheta) \in \mathbf{H}^{\frac{3}{2}+\varepsilon}(G)$ with

$$
\left\|\mathcal{L}_{\operatorname{Dir}}(\lambda)^{-1}(\varphi \vartheta)\right\|_{\frac{3}{2}+\varepsilon, G} \lesssim\|\varphi \vartheta\|_{G} .
$$

Consequently $\operatorname{div}_{T}\left(\mathcal{L}_{\text {Dir }}(\lambda)^{-1}(\varphi \vartheta)\right)_{T}$ belongs to $H^{\frac{1}{2}+\varepsilon}(G)$, with

$$
\left\|\operatorname{div}_{T}\left(\mathcal{L}_{\operatorname{Dir}}(\lambda)^{-1}(\varphi \vartheta)\right)_{T}\right\|_{\frac{1}{2}+\varepsilon, G} \lesssim\|\varphi\|_{G} .
$$

Similarly as $\nabla_{T} \varphi+(\lambda-1) \varphi \vartheta$ belongs to $\mathbf{H}^{-1}(G)$, we deduce that $\mathcal{L}_{\operatorname{Dir}}(\lambda)^{-1}\left(\nabla_{T} \varphi+(\lambda-1) \varphi \vartheta\right)$ belongs to $\mathbf{H}_{0}^{1}(G)$ with

$$
\left\|\mathcal{L}_{\operatorname{Dir}}(\lambda)^{-1}\left(\nabla_{T} \varphi+(\lambda-1) \varphi \vartheta\right)\right\|_{1, G} \lesssim\|\varphi\|_{G} .
$$

This implies that $\mathcal{R}(\lambda) \varphi$ belongs to $H^{\frac{1}{2}+\varepsilon}(G)$, for some $\varepsilon \in(0,1 / 2)$, with

$$
\|\mathcal{R}(\lambda) \varphi\|_{\frac{1}{2}+\varepsilon, G} \lesssim\|\varphi\|_{G}
$$

The compact property of $\mathcal{R}(\lambda)$ directly follows.

Now as

$$
\mathcal{L}_{\text {Dir }}(\lambda)^{-1}-\mathcal{L}_{\text {Dir }}\left(\lambda^{\prime}\right)^{-1}=\left(\lambda(\lambda+1)-\lambda^{\prime}\left(\lambda^{\prime}+1\right)\right) \mathcal{L}_{\text {Dir }}(\lambda)^{-1} \mathcal{L}_{\text {Dir }}\left(\lambda^{\prime}\right)^{-1},
$$


we deduce that

$$
\mathcal{A}(\lambda)-\mathcal{A}\left(\lambda^{\prime}\right)=\left(\lambda(\lambda+1)-\lambda^{\prime}\left(\lambda^{\prime}+1\right)\right) \operatorname{div}_{T}\left(\mathcal{L}_{\mathrm{Dir}}(\lambda)^{-1} \mathcal{L}_{\mathrm{Dir}}\left(\lambda^{\prime}\right)^{-1} \nabla_{T}\right)_{T}+\mathcal{R}(\lambda)-\mathcal{R}\left(\lambda^{\prime}\right) .
$$

It then remains to show that $\operatorname{div}_{T}\left(\mathcal{L}_{\text {Dir }}(\lambda)^{-1} \mathcal{L}_{\text {Dir }}\left(\lambda^{\prime}\right)^{-1} \nabla_{T}\right)_{T}$ is compact.

But for any $\varphi \in L^{2}(G), \mathcal{L}_{\text {Dir }}\left(\lambda^{\prime}\right)^{-1} \nabla_{T} \varphi$ is in $\mathbf{H}_{0}^{1}(G)$ with

$$
\left\|\mathcal{L}_{\text {Dir }}\left(\lambda^{\prime}\right)^{-1} \nabla_{T} \varphi\right\|_{1, G} \lesssim\|\varphi\|_{G} .
$$

Therefore there exists $\varepsilon>0$ small enough such that $\mathcal{L}_{\text {Dir }}(\lambda)^{-1} \mathcal{L}_{\text {Dir }}\left(\lambda^{\prime}\right)^{-1} \nabla_{T} \varphi \in \mathbf{H}^{\frac{3}{2}+\varepsilon}(G)$ with

$$
\left\|\mathcal{L}_{\text {Dir }}(\lambda)^{-1} \mathcal{L}_{\text {Dir }}\left(\lambda^{\prime}\right)^{-1} \nabla_{T} \varphi\right\|_{\frac{3}{2}+\varepsilon, G} \lesssim\left\|\mathcal{L}_{\text {Dir }}\left(\lambda^{\prime}\right)^{-1} \nabla_{T} \varphi\right\|_{G} \lesssim\|\varphi\|_{G}
$$

This implies that

$$
\left\|\operatorname{div}_{T}\left(\mathcal{L}_{\operatorname{Dir}}(\lambda)^{-1} \mathcal{L}_{\operatorname{Dir}}\left(\lambda^{\prime}\right)^{-1} \nabla_{T} \varphi\right)_{T}\right\|_{\frac{1}{2}+\varepsilon, G} \lesssim\|\varphi\|_{G}
$$

and shows the compactness of the operator $\operatorname{div}_{T}\left(\mathcal{L}_{\operatorname{Dir}}(\lambda)^{-1} \mathcal{L}_{\operatorname{Dir}}\left(\lambda^{\prime}\right)^{-1} \nabla_{T}\right)_{T}$.

Lemma 6.4 If $\lambda \in U$ is real, the adjoint $\mathcal{A}(\lambda)^{*}$ of $\mathcal{A}(\lambda)$ is given by

$$
\mathcal{A}(\lambda)^{*}=\mathcal{A}(-(\lambda+1)) .
$$

Proof. We note that for any $\lambda \in U \cap \mathbb{R}$ and $\varphi, \chi \in L^{2}(G)$, we have

$$
\begin{aligned}
(\mathcal{A}(\lambda) \varphi, \chi)_{G} & =\left\langle\mathcal{L}_{\operatorname{Dir}}(\lambda)^{-1}\left(\nabla_{T} \varphi+(\lambda-1) \varphi \vartheta\right),-\nabla_{T} \chi+(\lambda+2) \chi \vartheta\right\rangle \\
& =-\left\langle\left(\nabla_{T} \varphi+(\lambda-1) \varphi \vartheta\right), \mathcal{L}_{\operatorname{Dir}}(\lambda)^{-1}\left(\nabla_{T} \chi-(\lambda+2) \chi \vartheta\right\rangle\right. \\
& =\left(\varphi, d(-(1+\lambda)) \mathcal{L}_{\operatorname{Dir}}(\lambda)^{-1}\left(\nabla_{T} \chi-(\lambda+2) \chi \vartheta\right\rangle\right)_{G} .
\end{aligned}
$$

Since $\mathcal{L}_{\text {Dir }}(\lambda)=\mathcal{L}_{\text {Dir }}(-(1+\lambda))$, the conclusion follows.

Lemma 6.5 $\mathcal{A}\left(-\frac{1}{2}\right)$ is an isomorphism.

Proof. Choosing $\lambda=-\frac{1}{2}$, we have $\lambda=-(\lambda+1)$, and owing to Lemma $6.4, \mathcal{A}\left(-\frac{1}{2}\right)$ is a selfadjoint and bounded operator. Hence it will be an isomorphism if we can show that (see for instance $[5$, Thm 2.20])

$$
\left(\mathcal{A}\left(-\frac{1}{2}\right) \varphi, \varphi\right)_{G} \gtrsim\|\varphi\|_{G}^{2}, \forall \varphi \in L^{2}(G) .
$$

But by the identity

$$
\left(\mathcal{A}\left(-\frac{1}{2}\right) \varphi, \chi\right)_{G}=-\left\langle\mathcal{L}_{\operatorname{Dir}}\left(-\frac{1}{2}\right)^{-1}\left(\nabla_{T} \varphi-\frac{3}{2} \varphi \vartheta\right), \nabla_{T} \chi-\frac{3}{2} \chi \vartheta\right\rangle
$$

and the fact that $-\mathcal{L}_{\operatorname{Dir}}\left(-\frac{1}{2}\right)$ is a positive selfadjoint operator, we deduce that

$$
\left(\mathcal{A}\left(-\frac{1}{2}\right) \varphi, \chi\right)_{G}=\left(\left(-\mathcal{L}_{\text {Dir }}\left(-\frac{1}{2}\right)\right)^{-\frac{1}{2}}\left(\nabla_{T} \varphi-\frac{3}{2} \varphi \vartheta\right),\left(-\mathcal{L}_{\text {Dir }}\left(-\frac{1}{2}\right)\right)^{-\frac{1}{2}}\left(\nabla_{T} \chi-\frac{3}{2} \chi \vartheta\right)\right)_{G} .
$$

As $\left(-\mathcal{L}_{\text {Dir }}\left(-\frac{1}{2}\right)\right)^{-\frac{1}{2}}$ is an isomorphism from $H^{-1}(G)$ to $L^{2}(G)$, we then get

$$
\left(\mathcal{A}\left(-\frac{1}{2}\right) \varphi, \varphi\right)_{G} \sim\left\|\nabla_{T} \varphi-\frac{3}{2} \varphi \vartheta\right\|_{-1, G}^{2}, \forall \varphi \in L^{2}(G) .
$$

With the help of Lemma 6.6 below, we conclude that (6.10) holds. 
Lemma 6.6 For all $\varphi \in L^{2}(G)$, we have

$$
\|\varphi\|_{G} \lesssim\left\|\nabla_{T} \varphi-\frac{3}{2} \varphi \vartheta\right\|_{-1, G}
$$

Proof. We first prove that

$$
\|\varphi\|_{G} \lesssim\|\varphi\|_{-1, G}+\left\|\nabla_{T} \varphi-\frac{3}{2} \varphi \vartheta\right\|_{-1, G}, \forall \varphi \in L^{2}(G)
$$

Indeed define

$$
\Sigma=\{x \in \Gamma:|x| \in(1,2)\} .
$$

Then by a result due to Nečas (see Theorem I.2.2 of [10]), we have

$$
\left\|r^{-\frac{3}{2}} \varphi\right\|_{\Sigma} \lesssim\left\|r^{-\frac{3}{2}} \varphi\right\|_{-1, \Sigma}+\left\|\nabla\left(r^{-\frac{3}{2}} \varphi\right)\right\|_{-1, \Sigma}
$$

for any $\varphi \in L^{2}(G)$. Since

$$
\nabla\left(r^{-\frac{3}{2}} \varphi\right)=r^{-\frac{5}{2}}\left(\nabla_{T} \varphi-\frac{3}{2} \varphi \vartheta\right)
$$

we deduce that

$$
\left\|r^{-\frac{3}{2}} \varphi\right\|_{\Sigma} \lesssim\left\|r^{-\frac{3}{2}} \varphi\right\|_{-1, \Sigma}+\left\|r^{-\frac{5}{2}}\left(\nabla_{T} \varphi-\frac{3}{2} \varphi \vartheta\right)\right\|_{-1, \Sigma} .
$$

Clearly we have $\left\|r^{-\frac{3}{2}} \varphi\right\|_{\Sigma} \sim\|\varphi\|_{G}$, hence (6.13) holds if we can show that

$$
\left\|r^{-\frac{3}{2}} \varphi\right\|_{-1, \Sigma} \lesssim\|\varphi\|_{-1, G}
$$

as well as

$$
\left\|r^{-\frac{5}{2}}\left(\nabla_{T} \varphi-\frac{3}{2} \varphi \vartheta\right)\right\|_{-1, \Sigma} \lesssim\left\|\nabla_{T} \varphi-\frac{3}{2} \varphi \vartheta\right\|_{-1, G} .
$$

For the first estimate we can write

$$
\begin{aligned}
\left\|r^{-\frac{3}{2}} \varphi\right\|_{-1, \Sigma} & =\sup _{\chi \in H_{0}^{1}(\Sigma)} \frac{1}{\|\nabla \chi\|_{\Sigma}} \int_{\Sigma} r^{-\frac{3}{2}} \varphi \chi d x \\
& =\sup _{\chi \in H_{0}^{1}(\Sigma)} \frac{1}{\|\nabla \chi\|_{\Sigma}} \int_{1}^{2} \int_{G} \varphi(\vartheta) \chi(r \vartheta) d \sigma(\vartheta) r^{\frac{1}{2}} d r .
\end{aligned}
$$

Hence by the definition of the norm in $H^{-1}(G)$, we get

$$
\begin{array}{r}
\left\|r^{-\frac{3}{2}} \varphi\right\|_{-1, \Sigma} \lesssim\|\varphi\|_{-1, G} \sup _{\chi \in H_{0}^{1}(\Sigma)} \frac{1}{\|\nabla \chi\|_{\Sigma}} \int_{1}^{2}\left\|\nabla_{T} \chi(r \cdot)\right\|_{G} r^{\frac{1}{2}} d r \\
\lesssim\|\varphi\|_{-1, G} \sup _{\chi \in H_{0}^{1}(\Sigma)} \frac{1}{\|\nabla \chi\|_{\Sigma}}\left(\int_{1}^{2}\left\|\nabla_{T} \chi(r \cdot)\right\|_{G}^{2} d r\right)^{\frac{1}{2}} .
\end{array}
$$

But using the identity

$$
\nabla \chi=r^{-1} \nabla_{T} \chi+\partial_{r} \chi \theta
$$


and reminding that $\nabla_{T} \chi$ is perpendicular to $\theta$, we have

$$
\|\nabla \chi\|_{\Sigma}^{2} \geq \int_{1}^{2}\left\|\nabla_{T} \chi(r \cdot)\right\|_{G}^{2} d r .
$$

This estimate in (6.17) proves (6.15).

The second assertion (6.16) follows with the same arguments componentwise.

We now prove (6.12) by a contradiction argument. Assume that it does not hold. Then there exists a sequence of functions $\varphi_{n} \in L^{2}(G), n \in \mathbb{N}^{*}$ such that

$$
\left\|\varphi_{n}\right\|_{G}=1, \forall n \in \mathbb{N}^{*}
$$

and

$$
\left\|\nabla_{T} \varphi_{n}-\frac{3}{2} \varphi_{n} \vartheta\right\|_{-1, G} \lesssim \frac{1}{n}, \forall n \in \mathbb{N}^{*}
$$

But due to the compact embedding of $L^{2}(G)$ into $H^{-1}(G)$, there exists a subsequence, still denoted by $\left(\varphi_{n}\right)_{n \in \mathbb{N}}$, and an element $\varphi \in H^{-1}(G)$ such that

$$
\varphi_{n} \rightarrow \varphi \text { in } H^{-1}(G), \text { as } n \rightarrow \infty \text {. }
$$

But this property, (6.19) and the estimate (6.13) imply that $\left(\varphi_{n}\right)_{n \in \mathbb{N}}$ is a Cauchy sequence in $L^{2}(G)$ and hence

$$
\varphi_{n} \rightarrow \varphi \text { in } L^{2}(G), \text { as } n \rightarrow \infty
$$

and by (6.18), we have

$$
\|\varphi\|_{G}=1
$$

But from (6.19) and (6.20), $\varphi$ also satisfies

$$
\nabla_{T} \varphi-\frac{3}{2} \varphi \vartheta=0
$$

This implies that $\varphi=0$ and is in contradiction with (6.21).

Corollary 6.7 There exits a discrete set $\Lambda_{0} \subset \mathbb{C} \backslash \Lambda_{\text {Dir }}(\Gamma)$ such that for all $\lambda \in \mathbb{C} \backslash\left(\Lambda_{\text {Dir }}(\Gamma) \cup \Lambda_{0}\right)$, $\mathcal{A}(\lambda)$ is an isomorphism, moreover $\mathcal{A}(\lambda)$ is meromorphic in $\mathbb{C} \backslash \Lambda_{\operatorname{Dir}}(\Gamma)$.

Proof. The two previous Lemmas show that the family $\mathcal{A}(\lambda)$ forms a holomorphic family of Fredholm operator of index zero in $U=\mathbb{C} \backslash \Lambda_{\text {Dir }}(\Gamma)$. Since there exists one $\mu \in U$ such that $\mathcal{A}(\mu)$ is an isomorphism, the result follows from the Appendix 2.2.5 of [27] (consequence of results from $[11,12,13,14])$.

We also need to introduce two subsets of $\Lambda_{0}$, namely

$$
\begin{aligned}
\Lambda_{0 g}= & \left\{\lambda \in \Lambda_{0}: \lambda+2 \notin \Lambda_{\text {Dir }}(\Gamma)\right\}, \\
\Lambda_{0 e}= & \left\{\lambda \in \Lambda_{0}: \lambda+2 \in \Lambda_{\text {Dir }}(\Gamma)\right. \text { and satisfying } \\
& \left.\exists \varphi \in \operatorname{ker} \mathcal{A}(\lambda) \backslash\{\mathbf{0}\}:\left(\vartheta \cdot c(\lambda) \mathcal{L}_{\operatorname{Dir}}(\lambda)^{-1} g(\lambda-1) \varphi, \tau\right)_{G}=0, \forall \tau \in V_{\text {Dir }}(\lambda+2)\right\} .
\end{aligned}
$$

The first case is a generic one, while the second one is an exceptional one. 
For $\lambda \in \Lambda_{\text {Dir }}(\Gamma)$, the operator $\mathcal{A}(\lambda)$ is not well-defined, hence we introduce the closed subspace $X_{\lambda}$ of $L^{2}(G)$ as follows

$$
X_{\lambda}:=\left\{\varphi \in L^{2}(G):\left(\nabla_{T} \varphi+(\lambda-1) \varphi \vartheta, \chi\right)=0, \forall \chi \in V_{\text {Dir }}(\lambda)^{3}\right\} .
$$

Then for $\varphi \in X_{\lambda}$, we define $\mathcal{L}_{\text {Dir }}(\lambda)^{-1}\left(\nabla_{T} \varphi+(\lambda-1) \varphi \vartheta\right)$ as the unique solution $\mathbf{v} \in \mathbf{H}_{0}^{1}(G)$ of

$$
\mathcal{L}(\lambda) \mathbf{v}=\nabla_{T} \varphi+(\lambda-1) \varphi \vartheta
$$

that is orthogonal (in $\mathbf{L}^{2}(G)$ ) to any elements of $V_{\text {Dir }}(\lambda)^{3}$. Obviously $\mathbf{v}+\chi$, with $\chi \in V_{\text {Dir }}(\lambda)^{3}$ is still a solution of this problem. Then we introduce the linear and continuous mapping

$$
\mathcal{B}(\lambda): X_{\lambda} \times V_{\text {Dir }}(\lambda)^{3} \rightarrow L^{2}(G):(\varphi, \chi) \rightarrow d(\lambda)\left(\mathcal{L}_{\text {Dir }}(\lambda)^{-1}\left(\nabla_{T} \varphi+(\lambda-1) \varphi \vartheta\right)+\chi\right),
$$

and the set

$$
\Lambda_{1}:=\left\{\lambda \in \Lambda_{\text {Dir }}(\Gamma): \operatorname{ker} \mathcal{B}(\lambda) \neq\{\mathbf{0}\}\right\} .
$$

As before we need to introduce two subsets of $\Lambda_{1}$, namely

$$
\begin{aligned}
& \Lambda_{1 g}=\left\{\lambda \in \Lambda_{1}: \lambda+2 \notin \Lambda_{\operatorname{Dir}}(\Gamma)\right\}, \\
& \Lambda_{1 e}=\left\{\lambda \in \Lambda_{1}: \lambda+2 \in \Lambda_{\operatorname{Dir}}(\Gamma)\right. \text { and satisfying } \\
& \left.\exists(\varphi, \chi) \in \operatorname{ker} \mathcal{B}(\lambda) \backslash\{\mathbf{0}\}:\left(\vartheta \cdot c(\lambda)\left(\mathcal{L}_{\operatorname{Dir}}(\lambda)^{-1} g(\lambda-1) \varphi+\chi\right), \tau\right)_{G}=0, \forall \tau \in V_{\operatorname{Dir}}(\lambda+2)\right\} .
\end{aligned}
$$

We are now ready to characterize the corner singularities of type 2 .

Theorem 6.8 A complex number $\lambda$, with $\Re \lambda>1 / 2$ is a corner singular exponent of type 2 if and only if $\lambda-1 \in \Lambda^{(2)}=\Lambda_{0 g} \cup \Lambda_{0 e} \cup \Lambda_{1 g} \cup \Lambda_{1 e}$.

Proof. For a singularity of type 2 , as $q=0$, by (6.2b), $\psi=r^{\lambda-1} \psi_{0}$ with $\psi_{0} \in \mathbf{L}^{2}(G)$ satisfies

$$
\Delta \operatorname{curl} \psi=0, \operatorname{div} \psi=0 \text { in } \Gamma \text {, with } \psi=0 \text { on } \partial \Gamma \text {. }
$$

This implies that curl $\psi$ is regular in $\Gamma$. As $\Delta \operatorname{curl} \psi=\operatorname{curl} \Delta \psi$, we deduce that

$$
\operatorname{curl} \Delta \psi=0 \text {. }
$$

Therefore there exists $\Phi$ such that

$$
\Delta \psi=-\nabla \Phi \text { in } \Gamma
$$

with $\Phi=\frac{1}{\lambda-2}(\Delta \psi) \cdot \mathbf{x}$ if $\lambda \neq 2$, due to (2.4), otherwise (see the identity [7, (6.11)])

$$
\Phi(r, \vartheta)=r^{-1} \varphi(\vartheta),
$$

with $\varphi \in L_{\text {loc }}^{2}(G)$. As $\Delta \psi=-\operatorname{curl} \operatorname{curl} \psi, \Phi$ is regular in $\Gamma$ and since $\Delta \psi$ belongs to $\mathbf{H}^{-1}(\Sigma)$ (recalling that $\Sigma$ was defined by (6.14)) by Corollary I.2.2 of [10], we deduce that $\Phi \in L^{2}(\Sigma)$. Hence

$$
\Phi=r^{\lambda-2} \varphi(\vartheta)
$$

with $\varphi \in L^{2}(G)$.

By (6.23) and the boundary condition $\psi=0$ on $\Gamma$, we remark that $\psi_{0}$ satisfies

$$
\mathcal{L}(\lambda-1) \psi_{0}=g(\lambda-2) \varphi \text { in } G, \quad \psi_{0}=0 \text { on } \partial G,
$$


reminding (6.6).

Now we distinguish two cases: $\lambda-1 \in \Lambda_{\text {Dir }}(\Gamma)$ or not.

1. If $\lambda-1 \notin \Lambda_{\text {Dir }}(\Gamma)$, then $\psi_{0}$ is given by

$$
\psi_{0}=\mathcal{L}_{\text {Dir }}(\lambda-1)^{-1}(g(\lambda-2) \varphi) .
$$

Now we want to take advantage of the divergence free property of $\psi$. Owing to (6.7), the constraint $\operatorname{div} \psi=0$ from (6.22) is equivalent to

$$
\mathcal{A}(\lambda-1) \varphi=0 \text { on } G \text {. }
$$

From Corollary 6.7, we conclude that $\varphi=0$ except if $\lambda-1$ is in $\Lambda_{0}$. In this second case, we find a non trivial $\varphi$ and hence a non trivial $\psi$. In the first case, $\varphi$ is zero and since $\lambda-1$ is not in $\Lambda_{\text {Dir }}(\Gamma)$, we deduce that $\psi=0$. This last case is out of interest since then we are back to singularities of type 1 .

2. If $\lambda-1 \in \Lambda_{\text {Dir }}(\Gamma)$, then a solution $\psi_{0}$ to (6.24) exists if and only if $g(\lambda-2) \varphi$ is orthogonal to any element of $V_{\text {Dir }}(\lambda-1)^{3}$, or equivalently if and only if $\varphi$ belongs to $X_{\lambda-1}$. In that situation, $\psi_{0}$ is not unique but is given by

$$
\psi_{0}=\mathcal{L}_{\text {Dir }}(\lambda-1)^{-1}(g(\lambda-2) \varphi)+\chi,
$$

for any $\chi \in V_{\operatorname{Dir}}(\lambda-1)^{3}$. The divergence free property of $\psi$ being equivalent to $d(\lambda-1) \psi_{0}=0$, we find equivalently $(\varphi, \chi) \in \operatorname{ker} \mathcal{B}(\lambda-1)$. Hence a non trivial solution $\psi_{0}$ exists if and only if this kernel is not reduced to $\mathbf{0}$, which precisely means that $\lambda-1$ has to be in $\Lambda_{1}$.

Once $\psi$ is known, it remains to find $\mathbf{u}$ solution of (6.2c) with $q=0$, namely

$$
\operatorname{curl} \mathbf{u}=\psi, \operatorname{div} \mathbf{u}=0 \text { in } \Gamma \text {, with } \mathbf{u} \times \mathbf{n}=0 \text { on } \partial \Gamma \text {. }
$$

But (2.5) yields

$$
\operatorname{curl}(\psi \times \mathbf{x})=(\lambda+1) \psi,
$$

and therefore

$$
\operatorname{curl}\left(\mathbf{u}-\frac{1}{\lambda+1} \psi \times \mathbf{x}\right)=0 .
$$

Hence there exists a scalar field $\Xi=r^{\lambda+1} \xi$ with $\xi \in H_{0}^{1}(G)$ such that

$$
\mathbf{u}-\frac{1}{\lambda+1} \psi \times \mathbf{x}=\nabla \Xi
$$

The divergence free property of $\mathbf{u}$ then gives

$$
\Delta \Xi=-\frac{1}{\lambda+1} \operatorname{div}(\psi \times \mathbf{x})=-\frac{1}{\lambda+1}(\mathbf{x} \cdot \operatorname{curl} \psi),
$$

that is equivalent to

$$
\mathcal{L}(\lambda+1) \xi=-\frac{1}{\lambda+1} h,
$$

where $h$ is given by (reminding (6.8) and the definition of $\psi_{0}$ )

$$
h=\vartheta \cdot c(\lambda-1) \psi_{0}=\vartheta \cdot c(\lambda-1)\left(\mathcal{L}_{\operatorname{Dir}}(\lambda-1)^{-1}(g(\lambda-2) \varphi)+\chi\right),
$$


with $\mathbf{0} \neq \varphi \in \operatorname{ker} \mathcal{A}(\lambda-1)$ and $\chi=0$ if $\lambda-1$ belongs to $\Lambda_{0}$ and $\mathbf{0} \neq(\varphi, \chi) \in \operatorname{ker} \mathcal{B}(\lambda-1)$ if $\lambda-1$ belongs to $\Lambda_{1}$. Therefore we again need to distinguish between the case $\lambda+1$ in $\Lambda_{\text {Dir }}(\Gamma)$ or not. In the case $\lambda+1 \notin \Lambda_{\text {Dir }}(\Gamma)$, corresponding to the case $\lambda-1 \in \Lambda_{0 g}$ or $\lambda-1 \in \Lambda_{1 g}$, there is no condition on $h$, and we find no additional condition on $\lambda$ to find $\xi$ and then $\mathbf{u}$. In the case $\lambda+1 \in \Lambda_{\text {Dir }}(\Gamma), \xi$ exists if and only if $h$ satisfies the orthogonality condition

$$
(h, \tau)=0, \forall \tau \in V_{\text {Dir }}(\lambda+1),
$$

which corresponds to the condition $\lambda-1$ in $\Lambda_{0 e}$ or $\Lambda_{1 e}$.

To describe the corner singular exponents of type 3 , we clearly need to characterize the non trivial solutions of $(6.2 \mathrm{a})$.

Lemma 6.9 A non zero solution $q=r^{\nu} Q(\vartheta)$ with $\nu \in \mathbb{C}$ and a function $Q$ defined on $G$ of

$$
\Delta^{2} q=0 \text { in } \Gamma \text {, with } q=\Delta q=0 \text { on } \partial \Gamma
$$

exists if and only if either $\nu \in \Lambda_{\text {Dir }}(\Gamma)$ or $\nu-2 \in \Lambda_{\text {Dir }}(\Gamma)$. In the first case, $Q$ belongs to $V_{\text {Dir }}(\nu)$, while in the second case $\mathcal{L}(\nu) Q$ belongs to $V_{\mathrm{Dir}}(\nu-2)$ if $\nu \neq \frac{1}{2}$.

Proof. If $q$ is a solution of (6.26), by setting $s=\Delta q$, we get the equivalent lower triangular system

$$
\begin{aligned}
& \Delta s=0 \text { in } \Gamma, \text { with } s=0 \text { on } \partial \Gamma, \\
& \Delta q=s \text { in } \Gamma, \text { with } q=0 \text { on } \partial \Gamma .
\end{aligned}
$$

Hence two types of singularities appear:

Type 1: $s=0$ and we have to find a general non-zero solution $q$ of

$$
\Delta q=0 \text { in } \Gamma \text {, with } q=0 \text { on } \partial \Gamma .
$$

Therefore $\nu$ belongs to $\Lambda_{\text {Dir }}(\Gamma)$ and $Q$ belongs to $V_{\text {Dir }}(\nu)$.

Type 2: $s$ is a general non-zero solution of (6.27a) and $q$ a particular solution of $(6.27 \mathrm{~b})$. Since $s=r^{\nu-2} S$, with a function $S$ defined in $G$, we find that $\nu-2$ belongs to $\Lambda_{\text {Dir }}(\Gamma)$ and $S \in V_{\text {Dir }}(\nu-2)$. Now $q$ is solution of $(6.27 \mathrm{~b})$ if and only if

$$
\mathcal{L}(\nu) Q=S \text { in } G, \text { with } Q=0 \text { on } \partial G .
$$

If $\nu \neq \frac{1}{2}$, a solution $Q$ of (6.28) always exists since either $\nu \notin \Lambda_{\text {Dir }}(\Gamma)$ and then $\mathcal{L}_{\text {Dir }}(\nu)$ is invertible or $\nu \in \Lambda_{\text {Dir }}(\Gamma)$ and a solution exists since $S$ is orthogonal to any element of $V_{\operatorname{Dir}}(\nu-2)$.

Notice that

$$
\mathcal{L}(\nu) S=(\mathcal{L}(\nu)-\mathcal{L}(\nu-2)) S=2(2 \nu-1) S,
$$

hence if $\nu \neq \frac{1}{2}$, a solution $Q$ of $(6.28)$ is given by $\frac{1}{2(2 \nu-1)} S$.

If $\nu=\frac{1}{2}$, as by assumption $\nu-2=-\frac{3}{2}$ belongs to $\Lambda_{\operatorname{Dir}}(\Gamma)$, then $\frac{1}{2}$ is also in $\Lambda_{\operatorname{Dir}}(\Gamma)$. Since in that case, $S$ does not satisfy the orthogonality relation, $Q$ exists in the form $r^{\frac{1}{2}}(\ln r S+\psi)$, with $\psi \in H_{0}^{1}(G)$, see Theorem 4.22 of [26]. 
Before stating our result about corner singular exponents of type 3, let us introduce the following sets:

$$
\begin{aligned}
\Lambda_{3 g}= & \left\{\lambda \in \mathbb{C}: \lambda-2 \in \Lambda_{\text {Dir }}(\Gamma) \text { and } \lambda \notin \Lambda_{\text {Dir }}(\Gamma) \cup \Lambda_{0}\right\}, \\
\Lambda_{3 e 1}= & \left\{\lambda \in \Lambda_{0}: \lambda-2 \in \Lambda_{\text {Dir }}(\Gamma) \text { such that } \exists S \in V_{\text {Dir }}(\lambda-2) \backslash\{0\}:\right. \\
& \left.\left(d(\lambda)\left(\mathcal{L}_{\text {Dir }}(\lambda)^{-1}\left(\nabla_{T} S \times \vartheta\right)\right), \chi\right)_{G}=0, \forall \chi \in \operatorname{ker} \mathcal{A}(-(\lambda+3)\}\right\}, \\
\Lambda_{3 e 2}= & \left\{\lambda \in \Lambda_{\text {Dir }}(\Gamma): \lambda-2 \in \Lambda_{\text {Dir }}(\Gamma) \text { such that } \operatorname{ker} \mathcal{C}(\lambda) \neq\{\mathbf{0}\}\right\} .
\end{aligned}
$$

In the case when $\lambda \in \Lambda_{\text {Dir }}(\Gamma)$ and $\lambda-2 \in \Lambda_{\text {Dir }}(\Gamma)$, we define the operator $\mathcal{C}(\lambda)$ as follows. Define the closed subspace $Y_{\lambda}$ of $L^{2}(G) \times V_{\text {Dir }}(\lambda-2)$ as follows

$$
Y_{\lambda}:=\left\{(\varphi, S) \in L^{2}(G) \times V_{\text {Dir }}(\lambda-2):\left(g(\lambda-1) \varphi+\nabla_{T} S \times \vartheta, \chi\right)_{G}=0, \forall \chi \in V_{\text {Dir }}(\lambda)^{3}\right\} .
$$

For an element $(\varphi, S)$ in $Y_{\lambda}$, as before we can define $\mathcal{L}_{\text {Dir }}(\lambda)^{-1}\left(g(\lambda-1) \varphi+\nabla_{T} S \times \vartheta\right)$ and introduce the linear and bounded operator

$$
\mathcal{C}(\lambda): Y_{\lambda} \times V_{\text {Dir }}(\lambda)^{3} \rightarrow L^{2}(G):(\varphi, S, \chi) \rightarrow d(\lambda)\left(\mathcal{L}_{\text {Dir }}(\lambda)^{-1}\left(g(\lambda-1) \varphi+\nabla_{T} S \times \vartheta\right)+\chi\right) .
$$

These sets will be used in the construction of $\psi$ in case of singular exponent of type 3 , for the construction of $\mathbf{u}$, we further need the following subsets:

$$
\begin{aligned}
\Lambda_{3 g, g} & =\left\{\lambda \in \Lambda_{3 g}: \lambda+2 \notin \Lambda_{\text {Dir }}(\Gamma)\right\}, \\
\Lambda_{3 e j, g} & =\left\{\lambda \in \Lambda_{3 e j}: \lambda+2 \notin \Lambda_{\text {Dir }}(\Gamma)\right\}, j \in\{1,2\} .
\end{aligned}
$$

As for singularities of type 2 , if $\lambda$ belongs to $\Lambda_{3 g}$ (resp. $\Lambda_{3 e j}$ ) is such that $\lambda+2 \in \Lambda_{\text {Dir }}(\Gamma)$, the situation is more delicate. In the first two cases, we need to define

$$
\begin{gathered}
\Lambda_{3 g, e}=\left\{\lambda \in \Lambda_{3 g}: \lambda+2 \in \Lambda_{\text {Dir }}(\Gamma) \text { satisfying (6.29) below }\right\}, \\
\Lambda_{3 e 1, e}=\left\{\lambda \in \Lambda_{3 e 1}: \lambda+2 \in \Lambda_{\text {Dir }}(\Gamma) \text { satisfying (6.29) below }\right\}, \\
\exists(\varphi, S) \in L^{2}(G) \times V_{\text {Dir }}(\lambda-2) \backslash\{\mathbf{0}\}: \\
\left(\vartheta \cdot c(\lambda) \mathcal{L}_{\text {Dir }}(\lambda)^{-1}\left(g(\lambda-1) \varphi+\nabla_{T} S \times \vartheta\right), \tau\right)_{G}=0, \forall \tau \in V_{\text {Dir }}(\lambda+2) .
\end{gathered}
$$

Finally in the last case, we define

$$
\begin{aligned}
& \Lambda_{3 e 2, e}=\left\{\lambda \in \Lambda_{3 e 2}: \lambda+2 \in \Lambda_{\text {Dir }}(\Gamma) \text { such that } \exists(\varphi, S, \chi) \in Y_{\lambda} \times V_{\text {Dir }}(\lambda)^{3} \backslash\{\mathbf{0}\}:\right. \\
& \left.\left(\vartheta \cdot c(\lambda)\left(\mathcal{L}_{\text {Dir }}(\lambda)^{-1}\left(g(\lambda-1) \varphi+\nabla_{T} S \times \vartheta\right)+\chi\right), \tau\right)_{G}=0, \forall \tau \in V_{\text {Dir }}(\lambda+2)\right\} .
\end{aligned}
$$

Here is our result about corner singular exponents of type 3 .

Theorem 6.10 A complex number $\lambda$ with $\Re \lambda>\frac{1}{2}$ is a corner singular exponent of type 3 if and only if $\lambda-1 \in \Lambda^{(3)}:=\Lambda_{\text {Dir }}(\Gamma) \cup \Lambda_{3 g, g} \cup \Lambda_{3 g, e} \cup \Lambda_{3 e 1, g} \cup \Lambda_{3 e 1, e} \cup \Lambda_{3 e 2, g} \cup \Lambda_{3 e 2, e}$.

Proof. Let $q=r^{\lambda-1} Q$ be a solution of (6.2a). Then owing to Lemma 6.9 either $\lambda-1 \in \Lambda_{\text {Dir }}(\Gamma)$ and $Q \in V_{\operatorname{Dir}}(\lambda-1)$ or $\lambda-3 \in \Lambda_{\operatorname{Dir}}(\Gamma)$ with $\Delta q=r^{\lambda-3} S$ and $S \in V_{\operatorname{Dir}}(\lambda-3)$ if $\lambda-1 \neq \frac{1}{2}$.

In the first case, $\Delta q=0$ and therefore as particular solution of (6.2b) we can chose $\psi=\mathbf{0}$. Consequently $(6.2 \mathrm{c})$ reduces to

$$
\operatorname{curl} \mathbf{u}=0, \operatorname{div} \mathbf{u}=q \text { in } \Gamma \text {, with } \mathbf{u} \times \mathbf{n}=0 \text { on } \partial \Gamma .
$$


The first condition and the boundary condition allow to write $\mathbf{u}=\nabla\left(r^{\lambda+1} \varphi\right)$, with $\varphi \in H_{0}^{1}(G)$, and the divergence constraint $\operatorname{div} \mathbf{u}=q$ becomes

$$
\mathcal{L}(\lambda+1) \varphi=Q \text { in } G .
$$

This problem has always a solution because in the case $\lambda+1 \in \Lambda_{\text {Dir }}(\Gamma), Q$ is orthogonal to any element of $V_{\text {Dir }}(\lambda+1)$.

Let us go on with the second case, namely, when $\lambda-3 \in \Lambda_{\text {Dir }}(\Gamma)$. First we notice that we can assume that $\lambda-1 \neq \frac{1}{2}$. Indeed if $\lambda-1=\frac{1}{2}$, then $\lambda-3=-\frac{3}{2}$ that by assumption belongs to $\Lambda_{\text {Dir }}(\Gamma)$, but then $\frac{1}{2}$ belongs to $\Lambda_{\operatorname{Dir}}(\Gamma)$ as well, and by the first case, we have previously shown that it generates the corner singular exponent $\frac{3}{2}$.

As $\lambda-1 \neq \frac{1}{2}$, problem $(6.2 \mathrm{~b})$ becomes

$$
\Delta \operatorname{curl} \psi=-\nabla\left(r^{\lambda-3} S\right), \operatorname{div} \psi=0 \text { in } \Gamma \text {, with } \psi=0 \text { on } \partial \Gamma,
$$

with $S \in V_{\text {Dir }}(\lambda-3)$. But due to $(2.5)$, we have

$$
\operatorname{curl}\left(\nabla\left(r^{\lambda-3} S\right) \times \mathbf{x}\right)=\left(r \partial_{r}+2\right)\left(\nabla\left(r^{\lambda-3} S\right)\right)=(\lambda-2) \nabla\left(r^{\lambda-3} S\right) .
$$

Since -1 does not belong to $\Lambda_{\text {Dir }}(\Gamma), \lambda$ cannot be equal to 2 , therefore replacing $S$ by $-(\lambda-2) S$, that is still in $V_{\operatorname{Dir}}(\lambda-3)$, we get

$$
\operatorname{curl}\left(\Delta \psi-\nabla\left(r^{\lambda-3} S\right) \times \mathbf{x}\right)=\mathbf{0} .
$$

Consequently there exists $\varphi \in L^{2}(G)$ such that

$$
\Delta \psi=\nabla\left(r^{\lambda-3} S\right) \times \mathbf{x}+\nabla\left(r^{\lambda-2} \varphi\right),
$$

or equivalently $\psi_{0} \in H_{0}^{1}(G)$ is a solution of

$$
\mathcal{L}_{\text {Dir }}(\lambda-1) \psi_{0}=\nabla_{T} S \times \vartheta+g(\lambda-2) \varphi .
$$

Again we need to distinguish between the case $\lambda-1 \in \Lambda_{\text {Dir }}(\Gamma)$ or not.

1. If $\lambda-1 \notin \Lambda_{\text {Dir }}(\Gamma)$, then $\psi_{0}$ is given by

$$
\psi_{0}=\mathcal{L}_{\text {Dir }}(\lambda-1)^{-1}\left(\nabla_{T} S \times \vartheta\right)+\mathcal{L}_{\text {Dir }}(\lambda-1)^{-1}(g(\lambda-2) \varphi) .
$$

Hence the divergence free property of $\psi$ is equivalent to

$$
d(\lambda-1) \mathcal{L}_{\text {Dir }}(\lambda-1)^{-1}\left(\nabla_{T} S \times \vartheta\right)+\mathcal{A}(\lambda-1) \varphi=0 .
$$

As a consequence, if $\lambda-1$ is not in $\Lambda_{0}$ (equivalent to $\lambda-1 \in \Lambda_{3 g}$ ), we find $\varphi$ and then $\psi$. On the contrary, $d(\lambda-1) \mathcal{L}_{\text {Dir }}(\lambda-1)^{-1}\left(\nabla_{T} S \times \vartheta\right)$ has to be in the range of $\mathcal{A}(\lambda-1)$ which precisely means that $\lambda-1$ belongs to $\Lambda_{3 e 1}$.

2. In the case when $\lambda-1 \in \Lambda_{\operatorname{Dir}}(\Gamma)$, the right-hand side of (6.31) has to be orthogonal to $V_{\text {Dir }}(\lambda-1)^{3}$, which means that $(\varphi, S)$ belongs to $Y_{\lambda-1}$. As in the previous theorem, we then get

$$
\psi_{0}=\mathcal{L}_{\text {Dir }}(\lambda-1)^{-1}\left(g(\lambda-2) \varphi+\nabla_{T} S \times \vartheta\right)+\chi,
$$

with $\chi \in V_{\text {Dir }}(\lambda-1)^{3}$ and the divergence free constraint on $\psi$ leads to the condition $\lambda-1 \in \Lambda_{3 e 2}$. 
Once we have $\psi$ in hand, we look for $\mathbf{u}$ solution of (6.2c). As in the proof of Theorem 6.8, we then get

$$
\mathbf{u}-\frac{1}{\lambda+1} \psi \times \mathbf{x}=\nabla\left(r^{\lambda+1} \xi\right)
$$

with $\xi \in H_{0}^{1}(G)$. The divergence constraint $\operatorname{div} \mathbf{u}=q$ becomes

$$
\Delta\left(r^{\lambda+1} \xi\right)=q-\frac{1}{\lambda+1} \operatorname{div}(\psi \times \mathbf{x})=q-\frac{1}{\lambda+1}(\mathbf{x} \cdot \operatorname{curl} \psi),
$$

that is equivalent to

$$
\mathcal{L}(\lambda+1) \xi=Q-\frac{1}{\lambda+1} h
$$

where, in its full generality, we have

$$
h=\vartheta \cdot c(\lambda-1)\left(\mathcal{L}_{\operatorname{Dir}}(\lambda-1)^{-1}\left(g(\lambda-2) \varphi+\nabla_{T} S \times \vartheta\right)+\chi\right) .
$$

Consequently if $\lambda+1 \notin \Lambda_{\text {Dir }}(\Gamma)$, no more constraint are needed (corresponding to the cases $\lambda-1 \in \Lambda_{3 g, g}, \lambda-1 \in \Lambda_{3 e 1, g}$ or $\left.\lambda-1 \in \Lambda_{3 e 2, g}\right)$. On the contrary if $\lambda+1 \in \Lambda_{\text {Dir }}(\Gamma), Q-\frac{1}{\lambda+1} h$ has to be orthogonal to the elements of $V_{\text {Dir }}(\lambda+1)$. But since we have assumed that $\lambda-1 \neq \frac{1}{2}, Q$ is already orthogonal to that space, therefore it remains to impose this orthogonality property on $h$, which leads to the additional constraint $\lambda-1 \in \Lambda_{3 g, e}, \lambda-1 \in \Lambda_{3 e 1, e}$ or $\lambda-1 \in \Lambda_{3 e 2, e}$.

Finally as in subsection 5.3 we have to take into account on the constraints that the singular functions have to be locally in $V$. This leads to the following set of singular exponents (see Theorems 6.8 and 6.10)

$$
\begin{aligned}
\Lambda= & \left\{\lambda \in \mathbb{R}: \lambda+1 \in \Lambda_{\text {Dir }}(\Gamma) \text { with } \lambda>-\frac{3}{2}\right\} \\
& \cup\left\{\lambda \in \mathbb{C}: \lambda-1 \in \Lambda^{(2)} \cup \Lambda^{(3)} \text { with } \Re \lambda>\frac{1}{2}\right\} .
\end{aligned}
$$

For each $\lambda \in \Lambda$, we will fix a basis $\left\{\mathbf{S}^{\lambda, p}\right\}_{p=1}^{N(\lambda)}$, with $N(\lambda) \in \mathbb{N} \backslash\{0\}$, of the set of linearly independent solutions of system (6.2).

Remark 6.11 As usual, the minimal regularity near the corner $c$ is related to the minimal value of the real part of the elements from $\Lambda$. Analytical and/or numerical results about the set $\Lambda_{\operatorname{Dir}}(\Gamma)$ are available in $[30,2,8,20,3,22]$. Similar results for the sets $\Lambda^{(2)}$ and $\Lambda^{(3)}$ merit to be studied.

\section{$7 \quad$ Some regularity results for domains with point singularities}

To end this paper we want to prove some regularity results for domains with point singularities (case 2). Note that standard localization procedures do not work in our setting since our differential operator is of order 4 and since the multiplication by a cut-off function is not stable in $V$, in the sense that if $\mathbf{u} \in V$ and $\eta$ is a smooth function, then we do not automatically have $\eta \mathbf{u} \in V$. Hence again we use global regularity results in weighted Sobolev spaces for domains with point singularities described in section 8.2 of [21]. We restrict ourselves to this case, because such results are not available for fourth order operators in polyhedral domains.

We first recall the usual weighted Sobolev spaces of Kondratiev type. For any $\ell \in \mathbb{N}$ and $\beta>0$, we set

$$
V_{\alpha}^{\ell}(\Omega)=\left\{u \in L_{\mathrm{loc}}^{2}(\Omega): r^{\alpha-\ell+|\beta|} D^{\beta} u \in L^{2}(\Omega), \forall|\beta| \leq \ell\right\},
$$


that is a Hilbert space with its natural inner product and norm $\|\cdot\|_{\ell, \alpha}$. The vectorial version will be denoted by $\mathbf{V}_{\alpha}^{\ell}(\Omega)$. We directly check that if $u \in V_{\alpha}^{\ell}(\Omega)$, then $\partial_{j} u$ belongs to $V_{\alpha}^{\ell-1}(\Omega)$, for all $j=1,2$ or 3 , with the estimate

$$
\left\|\partial_{j} u\right\|_{\ell-1, \alpha} \lesssim\|u\|_{\ell, \alpha} .
$$

Furthermore owing to Lemma 6.1 .2 of [21], if $u \in V_{\alpha}^{\ell}(\Omega)$, then its trace $\gamma u$ on $\partial \Omega$ satisfies $r^{\alpha-\left(\ell-\frac{1}{2}\right)} \gamma u \in L^{2}(\partial \Omega)$ with the estimate

$$
\left\|r^{\alpha-\left(\ell-\frac{1}{2}\right)} \gamma u\right\|_{\partial \Omega} \lesssim\|u\|_{\ell, \alpha} .
$$

Now for a corner $c$ of $\Omega$, we introduce the operator pencil $\mathcal{C}(\lambda)$ defined by

$$
\mathcal{C}(\lambda): \mathbf{H}^{4}(G) \rightarrow \mathbf{L}^{2}(G) \times R(G): \mathbf{u} \rightarrow \mathcal{C}(\lambda) \mathbf{u}=(\mathcal{L}(\lambda-2) \mathcal{L}(\lambda) \mathbf{u}, \mathcal{B}(\lambda) \mathbf{u}),
$$

where $R(G)=\mathbf{H}_{T}^{\frac{7}{2}}(\partial G) \times \mathbf{H}_{T}^{\frac{5}{2}}(\partial G) \times H^{\frac{5}{2}+\ell}(\partial G) \times H^{\frac{1}{2}+\ell}(\partial G)$, and

$$
\mathcal{B}(\lambda) \mathbf{u}=((\gamma \mathbf{u}) \times \mathbf{n}, \gamma(c(\lambda) \mathbf{u}) \times \mathbf{n}, \gamma d(\lambda) \mathbf{u}, \gamma \mathcal{L}(\lambda-2) d(\lambda) \mathbf{u})^{\top},
$$

reminding the definition (4.1) of $B$ and the relations (6.4), (6.7), (6.8) and

$$
\Delta^{2}\left(r^{\lambda} \mathbf{u}\right)=r^{\lambda-4} \mathcal{L}(\lambda-2) \mathcal{L}(\lambda) \mathbf{u}
$$

Since our starting system is elliptic, according to the considerations from section 8.2 of [21], the operator $\mathcal{C}(\lambda)$ has the following properties.

Theorem 7.1 The operator $\mathcal{C}(\lambda)$ is a Fredholm operator for all $\lambda \in \mathbb{C}$ and is an isomorphism except for a countable number of isolated points which are the corner singular exponent described in the previous section. Further in any double sector

$$
\{\lambda \in \mathbb{C}:|\Re \lambda|<\delta|\Im \lambda|\}, \delta>0,
$$

$\mathcal{C}(\lambda)$ is an isomorphism except for a finite number of points.

Now we can state our first regularity result.

Theorem 7.2 Assume that the line $\Re \lambda=\frac{1}{2}$ is free of corner singular exponents. Then the operator $A_{0}$ is an isomorphism from $\mathbf{V}_{2}^{4}(\Omega)$ into $\mathbf{V}_{2}^{0}(\Omega) \times B \mathbf{V}_{2}^{4}(\Omega)$.

Proof. Owing to Theorem 8.2.1 of [21], the operator $A_{0}$ is Fredholm from $\mathbf{V}_{2}^{4}(\Omega)$ into $\mathbf{V}_{2}^{0}(\Omega) \times$ $B \mathbf{V}_{2}^{4}(\Omega)$; its kernel consists of functions only in $\mathbf{V}_{2}^{4}(\Omega)$ and its cokernel is made of functions $(\mathbf{v}, \underline{v})$ solution of the homogeneous adjoint problem (4.10) such that $\mathbf{v} \in \mathbf{V}_{2}^{4}(\Omega)$ and $\underline{v}$ is smooth far from the corners. But owing to a local version of Lemma 4.3, if $(\mathbf{v}, \underline{v})$ is in the cokernel of $A_{0}$, $\mathbf{v}$ belongs to the kernel of $A_{0}$.

Hence the conclusion follows if we show that the kernel of $A_{0}$ (as operator from $\mathbf{V}_{2}^{4}(\Omega)$ into $\left.\mathbf{V}_{2}^{0}(\Omega) \times B \mathbf{V}_{2}^{4}(\Omega)\right)$ is reduced to zero. So let us fix $\mathbf{u} \in \mathbf{V}_{2}^{4}(\Omega)$ such that

$$
\begin{cases}\Delta^{2} \mathbf{u}=\mathbf{0} & \text { in } \Omega \\ B \mathbf{u}=\mathbf{0} & \text { on } \partial \Omega\end{cases}
$$

Since

$$
C_{0}^{\infty}(\bar{\Omega} \backslash \mathcal{C})=\left\{v \in C^{\infty}(\bar{\Omega}): v=0 \text { in a neighborhood of the corners }\right\}
$$


is dense in $V_{2}^{4}(\Omega)$, we can fix a sequence of functions $\mathbf{u}_{n} \in C_{0}^{\infty}(\bar{\Omega} \backslash \mathcal{C})^{3}, n \in \mathbb{N}$ such that

$$
\mathbf{u}_{n} \rightarrow \mathbf{u} \text { in } \mathbf{V}_{2}^{4}(\Omega) \text {, as } n \rightarrow \infty .
$$

Hence applying Green's formula (4.7) we get

$$
\begin{aligned}
& a\left(\mathbf{u}_{n}, \mathbf{u}_{n}\right)=\int_{\Omega} \Delta^{2} \mathbf{u}_{n} \cdot \overline{\mathbf{u}}_{n} \\
+ & \int_{\partial \Omega}\left(\operatorname{div} \Delta \mathbf{u}_{n} \overline{\mathbf{u}}_{n} \cdot \mathbf{n}-\Delta \mathbf{u}_{n} \cdot \mathbf{n} \operatorname{div} \overline{\mathbf{u}}_{n}-\operatorname{curl} \Delta \mathbf{u}_{n} \cdot\left(\overline{\mathbf{u}}_{n} \times \mathbf{n}\right)-\Delta \mathbf{u}_{n} \cdot\left(\operatorname{curl} \overline{\mathbf{u}}_{n} \times \mathbf{n}\right)\right) .
\end{aligned}
$$

As $\mathbf{V}_{2}^{4}(\Omega)$ is continuously embedded into $\mathbf{H}^{2}(\Omega)$, the left-hand side of this identiy tends to $a(\mathbf{u}, \mathbf{u})$ as $n$ goes to $\infty$. Hence it remains to pass to the limit in each term of the right-hand side. For the first term, we notice that (7.3) implies that

$$
\begin{gathered}
r^{-2} \mathbf{u}_{n} \rightarrow r^{-2} \mathbf{u} \text { in } \mathbf{L}^{2}(\Omega), \text { as } n \rightarrow \infty, \\
r^{2} \Delta^{2} \mathbf{u}_{n} \rightarrow r^{2} \Delta^{2} \mathbf{u} \text { in } \mathbf{L}^{2}(\Omega), \text { as } n \rightarrow \infty,
\end{gathered}
$$

and consequently, by Cauchy-Schwarz's inequality we deduce that

$$
\int_{\Omega} \Delta^{2} \mathbf{u}_{n} \cdot \overline{\mathbf{u}}_{n} \rightarrow \int_{\Omega} r^{2} \Delta^{2} \mathbf{u} \cdot\left(r^{-2} \overline{\mathbf{u}}\right)=0, \text { as } n \rightarrow \infty .
$$

Similarly, using (7.1) and (7.2), (7.3) implies that

$$
\begin{array}{r}
r^{-\frac{3}{2}} \mathbf{u}_{n} \rightarrow r^{-\frac{3}{2}} \mathbf{u} \text { in } \mathbf{L}^{2}(\partial \Omega), \text { as } n \rightarrow \infty, \\
r^{\frac{3}{2}} \operatorname{div} \Delta \mathbf{u}_{n} \rightarrow r^{\frac{3}{2}} \operatorname{div} \Delta \mathbf{u} \text { in } \mathbf{L}^{2}(\partial \Omega), \text { as } n \rightarrow \infty,
\end{array}
$$

and as before we deduce that

$$
\int_{\partial \Omega} \operatorname{div} \Delta \mathbf{u}_{n} \overline{\mathbf{u}}_{n} \cdot \mathbf{n} \rightarrow \int_{\partial \Omega} \operatorname{div} \Delta \mathbf{u} \overline{\mathbf{u}} \cdot \mathbf{n}=0, \text { as } n \rightarrow \infty .
$$

The same argument applies to the other boundary terms and yields

$\int_{\partial \Omega}\left(\operatorname{div} \Delta \mathbf{u}_{n} \overline{\mathbf{u}}_{n} \cdot \mathbf{n}-\Delta \mathbf{u}_{n} \cdot \mathbf{n} \operatorname{div} \overline{\mathbf{u}}_{n}-\operatorname{curl} \Delta \mathbf{u}_{n} \cdot\left(\overline{\mathbf{u}}_{n} \times \mathbf{n}\right)-\Delta \mathbf{u}_{n} \cdot\left(\operatorname{curl} \overline{\mathbf{u}}_{n} \times \mathbf{n}\right)\right) \rightarrow 0$, as $n \rightarrow \infty$.

This means that we have shown that

$$
a(\mathbf{u}, \mathbf{u})=0,
$$

and since $\mathbf{u}$ belongs to $V$, we deduce that $\mathbf{u}=\mathbf{0}$.

With this result in hand we can use the comparison Theorem 8.2.2 of [21] that directly leads to the

Theorem 7.3 Let $\ell \in \mathbb{N}$ and $\beta \in \mathbb{R}$ such that $\beta-\ell<2$. Assume that the lines $\Re \lambda=\frac{1}{2}$ and $\Re \lambda=\ell-\beta+\frac{5}{2}$ are free of corner singular exponents. Let $\mathbf{u}_{0} \in \mathbf{V}_{2}^{4}(\Omega)$ be the unique solution of

$$
\begin{cases}\Delta^{2} \mathbf{u}_{0}=\mathbf{f} & \text { in } \Omega \\ B \mathbf{u}_{0}=\mathbf{g} & \text { on } \partial \Omega\end{cases}
$$


with $\mathbf{f} \in \mathbf{V}_{\beta}^{\ell}(\Omega)$ and $\mathbf{g} \in B \mathbf{V}_{\beta}^{\ell+4}(\Omega)$. Then $\mathbf{u}_{0}$ admits the decomposition

$$
\mathbf{u}_{0}=\mathbf{u}_{R}+\sum_{c \in \mathcal{C}} \sum_{\lambda \in \Lambda_{c}: \frac{1}{2}<\Re \lambda<\ell-\beta+\frac{5}{2}} \sum_{p} k_{c, \lambda, p} \mathbf{S}^{\lambda, p},
$$

where $\mathbf{u}_{R} \in \mathbf{V}_{\beta}^{\ell+4}(\Omega)$ and for all $c \in \mathcal{C}, \lambda \in \Lambda_{c}: \frac{1}{2}<\Re \lambda<\ell-\beta+\frac{5}{2}$ and $p, k_{c, \lambda, p} \in \mathbb{C}$.

We now exploit this result to get a decomposition of the variational solution $\mathbf{u} \in V$ of (3.9) (with $\mathbf{f} \in \mathbf{L}^{2}(\Omega)$ ) into a regular part and a singular one. Before we need a variant of Lemma 3.6 where the regular and the singular parts are orthogonal for the inner product induced by the sesquilinear form $a$. In a first step as $\eta_{c} \nabla u_{\text {Dir }}^{\lambda+1, p}$ are not in $V$, we need to correct them.

Lemma 7.4 For all $c \in \mathcal{C}, \lambda \in\left(-\frac{3}{2}, \frac{1}{2}\right): \lambda+1 \in \Lambda_{\text {Dir }}\left(\Gamma_{c}\right)$ and all $p$, there exists $\mathbf{r}_{c, \lambda, p} \in \mathbf{V}_{0}^{2}(\Omega)$ such that

$$
\mathbf{U}_{c, \lambda, p}=\eta_{c} \nabla u_{\text {Dir }}^{\lambda+1, p}-\mathbf{r}_{c, \lambda, p}
$$

belongs to $V$.

Proof. For shortness, set $\mathbf{S}=\eta_{c} \nabla u_{\mathrm{Dir}}^{\lambda+1, p}$ and drop the indices $c, \lambda$ and $p$. As $\eta$ is a radial function, we see that

$$
\begin{aligned}
\mathbf{S} \times \mathbf{n} & =\mathbf{0} \text { on } \partial \Omega \\
\operatorname{div} \mathbf{S} & =0 \text { on } \partial \Omega .
\end{aligned}
$$

On the contrary, ( curl $\mathbf{S}) \times \mathbf{n}$ is not zero on the boundary because (2.3) yields

$$
\operatorname{curl} \mathbf{S}=\nabla \eta \times \nabla u_{\text {Dir }} .
$$

But as $\nabla \eta$ is zero near $c$ and far from $c$ and as $u_{\text {Dir }}$ is smooth far from $c$, we deduce that curl $\mathbf{S}$ belongs to $C_{0}^{\infty}(\bar{\Omega} \backslash \mathcal{C})^{3}$.

Therefore we look for $\mathbf{r} \in \mathbf{V}_{0}^{2}(\Omega)$ such that

$$
\begin{cases}\mathbf{r}=\mathbf{0} & \text { on } \partial \Omega, \\ \operatorname{div} \mathbf{r}=0 & \text { on } \partial \Omega, \\ \operatorname{curl} \mathbf{r} \times \mathbf{n}=(\operatorname{curl} \mathbf{S}) \times \mathbf{n} & \text { on } \partial \Omega .\end{cases}
$$

Indeed by the expressions (4.3) and (4.4), this system is equivalent to

$$
\begin{cases}\mathbf{r}=\mathbf{0} & \text { on } \partial \Omega, \\ \mathbf{n} \cdot \partial_{n} \mathbf{r}=0 & \text { on } \partial \Omega, \\ \left(\mathbf{n} \times \partial_{n} \mathbf{r}\right) \times \mathbf{n}=(\operatorname{curl} \mathbf{S}) \times \mathbf{n} & \text { on } \partial \Omega\end{cases}
$$

This means that it suffices to require that

$$
\begin{cases}\mathbf{r}=\mathbf{0} & \text { on } \partial \Omega \\ \partial_{n} \mathbf{r}=(\operatorname{curl} \mathbf{S}) \times \mathbf{n} & \text { on } \partial \Omega .\end{cases}
$$

By the property curl $\mathbf{S} \in C_{0}^{\infty}(\bar{\Omega} \backslash \mathcal{C})^{3}$, the existence of $\mathbf{r} \in \mathbf{V}_{0}^{2}(\Omega)$ satisfying the two boundary conditions (7.8) follows. Indeed applying Theorem 1.5.1.2 of [16] in a smooth domain $\tilde{\Omega}$ that coincides with $\Omega$ except in a small neighborhood of the corners we get a function $\widetilde{\mathbf{r}} \in \mathbf{H}^{2}(\tilde{\Omega})$ 
satisfying (7.8) on the boundary of $\tilde{\Omega}$. We get the desired function by multiplying $\widetilde{\mathbf{r}}$ by a cut-off function that is equal to 1 on the support of curl $\mathbf{S}$ and equal to 0 near the corners.

Now we notice that the estimate (3.15) implies that $\mathbf{H}^{2}(\Omega) \cap V$ is a closed subspace of $V$, hence we can define the projection $P$ on $\mathbf{H}^{2}(\Omega) \cap V$ with respect to the inner product $a$. Let us further set $K=(I-P) V$.

Lemma 7.5 Under the assumptions of Lemma 3.6, any $\mathbf{u} \in V$ admits the decomposition (3.11) where $\mathbf{u}_{\mathrm{reg}} \in \mathbf{H}^{2}(\Omega) \cap V$, and $\mathbf{u}_{\text {sing }} \in K$ is given by

$$
\mathbf{u}_{\text {sing }}=\sum_{c \in \mathcal{C}} \sum_{\lambda \in\left(-\frac{3}{2}, \frac{1}{2}\right): \lambda+1 \in \Lambda_{\operatorname{Dir}}\left(\Gamma_{c}\right)} \sum_{p} d_{c, \lambda, p}(I-P) \mathbf{U}_{c, \lambda, p},
$$

where for $\lambda \in\left(-\frac{3}{2}, \frac{1}{2}\right)$ such that $\lambda+1 \in \Lambda_{\text {Dir }}\left(\Gamma_{c}\right), d_{c, \lambda, p} \in \mathbb{C}$. Consequently $\mathbf{u}_{\mathrm{reg}}$ and $\mathbf{u}_{\text {sing }}$ are orthogonal, namely

$$
a\left(\mathbf{u}_{\mathrm{reg}}, \mathbf{u}_{\mathrm{sing}}\right)=0 .
$$

Proof. Let $\mathbf{u}$ be fixed in $V$. According to Lemma $3.6 \mathbf{u}$ admits the decomposition

$$
\mathbf{u}=\mathbf{u}_{\mathrm{reg}}+\sum_{c \in \mathcal{C}} \eta_{c} \sum_{\lambda \in\left(-\frac{3}{2}, \frac{1}{2}\right): \lambda+1 \in \Lambda_{\mathrm{Dir}}\left(\Gamma_{c}\right)} \sum_{p} d_{c, \lambda, p} \nabla u_{\mathrm{Dir}}^{\lambda+1, p},
$$

with $\mathbf{u}_{\mathrm{reg}} \in \mathbf{H}^{2}(\Omega)$ and $d_{c, \lambda, p}$ in $\mathbb{C}$. Hence by the previous Lemma, we have

$$
\mathbf{u}=\mathbf{u}_{\mathrm{reg}}^{(1)}+\sum_{c \in \mathcal{C}} \sum_{\lambda \in\left(-\frac{3}{2}, \frac{1}{2}\right): \lambda+1 \in \Lambda_{\mathrm{Dir}}\left(\Gamma_{c}\right)} \sum_{p} d_{c, \lambda, p} \mathbf{U}_{c, \lambda, p}
$$

with

$$
\mathbf{u}_{\mathrm{reg}}^{(1)}=\mathbf{u}_{\mathrm{reg}}+\sum_{c \in \mathcal{C}} \sum_{\lambda \in\left(-\frac{3}{2}, \frac{1}{2}\right): \lambda+1 \in \Lambda_{\mathrm{Dir}}\left(\Gamma_{c}\right)} \sum_{p} d_{c, \lambda, p} \mathbf{r}_{c, \lambda, p}
$$

that clearly belongs to $\mathbf{H}^{2}(\Omega)$. But it also belongs to $V$ because $\mathbf{u}$ is in $V$ as well as all $\mathbf{U}_{c, \lambda, p}$. We get the orthogonal decomposition by splitting $\mathbf{U}_{c, \lambda, p}$ into the sum of $P \mathbf{U}_{c, \lambda, p}$ and of $(I-P) \mathbf{U}_{c, \lambda, p}$.

We are now ready to state the main result of this section.

Theorem 7.6 Let $\ell \in \mathbb{N}$ and $\beta \in \mathbb{R}$ such that $\beta-\ell \leq 0$. Assume that the lines $\Re \lambda=\frac{1}{2}$ and $\Re \lambda=\ell-\beta+\frac{5}{2}$ are free of corner singular exponents. Assume further that $\frac{1}{2} \notin \Lambda_{N e u}\left(\Gamma_{c}\right)$, for all $c \in \mathcal{C}$. Let $\mathbf{u} \in V$ be the unique solution of (3.3) with $\mathbf{f} \in \mathbf{V}_{\beta}^{\ell}(\Omega)$. Then it admits the next decomposition

$$
\begin{aligned}
\mathbf{u} & =\mathbf{u}_{R}+\sum_{c \in \mathcal{C}}\left(\sum_{\lambda \in \Lambda_{c}: \frac{1}{2}<\Re \lambda<\ell-\beta+\frac{5}{2}} \sum_{p} k_{c, \lambda, p} \mathbf{S}^{\lambda, p}\right. \\
& \left.+\sum_{\lambda \in\left(-\frac{3}{2}, \frac{1}{2}\right): \lambda+1 \in \Lambda_{\operatorname{Dir}}\left(\Gamma_{c}\right)} \sum_{p} d_{c, \lambda, p}(I-P) \mathbf{U}_{c, \lambda, p}\right),
\end{aligned}
$$

where $\mathbf{u}_{R} \in \mathbf{V}_{\beta}^{\ell+4}(\Omega), k_{c, \lambda, p} \in \mathbb{C}$ and $d_{c, \lambda, p} \in \mathbb{C}$. 
Proof. The assumption $\beta-\ell \leq 0$ implies that $\mathbf{V}_{\beta}^{\ell}(\Omega)$ is embedded into $\mathbf{L}^{2}(\Omega)$, therefore problem (3.3) has indeed a unique solution $\mathbf{u} \in V$. Then according to Lemma 7.5, $\mathbf{u}$ admits the decomposition (3.11) where $\mathbf{u}_{\text {reg }} \in \mathbf{H}^{2}(\Omega) \cap V$, and $\mathbf{u}_{\text {sing }} \in K$ (given by (7.9)). Using a similar decomposition for $\mathbf{v}=\mathbf{v}_{\text {reg }}+\mathbf{v}_{\text {sing }}$ with $\mathbf{v}_{\text {reg }} \in \mathbf{H}^{2}(\Omega) \cap V$ and $\mathbf{v}_{\text {sing }} \in K$, (3.3) is equivalent to

$$
a\left(\mathbf{u}_{\text {reg }}, \mathbf{v}_{\text {reg }}\right)=\int_{\Omega} \mathbf{f} \cdot \overline{\mathbf{v}}_{\text {reg }}, \forall \mathbf{v}_{\text {reg }} \in \mathbf{H}^{2}(\Omega) \cap V,
$$

and the finite linear system

$$
a\left(\mathbf{u}_{\text {sing }}, \mathbf{v}_{\text {sing }}\right)=\int_{\Omega} \mathbf{f} \cdot \overline{\mathbf{v}}_{\text {sing }}, \forall \mathbf{v}_{\text {sing }} \in K .
$$

Now by Theorem 7.3 , there exists a unique solution $\mathbf{u}_{0} \in \mathbf{V}_{2}^{4}(\Omega)$ of

$$
\begin{cases}\Delta^{2} \mathbf{u}_{0}=\mathbf{f} & \text { in } \Omega \\ B \mathbf{u}_{0}=\mathbf{0} & \text { on } \partial \Omega\end{cases}
$$

that admits the decomposition (7.6) with $\mathbf{u}_{R} \in \mathbf{V}_{\beta}^{\ell+4}(\Omega)$ and $k_{c, \lambda, p} \in \mathbb{C}$.

As $\mathbf{u}_{0}$ belongs to $\mathbf{H}^{2}(\Omega)$ and satisfies $B \mathbf{u}_{0}=\mathbf{0}$ on $\partial \Omega$, its belongs to $\mathbf{H}^{2}(\Omega) \cap V$. Hence it remains to show that

$$
a\left(\mathbf{u}_{0}, \mathbf{v}_{\text {reg }}\right)=\int_{\Omega} \mathbf{f} \cdot \mathbf{v}_{\text {reg }}, \forall \mathbf{v}_{\text {reg }} \in \mathbf{H}^{2}(\Omega) \cap V .
$$

Indeed since the solution of (7.11) is unique, we will deduce that $\mathbf{u}_{0}=\mathbf{u}_{\text {reg }}$, whence the decomposition (7.10) for $\mathbf{u}$.

For an arbitrary $\mathbf{v}_{\text {reg }} \in \mathbf{H}^{2}(\Omega) \cap V$, we transform the left-hand side of (7.14) by using the decomposition (7.6). First for the term $a\left(\mathbf{u}_{R}, \mathbf{v}_{\text {reg }}\right)$, as $\mathbf{u}_{R}$ belongs to $\mathbf{H}^{4}(\Omega)$, we can apply Green's formula (4.7) to get

$$
\begin{aligned}
& a\left(\mathbf{u}_{R}, \mathbf{v}_{\text {reg }}\right)=\int_{\Omega} \Delta^{2} \mathbf{u}_{R} \cdot \overline{\mathbf{v}}_{\text {reg }} \\
& +\int_{\partial \Omega}\left(\operatorname{div} \Delta \mathbf{u}_{R} \overline{\mathbf{v}}_{\text {reg }} \cdot \mathbf{n}-\Delta \mathbf{u}_{R} \cdot \mathbf{n} \operatorname{div} \overline{\mathbf{v}}_{\text {reg }}-\operatorname{curl} \Delta \mathbf{u}_{R} \cdot\left(\overline{\mathbf{v}}_{\text {reg }} \times \mathbf{n}\right)-\Delta \mathbf{u}_{R} \cdot\left(\operatorname{curl} \overline{\mathbf{v}}_{\text {reg }} \times \mathbf{n}\right)\right) .
\end{aligned}
$$

For the term $a\left(\mathbf{S}^{\lambda, p}, \mathbf{v}_{\text {reg }}\right)$, for one $\lambda \in \Lambda_{c}$ and $p$, we denote by $\mathbf{h}_{\lambda, p}=-\Delta \mathbf{S}^{\lambda, p}$, and recall that $\mathbf{h}_{\lambda, p}$ is in $\mathbf{H}^{2}$ far from $c$ and $\Delta \mathbf{h}_{\lambda, p}=-\Delta^{2} \mathbf{S}^{\lambda, p}$ is zero near the corner $c$. Consequently one has

$$
\int_{\Omega}\left(\operatorname{curl}^{2}-\nabla \operatorname{div}\right) \mathbf{h}_{\lambda, p} \cdot \mathbf{v}_{\text {reg }}=\lim _{\varepsilon \rightarrow 0} \int_{\Omega_{\varepsilon}}\left(\operatorname{curl}^{2}-\nabla \operatorname{div}\right) \mathbf{h}_{\lambda, p} \cdot \mathbf{v}_{\text {reg }},
$$

where $\Omega_{\varepsilon}=\{\mathbf{x} \in \Omega: r(\mathbf{x})>\varepsilon\}$. Now applying Green's formula in $\Omega_{\varepsilon}$, we find that

$$
\begin{aligned}
\int_{\Omega}\left(\operatorname{curl}^{2}-\nabla \operatorname{div}\right) \mathbf{h}_{\lambda, p} \cdot \mathbf{v}_{\text {reg }} & =\lim _{\varepsilon \rightarrow 0}\left(\int_{\Omega_{\varepsilon}}\left(\operatorname{curl} \mathbf{h}_{\lambda, p} \cdot \operatorname{curl} \mathbf{v}_{\text {reg }}+\operatorname{div} \mathbf{h}_{\lambda, p} \operatorname{div} \mathbf{v}_{\text {reg }}\right)\right. \\
& \left.+\int_{\partial \Omega_{\varepsilon}}\left(\operatorname{curl} \mathbf{h}_{\lambda, p} \cdot\left(\mathbf{v}_{\text {reg }} \times \mathbf{n}\right)-\operatorname{div} \mathbf{h}_{\lambda, p} \mathbf{v}_{\text {reg }} \cdot \mathbf{n}\right)\right) .
\end{aligned}
$$

But $\mathbf{v}_{\text {reg }}$ being in $\mathbf{H}^{2}(\Omega)$ by the Sobolev embedding theorem, $\mathbf{v}_{\text {reg }}$ belongs to $C^{0, \frac{1}{2}}(\bar{\Omega})$ and since $\mathbf{v}_{\text {reg }} \times \mathbf{n}=\mathbf{0}$, we deduce that $\mathbf{v}_{\text {reg }}(c)=\mathbf{0}$ and

$$
\left|\mathbf{v}_{\text {reg }}(\mathbf{x})\right| \lesssim r^{\frac{1}{2}}
$$


Since curl $\mathbf{h}_{\lambda, p}$ and div $\mathbf{h}_{\lambda, p}$ behaves like $r^{\lambda-3}$ near $c$, we deduce that

$$
\left|\int_{\partial \Omega_{\varepsilon} \cap\{r=\varepsilon\}}\left(\operatorname{curl} \mathbf{h}_{\lambda, p} \cdot\left(\mathbf{v}_{\text {reg }} \times \mathbf{n}\right)-\operatorname{div} \mathbf{h}_{\lambda, p} \mathbf{v}_{\text {reg }} \cdot \mathbf{n}\right)\right| \lesssim \varepsilon^{\Re \lambda-\frac{1}{2}},
$$

and since $\Re \lambda-\frac{1}{2}>0$, we get

$$
\lim _{\varepsilon \rightarrow 0} \int_{\partial \Omega_{\varepsilon} \cap\{r=\varepsilon\}}\left(\operatorname{curl} \mathbf{h}_{\lambda, p} \cdot\left(\mathbf{v}_{\mathrm{reg}} \times \mathbf{n}\right)-\operatorname{div} \mathbf{h}_{\lambda, p} \mathbf{v}_{\mathrm{reg}} \cdot \mathbf{n}\right)=0 .
$$

Further as $\operatorname{div} \mathbf{h}_{\lambda, p}=-\operatorname{div} \Delta \mathbf{S}^{\lambda, p}$ is zero on $V \cap \partial \Omega$ with a sufficiently small neighborhood $V$ of the corners, $\mathbf{v}_{\text {reg }} \times \mathbf{n}=\mathbf{0}$ on $\partial \Omega$ and with the previous property, we deduce that

$$
\lim _{\varepsilon \rightarrow 0} \int_{\partial \Omega_{\varepsilon}}\left(\operatorname{curl} \mathbf{h}_{\lambda, p} \cdot\left(\mathbf{v}_{\text {reg }} \times \mathbf{n}\right)-\operatorname{div} \mathbf{h}_{\lambda, p} \mathbf{v}_{\text {reg }} \cdot \mathbf{n}\right)=-\int_{\partial \Omega} \operatorname{div} \mathbf{h}_{\lambda, p} \mathbf{v}_{\text {reg }} \cdot \mathbf{n} .
$$

On the other hand as $\operatorname{div} \mathbf{v}_{\text {reg }} \in H_{0}^{1}(\Omega)$ and curl $\mathbf{v}_{\text {reg }} \in H_{0}^{1}(\Omega)^{3}$, by Hardy's inequality we deduce

$$
r^{-1} \operatorname{div} \mathbf{v}_{\text {reg }} \in L^{2}(\Omega) \text { and } r^{-1} \operatorname{curl} \mathbf{v}_{\text {reg }} \in \mathbf{L}^{2}(\Omega) .
$$

This implies that

$$
\lim _{\varepsilon \rightarrow 0} \int_{\Omega_{\varepsilon}}\left(\operatorname{curl} \mathbf{h}_{\lambda, p} \cdot \operatorname{curl} \mathbf{v}_{\text {reg }}+\operatorname{div} \mathbf{h}_{\lambda, p} \operatorname{div} \mathbf{v}_{\text {reg }}\right)=\int_{\Omega}\left(\operatorname{curl} \mathbf{h}_{\lambda, p} \cdot \operatorname{curl} \mathbf{v}_{\text {reg }}+\operatorname{div} \mathbf{h}_{\lambda, p} \operatorname{div} \mathbf{v}_{\text {reg }}\right),
$$

and therefore we have proved that

$$
\begin{aligned}
\int_{\Omega}\left(\operatorname{curl}^{2}-\nabla \operatorname{div}\right) \mathbf{h}_{\lambda, p} \cdot \mathbf{v}_{\text {reg }} & =\int_{\Omega}\left(\operatorname{curl} \mathbf{h}_{\lambda, p} \cdot \operatorname{curl} \mathbf{v}_{\text {reg }}+\operatorname{div} \mathbf{h}_{\lambda, p} \operatorname{div} \mathbf{v}_{\text {reg }}\right) \\
& -\int_{\partial \Omega} \operatorname{div} \mathbf{h}_{\lambda, p} \mathbf{v}_{\text {reg }} \cdot \mathbf{n} .
\end{aligned}
$$

At this stage we again have to integrate by parts in the first term of this right-hand side. For that purpose, we fix a cut-off function $\eta$ with a support included in $V$ and such that $\eta=1$ near the corners. The previous considerations show that $\eta \operatorname{div} \mathbf{v}_{\text {reg }} \in H_{0}^{1}(\Omega)$ and $\eta \operatorname{curl} \mathbf{v}_{\text {reg }} \in H_{0}^{1}(\Omega)^{3}$, since $\mathcal{D}(\Omega)$ is dense in $H_{0}^{1}(\Omega)$, there exists a sequence $d_{n} \in \mathcal{D}(\Omega)$ (resp. $\left.\mathbf{w}_{n} \in \mathcal{D}(\Omega)^{3}\right), n \in \mathbb{N}$ such that

$$
\begin{gathered}
d_{n} \rightarrow \eta \text { div } \mathbf{v}_{\text {reg }} \text { in } H_{0}^{1}(\Omega), \text { as } n \rightarrow \infty, \\
\mathbf{w}_{n} \rightarrow \eta \text { curl } \mathbf{v}_{\text {reg }} \text { in } H_{0}^{1}(\Omega)^{3}, \text { as } n \rightarrow \infty .
\end{gathered}
$$

Hence

$$
\int_{\Omega}\left(\operatorname{curl} \mathbf{h}_{\lambda, p} \cdot\left(\eta \operatorname{curl} \mathbf{v}_{\mathrm{reg}}\right)+\operatorname{div} \mathbf{h}_{\lambda, p}\left(\eta \operatorname{div} \mathbf{v}_{\mathrm{reg}}\right)\right)=\lim _{n \rightarrow \infty} \int_{\Omega}\left(\operatorname{curl} \mathbf{h}_{\lambda, p} \cdot \mathbf{w}_{n}+\operatorname{div} \mathbf{h}_{\lambda, p} d_{n}\right),
$$

and by Green's formula, we deduce that

$$
\int_{\Omega}\left(\operatorname{curl} \mathbf{h}_{\lambda, p} \cdot\left(\eta \operatorname{curl} \mathbf{v}_{\text {reg }}\right)+\operatorname{div} \mathbf{h}_{\lambda, p}\left(\eta \operatorname{div} \mathbf{v}_{\text {reg }}\right)\right)=\lim _{n \rightarrow \infty} \int_{\Omega} \mathbf{h}_{\lambda, p} \cdot\left(\operatorname{curl} \mathbf{w}_{n}-\nabla d_{n}\right) .
$$


As $\mathbf{h}_{\lambda, p}$ belongs to $\mathbf{L}^{2}(\Omega)$, we can pass to the limit in this right-hand side and deduce that

$$
\begin{array}{r}
\int_{\Omega}\left(\operatorname{curl} \mathbf{h}_{\lambda, p} \cdot\left(\eta \operatorname{curl} \mathbf{v}_{\text {reg }}\right)+\operatorname{div} \mathbf{h}_{\lambda, p}\left(\eta \operatorname{div} \mathbf{v}_{\text {reg }}\right)\right) \\
=\int_{\Omega} \mathbf{h}_{\lambda, p} \cdot\left(\operatorname{curl}\left(\eta \operatorname{curl} \mathbf{v}_{\text {reg }}\right)-\nabla\left(\eta \operatorname{div} \mathbf{v}_{\text {reg }}\right)\right) .
\end{array}
$$

As $(1-\eta) \operatorname{div} \mathbf{v}_{\text {reg }}$ and $(1-\eta)$ curl $\mathbf{v}_{\text {reg }}$ are in $H^{1}$ and is zero near the corners, we can directly apply Green's formula to get

$$
\begin{aligned}
& \int_{\Omega}\left(\operatorname{curl} \mathbf{h}_{\lambda, p} \cdot\left((1-\eta) \operatorname{curl} \mathbf{v}_{\text {reg }}\right)+\operatorname{div} \mathbf{h}_{\lambda, p}\left((1-\eta) \operatorname{div} \mathbf{v}_{\text {reg }}\right)\right) \\
& \quad=\int_{\Omega} \mathbf{h}_{\lambda, p} \cdot\left(\operatorname{curl}\left((1-\eta) \operatorname{curl} \mathbf{v}_{\text {reg }}\right)-\nabla\left((1-\eta) \operatorname{div} \mathbf{v}_{\text {reg }}\right)\right) .
\end{aligned}
$$

This identity with (7.17) implies that

$$
\left.\int_{\Omega}\left(\operatorname{curl} \mathbf{h}_{\lambda, p} \cdot \operatorname{curl} \mathbf{v}_{\text {reg }}+\operatorname{div} \mathbf{h}_{\lambda, p} \operatorname{div} \mathbf{v}_{\text {reg }}\right)=\int_{\Omega} \mathbf{h}_{\lambda, p} \cdot\left(\operatorname{curl} \operatorname{curl} \mathbf{v}_{\text {reg }}\right)-\nabla \operatorname{div} \mathbf{v}_{\text {reg }}\right) .
$$

Inserting this identity in (7.16), we have shown that

$$
\int_{\Omega}\left(\operatorname{curl}^{2}-\nabla \operatorname{div}\right) \mathbf{h}_{\lambda, p} \cdot \mathbf{v}_{\text {reg }}=a\left(\mathbf{S}^{\lambda, p}, \mathbf{v}_{\text {reg }}\right)-\int_{\partial \Omega} \operatorname{div} \mathbf{h}_{\lambda, p} \mathbf{v}_{\text {reg }} \cdot \mathbf{n} .
$$

This identity with (7.15) and the expression (7.6) lead to

$$
\begin{aligned}
a\left(\mathbf{u}_{0}, \mathbf{v}_{\text {reg }}\right) & =\int_{\Omega} \Delta^{2} \mathbf{u}_{0} \cdot \overline{\mathbf{v}}_{\text {reg }}+\int_{\partial \Omega} \operatorname{div} \Delta \mathbf{u}_{0} \overline{\mathbf{v}}_{\text {reg }} \cdot \mathbf{n} \\
& =\int_{\Omega} \mathbf{f} \cdot \overline{\mathbf{v}}_{\text {reg }},
\end{aligned}
$$

due to (7.13). This proves (7.14).

\section{References}

[1] C. Amrouche, C. Bernardi, M. Dauge, and V. Girault. Vector potentials in three-dimensional nonsmooth domains. Math. Meth. Appl. Sci., 21:823-864, 1998.

[2] A. E. Beagles and J. R. Whiteman. Treatment of a re-entrant vertex in a three-dimensional poisson problem. In [17], pages 19-27. Springer-Verlag, 1985.

[3] A. E. Beagles and J. R. Whiteman. General conical singularities in threedimensional poisson problems. Math. Meth. Appl. Sci., 11:215-235, 1989.

[4] D. Boffi, F. Brezzi, and M. Fortin. Mixed finite element methods and applications, volume 44 of Springer Series in Computational Mathematics. Springer, Heidelberg, 2013.

[5] H. Brezis. Functional analysis, Sobolev spaces and partial differential equations. Universitext. Springer, New York, 2011. 
[6] F. Cakoni and H. Haddar. A variational approach for the solution of the electromagnetic interior transmission problem for anisotropic media. Inverse Probl. Imaging, 1(3):443-456, 2007.

[7] M. Costabel and M. Dauge. Singularities of electromagnetic fields in polyhedral domains. Arch. Rational Mech. Anal., 151:221-276, 2000.

[8] M. Dauge. Elliptic boundary value problems on corner domains, volume 1341 of Lecture Notes in Mathematics. Springer-Verlag, Berlin, 1988.

[9] M. Dauge. Stationary Stokes and Navier-Stokes systems on two- or three-dimensional domains with corners. I. Linearized equations. SIAM J. Math. Anal., 20(1):74-97, 1989.

[10] V. Girault and P.-A. Raviart. Finite element methods for Navier-Stokes equations, Theory and algorithms, volume 5 of Springer Series in Computational Mathematics. Springer, Berlin, 1986.

[11] B. Gramsch. Über analytische Operatorfunktionen und Indexberechnung. Studia Math., 38:313-317. (errata insert), 1970.

[12] B. Gramsch. Inversion von Fredholmfunktionen bei stetiger und holomorpher Abhängigkeit von Parametern. Math. Ann., 214:95-147, 1975.

[13] B. Gramsch and W. Kaballo. Regularisierung von Fredholmfunktionen. Math. Ann., 232(2):151-162, 1978.

[14] B. Gramsch and W. Kaballo. Spectral theory for Fredholm functions. In Functional analysis: surveys and recent results, II (Proc. Second Conf. Functional Anal., Univ. Paderborn, Paderborn, 1979), volume 68 of Notas Mat., pages 319-342. North-Holland, Amsterdam-New York, 1980.

[15] P. Grisvard. Théorèmes de traces relatifs à un polyèdre. C. R. Acad. Sci. Paris Sér. A, 278:1581-1583, 1974.

[16] P. Grisvard. Elliptic Problems in Nonsmooth Domains. Pitman, Boston-London-Melbourne, 1985.

[17] P. Grisvard, W. Wendland, and J. R. Whiteman, editors. Singularities and constructive methods of their treatment. Lecture Notes in Mathematics, vol. 1121. Springer-Verlag, BerlinHeidelberg-New York-Tokyo, 1985.

[18] Q. Hong, J. Hu, S. Shu, and J. Xu. A discontinuous Galerkin method for the fourth-order curl problem. J. Comput. Math., 30(6):565-578, 2012.

[19] V. A. Kondrat'ev. Boundary value problems for elliptic equations on domains with conical or angular points. Trudy Moskov. Mat. Obshch., 16:209-292, 1967. In Russian.

[20] V. Kozlov and V. Maz'ya. Spectral properties of operator pencils, generated through elliptic boundary value problems in a cone. Funkcionalniて analis $i$ ego priloshenija, 2:38-46, 1988. In Russian. 
[21] V. A. Kozlov, V. G. Maz'ya, and J. Rossmann. Elliptic boundary value problems in domains with point singularities, volume 52 of Mathematical Surveys and Monographs. American Mathematical Society, Providence, RI, 1997.

[22] V. A. Kozlov, V. G. Maz'ya, and J. Rossmann. Spectral Problems Associated with Corner Singularities of Solutions to Elliptic Equations. Mathematical Surveys and Monographs, 85. American Mathematical Society, Providence, RI, 2001.

[23] P. Monk. Finite element methods for Maxwell's equations. Numer. Math. Scientific Comp. Oxford Univ. Press, New York, 2003.

[24] P. Monk and J. Sun. Finite element methods for Maxwell's transmission eigenvalues. SIAM J. Sci. Comput., 34(3):B247-B264, 2012.

[25] S. A. Nazarov, A. Stylianou, and G. Sweers. Hinged and supported plates with corners. Z. Angew. Math. Phys., 63(5):929-960, 2012.

[26] S. Nicaise. Polygonal Interface Problems, volume 39 of Methoden und Verfahren der mathematischen Physik. Peter Lang GmbH, Europäischer Verlag der Wissenschaften, Frankfurt/M., 1993.

[27] B.-W. Schulze. Pseudo-differential operators on manifolds with singularities, volume 24 of Studies in Mathematics and its Applications. North-Holland Publishing Co., Amsterdam, 1991.

[28] J. Seif. On the Green's function for the biharmonic equation on an infinite wedge. Trans. Amer. Math. Soc., 182:241-260, 1973.

[29] J. Sun. A mixed FEM for the quad-curl eigenvalue problem. Numer. Math., 132(1):185-200, 2016.

[30] H. Walden and R. B. Kellogg. Numerical determination of the fundamental eigenvalue for the Laplace operator on a spherical domain. J. Eng. Math., 11:299-318, 1977.

[31] B. Zheng, Q. Hu, and J. Xu. A nonconforming finite element method for fourth order curl equations in $\mathbb{R}^{3}$. Math. Comp., 80(276):1871-1886, 2011. 